\title{
Taking the pulse of Earth's tropical forests using networks of highly distributed plots ${ }^{\text {is }}$
}

ForestPlots.net ${ }^{\mathrm{nx}}$, Cecilia Blundo ${ }^{\mathrm{a}}$, Julieta Carilla ${ }^{\mathrm{a}}$, Ricardo Grau ${ }^{\mathrm{a}}$, Agustina Malizia ${ }^{\mathrm{a}}$, Lucio Malizia $^{\mathrm{b}}$, Oriana Osinaga-Acosta ${ }^{\mathrm{a}}$, Michael Bird ${ }^{\mathrm{c}}$, Matt Bradford ${ }^{\mathrm{d}}$, Damien Catchpole ${ }^{\mathrm{e}}$, Andrew Ford ${ }^{\mathrm{d}}$, Andrew Graham ${ }^{\mathrm{f}}$, David Hilbert ${ }^{\mathrm{g}}$, Jeanette Kemp ${ }^{\mathrm{h}}$, Susan Laurance ${ }^{\mathrm{i}}$, William Laurance $^{\mathrm{i}}$, Francoise Yoko Ishida ${ }^{\mathrm{j}}$, Andrew Marshall ${ }^{\mathrm{k}, 1, \mathrm{~m}}$, Catherine Waite ${ }^{\mathrm{k}}$, Hannsjoerg Woell ${ }^{\mathrm{n}}$, Jean-Francois Bastin ${ }^{\mathrm{o}}$, Marijn Bauters ${ }^{\mathrm{p}}$, Hans Beeckman ${ }^{\mathrm{q}}$, Pfascal Boeckx ${ }^{\mathrm{r}}$, Jan Bogaert $^{\mathrm{s}}$, Charles De Canniere ${ }^{\mathrm{t}}$, Thales de Haulleville ${ }^{\mathrm{u}}$, Jean-Louis Doucet ${ }^{\mathrm{v}}$, Olivier Hardy ${ }^{\mathrm{w}}$, Wannes Hubau ${ }^{\mathrm{x}}$, Elizabeth Kearsley ${ }^{\mathrm{y}}$, Hans Verbeeck ${ }^{\mathrm{z}}$, Jason Vleminckx ${ }^{\text {aa }}$, Steven W. Brewer ${ }^{\mathrm{ab}}$, Alfredo Alarcón ${ }^{\mathrm{ac}}$, Alejandro Araujo-Murakami ${ }^{\text {ad }}$, Eric Arets ${ }^{\mathrm{ae}}$, Luzmila Arroyo $^{\text {ad }}$, Ezequiel Chavez ${ }^{\text {af }}$, Todd Fredericksen ${ }^{\text {ac }}$, René Guillén Villaroel ${ }^{\text {ag }}$, Gloria Gutierrez Sibauty $^{\text {ah }}$, Timothy Killeen ${ }^{\text {ai }}$, Juan Carlos Licona ${ }^{\text {ac }}$, John Lleigue ${ }^{\text {ae }}$, Casimiro Mendoza ${ }^{\text {aj }}$, Samaria Murakami ${ }^{\text {ae }}$, Alexander Parada Gutierrez ${ }^{\text {ad }}$, Guido Pardo ${ }^{\text {ak }}$, Marielos Peña-Claros ${ }^{\mathrm{ae}}$, Lourens Poorter ${ }^{\mathrm{ae}}$, Marisol Toledo ${ }^{\mathrm{al}}$, Jeanneth Villalobos Cayo ${ }^{\mathrm{am}}$, Laura Jessica Viscarra $^{\text {ai }}$, Vincent Vos ${ }^{\text {an }}$, Jorge Ahumada ${ }^{\text {ao }}$, Everton Almeida ${ }^{\text {ap }}$, Jarcilene Almeida ${ }^{\text {aq }}$, Edmar Almeida de Oliveira ${ }^{\text {ar }}$, Wesley Alves da Cruz ${ }^{\text {as }}$, Atila Alves de Oliveira ${ }^{\text {at }}$, Fabrício Alvim Carvalho ${ }^{\text {au }}$, Flávio Amorim Obermuller ${ }^{\text {av }}$, Ana Andrade ${ }^{\text {aw }}$, Fernanda Antunes Carvalho ${ }^{\text {ax }}$, Simone Aparecida Vieira ${ }^{\text {ay }}$, Ana Carla Aquino ${ }^{\mathrm{az}}$, Luiz Aragão ${ }^{\mathrm{ba}}$, Ana Claudia Araújo ${ }^{\mathrm{bb}}$, Marco Antonio Assis ${ }^{\mathrm{bc}}$, Jose Ataliba Mantelli Aboin Gomes ${ }^{\text {bd }}$, Fabrício Baccaro ${ }^{\text {be }}$, Plínio Barbosa de Camargo bf Paulo Barni ${ }^{\text {bg }}$, Jorcely Barroso ${ }^{\text {bh }}$, Luis Carlos Bernacci ${ }^{\text {bi }}$, Kauane Bordin ${ }^{\text {bj }}$, Marcelo Brilhante de Medeiros ${ }^{\text {bk }}$, Igor Broggio ${ }^{\text {bl }}$, José Luís Camargo ${ }^{\text {av }}$, Domingos Cardoso ${ }^{\text {bm }}$, Maria Antonia Carniello ${ }^{\text {as }}$, Andre Luis Casarin Rochelle ${ }^{\text {bn }}$, Carolina Castilho ${ }^{\text {bo }}$, Antonio Alberto Jorge Farias Castro ${ }^{\text {bp }}$, Wendeson Castro ${ }^{\text {bq }}$, Sabina Cerruto Ribeiro ${ }^{\text {bh }}$, Flávia Costa ${ }^{\text {br }}$, Rodrigo Costa de Oliveira ${ }^{\text {bs }}$, Italo Coutinho ${ }^{\text {bt }}$, John Cunha ${ }^{\text {bu }}$, Lola da Costa ${ }^{\text {bv }}$, Lucia da Costa Ferreira $^{\text {bw }}$, Richarlly da Costa Silva ${ }^{\text {bx }}$, Marta da Graça Zacarias Simbine ${ }^{\text {ay }}$, Vitor de Andrade Kamimura ${ }^{\text {bc }}$, Haroldo Cavalcante de Lima ${ }^{\text {by }}$, Lia de Oliveira Melo ${ }^{\text {bz }}$, Luciano de Queiroz $^{c a}$, José Romualdo de Sousa Lima ${ }^{\mathrm{cb}}$, Mário do Espírito Santo ${ }^{\mathrm{cc}}$, Tomas Domingues ${ }^{c d}$, Nayane Cristina dos Santos Prestes ${ }^{c e}$, Steffan Eduardo Silva Carneiro ${ }^{\text {cf }}$, Fernando Elias $^{\mathrm{cg}}$, Gabriel Eliseu ${ }^{\mathrm{cf}}$, Thaise Emilio ${ }^{\mathrm{ch}}$, Camila Laís Farrapo ${ }^{\text {ci }}$, Letícia Fernandes ${ }^{\text {bh }}$, Gustavo Ferreira $^{c f}$, Joice Ferreira ${ }^{\text {bk }}$, Leandro Ferreira ${ }^{\mathrm{cj}}$, Socorro Ferreira ${ }^{\mathrm{ck}}$, Marcelo Fragomeni Simon ${ }^{\text {bk }}$, Maria Aparecida Freitas ${ }^{\mathrm{cl}}$, Queila S. García ${ }^{\mathrm{cm}}$, Angelo Gilberto Manzatto ${ }^{\mathrm{cn}}$, Paulo Graça ${ }^{\mathrm{co}}$, Frederico Guilherme ${ }^{\mathrm{cf}}$, Eduardo Hase ${ }^{\mathrm{cl}}$, Niro Higuchi ${ }^{\mathrm{cp}}$, Mariana Iguatemy ${ }^{\mathrm{cq}}$, Reinaldo Imbrozio Barbosa ${ }^{\mathrm{cr}}$, Margarita Jaramillo ${ }^{\mathrm{cs}}$,

\footnotetext{
The article is attributed collectively as ForestPlots.net et al., with individual authors listed alphabetically first by country of institution and secondly by family name.

* Corresponding author.

E-mail address: o.l.phillips@leeds.ac.uk (O.L. Phillips).
} 
Carlos Joly ${ }^{\mathrm{ct}}$, Joice Klipel ${ }^{\mathrm{bj}}$, Iêda Leão do Amaral ${ }^{\mathrm{cu}}$, Carolina Levis ${ }^{\mathrm{cv}}$, Antonio S. Lima ${ }^{\mathrm{cj}}$, Maurício Lima Dan ${ }^{\mathrm{cw}}$, Aline Lopes ${ }^{\mathrm{cx}}$, Herison Madeiros ${ }^{\mathrm{cy}}$, William E. Magnusson ${ }^{\mathrm{br}}$, Rubens Manoel dos Santos ${ }^{\text {ci }}$, Beatriz Marimon ${ }^{\text {ar }}$, Ben Hur Marimon Junior ${ }^{\text {ar }}$, Roberta Marotti Martelletti Grillo ${ }^{\mathrm{cz}}$, Luiz Martinelli ${ }^{\mathrm{bf}}$, Simone Matias Reis ${ }^{\mathrm{ar}}$, Salomão Medeiros da, Milton Meira-Junior ${ }^{\mathrm{db}}$, Thiago Metzker ${ }^{\mathrm{dc}}$, Paulo Morandi ${ }^{\mathrm{dd}}$, Natanael Moreira do Nascimento ${ }^{\text {cf }}$, Magna Moura ${ }^{\text {bk }}$, Sandra Cristina Müller ${ }^{\text {bj }}$, Laszlo Nagy ${ }^{\text {de }}$, Henrique Nascimento $^{\mathrm{cl}}$, Marcelo Nascimento ${ }^{\mathrm{df}}$, Adriano Nogueira Lima ${ }^{\mathrm{dg}}$, Raimunda Oliveira de Araújo ${ }^{\mathrm{cl}}$, Jhonathan Oliveira Silva ${ }^{\mathrm{dh}}$, Marcelo Pansonato di, Gabriel Pavan Sabino ${ }^{\text {bc }}$, Karla Maria Pedra de Abreu ${ }^{\text {dj }}$, Pablo José Francisco Pena Rodrigues ${ }^{\text {by }}$, Maria Piedade ${ }^{\mathrm{dk}}$, Domingos Rodrigues ${ }^{\mathrm{dl}}$, José Roberto Rodrigues Pinto ${ }^{\mathrm{db}}$, Carlos Quesada ${ }^{\mathrm{cl}}$, Eliana Ramos ${ }^{\mathrm{dm}}$, Rafael Ramos ${ }^{\mathrm{ay}}$, Priscyla Rodrigues ${ }^{\mathrm{dh}}$, Thaiane Rodrigues de Sousa ${ }^{\mathrm{dn}}$, Rafael Salomão do , Flávia Santana ${ }^{\mathrm{cl}}$, Marcos Scaranello ${ }^{\text {bn }}$, Rodrigo Scarton Bergamin ${ }^{\mathrm{bj}}$, Juliana Schietti $^{\mathrm{dp}}$, Jochen Schöngart ${ }^{\mathrm{dq}}$, Gustavo Schwartz ${ }^{\mathrm{dr}}$, Natalino Silva ${ }^{\mathrm{ds}}$, Marcos Silveira ${ }^{\mathrm{dt}}$, Cristiana Simão Seixas ${ }^{\mathrm{ay}}$, Marta Simbine ${ }^{\mathrm{bn}}$, Ana Claudia Souza ${ }^{\mathrm{bc}}$, Priscila Souza $^{\text {br }}$, Rodolfo Souza ${ }^{\text {du }}$, Tereza Sposito ${ }^{\text {dc }}$, Edson Stefani Junior ${ }^{\text {bn }}$, Julio Daniel do Vale ${ }^{\mathrm{dv}}$, Ima Célia Guimarães Vieira ${ }^{\mathrm{dw}}$, Dora Villela ${ }^{\mathrm{df}}$, Marcos Vital ${ }^{\mathrm{bb}}$, Haron Xaud $^{\text {bo }}$, Katia Zanini ${ }^{\text {bj }}$, Charles Eugene Zartman ${ }^{c o}$, Nur Khalish Hafizhah Ideris ${ }^{\mathrm{dx}}$, Faizah binti Hj Metali ${ }^{\text {dy }}$, Kamariah Abu Salim ${ }^{\text {dy }}$, Muhd Shahruney Saparudin ${ }^{\mathrm{dx}}$, Rafizah Mat Serudin ${ }^{\mathrm{dx}}$, Rahayu Sukmaria Sukri ${ }^{\mathrm{dz}}$, Serge Begne ${ }^{\mathrm{ea}}$, George Chuyong ${ }^{\mathrm{eb}}$, Marie Noel Djuikouo ${ }^{\text {ec }}$, Christelle Gonmadje ${ }^{\text {ed }}$, Murielle Simo-Droissart ${ }^{\text {ee }}$, Bonaventure Sonké $^{\mathrm{ee}}$, Hermann Taedoumg ${ }^{\text {ef,eg }}$, Lise Zemagho ${ }^{\mathrm{ee}}$, Sean Thomas ${ }^{\text {eh }}$, Fidèle Baya ${ }^{\text {ei }}$, Gustavo Saiz $^{\mathrm{ej}}$, Javier Silva Espejo ${ }^{\mathrm{ek}}$, Dexiang Chen ${ }^{\mathrm{el}}$, Alan Hamilton ${ }^{\mathrm{em}}$, Yide $\mathrm{Li}^{\mathrm{el}}$, Tushou Luo ${ }^{\mathrm{el}}$, Shukui Niu ${ }^{\mathrm{en}}$, Han Xu ${ }^{\mathrm{el}}$, Zhang Zhou ${ }^{\mathrm{el}}$, Esteban Álvarez-Dávila ${ }^{\mathrm{e}}$, Juan Carlos Andrés Escobar ${ }^{\text {ep }}$, Henry Arellano-Peña ${ }^{\text {eq, }}$, Jaime Cabezas Duarte ${ }^{\text {er }}$, Jhon Calderón ${ }^{\mathrm{es}}$, Lina Maria Corrales Bravo ${ }^{\mathrm{er}}$, Borish Cuadrado ${ }^{\mathrm{et}}$, Hermes Cuadros ${ }^{\mathrm{eu}}$, Alvaro Duque $^{\mathrm{ev}}$, Luisa Fernanda Duque ${ }^{\mathrm{ew}}$, Sandra Milena Espinosa ${ }^{\mathrm{ep}}$, Rebeca Franke-Ante ${ }^{\mathrm{ex}}$, Hernando García $^{\mathrm{ey}}$, Alejandro Gómez ${ }^{\mathrm{ez}}$, Roy González-M. ${ }^{\text {fa }}$, Álvaro Idárraga-Piedrahíta ${ }^{\mathrm{fb}}$, Eliana Jimenez ${ }^{\mathrm{fc}}$, Rubén Jurado ${ }^{\mathrm{es}}$, Wilmar López Oviedo ${ }^{\mathrm{fd}}$, René López-Camacho ${ }^{\mathrm{fe}}$, Omar Aurelio Melo Cruz ${ }^{\mathrm{ff}}$, Irina Mendoza Polo ${ }^{\mathrm{ew}}$, Edwin Paky ${ }^{\mathrm{ep}}$, Karen Pérez ${ }^{\mathrm{fg}}$, Angel Pijachi ${ }^{\mathrm{ep}}$, Camila Pizano ${ }^{\mathrm{fh}}$, Adriana Prieto ${ }^{\mathrm{fi}}$, Laura Ramos ${ }^{\mathrm{fj}}$, Zorayda Restrepo Correa ${ }^{\mathrm{fk}}$, James Richardson $^{\mathrm{fl}}$, Elkin Rodríguez ${ }^{\mathrm{et}}$, Gina M. Rodriguez M. ${ }^{\mathrm{fm}}$, Agustín Rudas ${ }^{\mathrm{fi}}$, Pablo Stevenson $^{\mathrm{fn}}$, Markéta Chudomelová ${ }^{\mathrm{fo}}$, Martin Dancak ${ }^{\mathrm{fp}}$, Radim Hédl ${ }^{\mathrm{fo}}$, Stanislav Lhota ${ }^{\mathrm{fq}}$, Martin Svatek $^{\mathrm{fr}}$, Jacques Mukinzi ${ }^{\mathrm{fs}}$, Corneille Ewango ${ }^{\mathrm{ft}}$, Terese Hart ${ }^{\mathrm{fu}}$, Emmanuel Kasongo Yakusu $^{\mathrm{fv}}$, Janvier Lisingo ${ }^{\mathrm{fw}}$, Jean-Remy Makana ${ }^{\mathrm{ft}}$, Faustin Mbayu ${ }^{\mathrm{fx}}$, Benjamin Toirambe ${ }^{\mathrm{fy}}$, John Tshibamba Mukendi ${ }^{\mathrm{fx}}$, Lars Kvist ${ }^{\mathrm{fz}}$, Gustav Nebel ${ }^{\text {ga }}$, Selene Báez ${ }^{\mathrm{gb}}$, Carlos Céron $^{g c}$, Daniel M. Griffith ${ }^{g d}$, Juan Ernesto Guevara Andino ${ }^{g e, g f}$, David Neill ${ }^{g g}$, Walter Palacios ${ }^{\text {gh }}$, Maria Cristina Peñuela-Mora ${ }^{\text {gi }}$, Gonzalo Rivas-Torres ${ }^{\text {gj, gk, gl,gm,gn }}$, Gorky Villa $^{\mathrm{go}}$, Sheleme Demissie ${ }^{\mathrm{gp}}$, Tadesse Gole ${ }^{\mathrm{gq}}$, Techane Gonfa ${ }^{\mathrm{gr}}$, Kalle Ruokolainen ${ }^{\mathrm{gs}}$, Michel Baisie ${ }^{\mathrm{gt}}$, Fabrice Bénédet ${ }^{\mathrm{gt}}$, Wemo Betian ${ }^{\mathrm{gu}}$, Vincent Bezard ${ }^{\mathrm{gv}}$, Damien Bonal ${ }^{\mathrm{gw}}$, Jerôme Chave $^{\text {gx }}$, Vincent Droissart ${ }^{\text {gy }}$, Sylvie Gourlet-Fleury ${ }^{\mathrm{gz}}$, Annette Hladik ${ }^{\text {ha }}$, Nicolas Labrière ${ }^{\mathrm{gx}}$, Pétrus Naisso ${ }^{\mathrm{gt}}$, Maxime Réjou-Méchain ${ }^{\mathrm{gy}}$, Plinio Sist ${ }^{\mathrm{gt}}$, Lilian Blanc ${ }^{\mathrm{nt}}$, Benoit Burban $^{\mathrm{hb}}$, Géraldine Derroire ${ }^{\mathrm{hc}}$, Aurélie Dourdain ${ }^{\mathrm{hc}}$, Clement Stahl ${ }^{\mathrm{nu}}$, Natacha Nssi Bengone $^{\text {hd }}$, Eric Chezeaux ${ }^{\text {he }}$, Fidèle Evouna Ondo ${ }^{\text {hf }}$, Vincent Medjibe ${ }^{\text {hg }}$, Vianet Mihindou $^{\text {hh }}$, Lee White ${ }^{\text {hi }}$, Heike Culmsee ${ }^{\text {hj }}$, Cristabel Durán Rangel ${ }^{\text {hk }}$, Viviana Horna ${ }^{\text {hl }}$, Florian Wittmann $^{\text {hm }}$, Stephen Adu-Bredu ${ }^{\text {hn }}$, Kofi Affum-Baffoe ${ }^{\text {ho }}$, Ernest Foli ${ }^{\text {hn }}$, Michael Balinga $^{\text {hp }}$, Anand Roopsind ${ }^{\text {hq }}$, James Singh ${ }^{\text {hr }}$, Raquel Thomas ${ }^{\text {hq }}$, Roderick Zagt ${ }^{\text {hs }}$, Indu K. Murthy ${ }^{\text {ht }}$, Kuswata Kartawinata ${ }^{\text {hu,hv }}$, Edi Mirmanto ${ }^{\text {hw }}$, Hari Priyadi ${ }^{\text {hu }}$, Ismayadi Samsoedin $^{\text {hx }}$, Terry Sunderland ${ }^{\mathrm{nv}}$, Ishak Yassir ${ }^{\text {hy }}$, Francesco Rovero ${ }^{\mathrm{hz}}$, Barbara Vinceti $^{\text {ia }}$, Bruno Hérault ${ }^{\text {ib }}$, Shin-Ichiro Aiba ${ }^{\text {ic }}$, Kanehiro Kitayama ${ }^{\text {id }}$, Armandu Daniels ${ }^{\text {ie }}$, Darlington Tuagben ${ }^{\text {ie }}$, John T. Woods ${ }^{\text {if }}$, Muhammad Fitriadi ${ }^{\text {ig }}$, Alexander Karolus $^{\text {ih }}$, Kho Lip Khoon ${ }^{\text {ii }}$, Noreen Majalap ${ }^{\text {ij }}$, Colin Maycock ${ }^{\text {ik }}$, Reuben Nilus ${ }^{\text {il }}$, Sylvester Tan ${ }^{\text {im }}$, Almeida Sitoe ${ }^{\text {in }}$, Indiana Coronado G. ${ }^{\text {io }}$, Lucas Ojo ${ }^{\text {ip }}$, Rafael de Assis ${ }^{\text {iq }}$, Axel Dalberg Poulsen ${ }^{\text {ir }}$, Douglas Sheil ${ }^{\text {is }}$, Karen Arévalo Pezo ${ }^{\text {it }}$, Hans Buttgenbach Verde ${ }^{\text {ny }}$, 
Victor Chama Moscoso ${ }^{\text {iv }}$, Jimmy Cesar Cordova Oroche ${ }^{\text {it }}$, Fernando Cornejo Valverde ${ }^{\text {iw }}$, Massiel Corrales Medina ${ }^{\text {ix }}$, Nallaret Davila Cardozo ${ }^{\text {iy }}$, Jano de Rutte Corzo ${ }^{\text {iz }}$, Jhon del Aguila Pasquel ${ }^{\mathrm{ja}}$, Gerardo Flores Llampazo ${ }^{\mathrm{jb}}$, Luis Freitas ${ }^{\mathrm{ja}}$, Darcy Galiano Cabrera ${ }^{\mathrm{jc}}$, Roosevelt García Villacorta ${ }^{i t}$, Karina Garcia Cabrera ${ }^{\text {jc }}$, Diego García Soria ${ }^{\text {ja }}$, Leticia Gatica Saboya ${ }^{\text {it }}$, Julio Miguel Grandez Rios ${ }^{\text {it }}$, Gabriel Hidalgo Pizango ${ }^{\text {ja }}$, Eurídice Honorio Coronado $^{\text {ja }}$, Isau Huamantupa-Chuquimaco ${ }^{\mathrm{jc}}$, Walter Huaraca Huasco ${ }^{\mathrm{jc}}$, Yuri Tomas Huillca Aedo ${ }^{\mathrm{jc}}$, Jose Luis Marcelo Peña ${ }^{\mathrm{iu}}$, Abel Monteagudo Mendoza ${ }^{\mathrm{jc}}$, Vanesa Moreano Rodriguez ${ }^{\text {ny }}$, Percy Núñez Vargas ${ }^{\text {jc }}$, Sonia Cesarina Palacios Ramos ${ }^{\text {ny }}$, Nadir Pallqui Camacho ${ }^{\text {jc }}$, Antonio Peña Cruz ${ }^{\text {iv }}$, Freddy Ramirez Arevalo ${ }^{\text {it }}$, José Reyna Huaymacari ${ }^{\text {it }}$, Carlos Reynel Rodriguez ${ }^{\text {ny }}$, Marcos Antonio Ríos Paredes it, Lily Rodriguez Bayona ${ }^{\text {jd }}$, Rocio del Pilar Rojas Gonzales ${ }^{\text {iv }}$, Maria Elena Rojas Peña ${ }^{\text {it }}$, Norma Salinas Revilla $^{\text {je }}$, Yahn Carlos Soto Shareva ${ }^{\text {iv }}$, Raul Tupayachi Trujillo ${ }^{\text {jf }}$, Luis Valenzuela Gamarra ${ }^{\text {iv }}$, Rodolfo Vasquez Martinez ${ }^{\text {iv }}$, Jim Vega Arenas ${ }^{\text {it }}$, Christian Amani ${ }^{\text {jg }}$, Suspense Averti Ifo ${ }^{\text {jh }}$, Yannick Bocko ${ }^{\text {jh }}$, Patrick Boundja ${ }^{j i}$, Romeo Ekoungoulou ${ }^{j j}$, Mireille Hockemba $^{\mathrm{ji}}$, Donatien Nzala ${ }^{\mathrm{jk}}$, Alusine Fofanah ${ }^{\mathrm{jl}}$, David Taylor ${ }^{\mathrm{jm}}$, Guillermo Bañares-de Dios $^{\text {jn }}$, Luis Cayuela $^{\text {jn }}$, Íñigo Granzow-de la Cerda $^{\text {jo }}$, Manuel Macía $^{\text {jp }}$, Juliana Stropp $^{\mathrm{jq}}$, Maureen Playfair $^{\mathrm{jr}}$, Verginia Wortel ${ }^{\mathrm{jr}}$, Toby Gardner ${ }^{\mathrm{js}}$, Robert Muscarella ${ }^{\mathrm{jt}}$, Hari Priyadi $^{\text {ju }}$, Ervan Rutishauser ${ }^{\mathrm{jv}}$, Kuo-Jung Chao ${ }^{\mathrm{jw}}$, Pantaleo Munishi ${ }^{\mathrm{jx}}$, Olaf Bánki ${ }^{\mathrm{jy}}$, Frans Bongers $^{\mathrm{jz}}$, Rene Boot ${ }^{\mathrm{ka}}$, Gabriella Fredriksson ${ }^{\mathrm{kb}}$, Jan Reitsma ${ }^{\mathrm{kc}}$, Hans ter Steege ${ }^{\mathrm{jy}}$, Tinde van Andel ${ }^{\mathrm{jy}}$, Peter van de Meer ${ }^{\mathrm{kd}}$, Peter van der Hout ${ }^{\mathrm{ke}}$, Mark van Nieuwstadt ${ }^{\mathrm{kf}}$, Bert van Ulft ${ }^{\mathrm{kg}}$, Elmar Veenendaal ${ }^{\mathrm{kh}}$, Ronald Vernimmen ${ }^{\mathrm{ki}}$, Pieter Zuidema ${ }^{\mathrm{kh}}$, Joeri Zwerts ${ }^{\mathrm{kj}}$, Perpetra Akite ${ }^{\mathrm{kk}}$, Robert Bitariho ${ }^{\mathrm{kl}}$, Colin Chapman ${ }^{\mathrm{km}}$, Eilu Gerald ${ }^{\mathrm{ko}}$, Miguel Leal ${ }^{\mathrm{kp}}$, Patrick Mucunguzi ${ }^{\mathrm{kn}}$, Katharine Abernethy ${ }^{\mathrm{kq}}$, Miguel Alexiades ${ }^{\mathrm{kr}}$, Timothy R. Baker ${ }^{\mathrm{ks}}$, Karina Banda ${ }^{\mathrm{ks}}$, Lindsay Banin ${ }^{\mathrm{kt}}$, Jos Barlow ${ }^{\mathrm{ku}}$, Amy Bennett ${ }^{\mathrm{ks}}$, Erika Berenguer ${ }^{\mathrm{ku}}$, Nicholas Berry ${ }^{\mathrm{kw}}$, Neil M. Bird ${ }^{\mathrm{kx}}$, George A. Blackburn ${ }^{\mathrm{ku}}$, Francis Brearley ${ }^{\mathrm{ky}}$, Roel Brienen ${ }^{\mathrm{ks}}$, David Burslem $^{\mathrm{kz}}$, Lidiany Carvalho ${ }^{\mathrm{la}}$, Percival Cho ${ }^{\mathrm{ku}}$, Fernanda Coelho ${ }^{\mathrm{ks}}$, Murray Collins ${ }^{\mathrm{lb}}$, David Coomes $^{\text {lc }}$, Aida Cuni-Sanchez ${ }^{\text {ld }}$, Greta Dargie ${ }^{\text {ks }}$, Kyle Dexter ${ }^{\text {lb }}$, Mat Disney ${ }^{\text {le }}$, Freddie Draper $^{\mathrm{ks}}$, Muying Duan ${ }^{\mathrm{lf}}$, Adriane Esquivel-Muelbert ${ }^{\mathrm{lg}}$, Robert Ewers ${ }^{\text {lf }}$, Belen Fadrique $^{\mathrm{ks}}$, Sophie Fauset ${ }^{\mathrm{lh}}$, Ted R. Feldpausch ${ }^{\mathrm{li}}$, Filipe França ${ }^{\mathrm{lj}}$, David Galbraith ${ }^{\mathrm{ks}}$, Martin Gilpin $^{\mathrm{ks}}$, Emanuel Gloor ${ }^{\mathrm{ks}}$, John Grace ${ }^{\mathrm{k}}$, Keith Hamer ${ }^{11}$, David Harris ${ }^{1 \mathrm{~m}}$, Kath Jeffery ${ }^{\mathrm{ln}}$, Tommaso Jucker $^{1 \mathrm{o}}$, Michelle Kalamandeen ${ }^{\mathrm{ks}, \mathrm{lp}, \mathrm{lq}}$, Bente Klitgaard ${ }^{\mathrm{lr}}$, Aurora Levesley ${ }^{\mathrm{ks}}$, Simon L. Lewis ${ }^{k s}$, Jeremy Lindsell ${ }^{1 s}$, Gabriela Lopez-Gonzalez ${ }^{\mathrm{ks}}$, Jon Lovett ${ }^{\mathrm{ks}}$, Yadvinder Malhi $^{\text {lt }}$, Toby Marthews ${ }^{\text {lu }}$, Emma McIntosh ${ }^{\text {lv }}$, Karina Melgaço ${ }^{\mathrm{ks}}$, William Milliken ${ }^{\mathrm{lw}}$, Edward Mitchard $^{\mathrm{lb}}$, Peter Moonlight ${ }^{\mathrm{lm}}$, Sam Moore ${ }^{\mathrm{lv}}$, Alexandra Morel ${ }^{\mathrm{lx}}$, Julie Peacock ${ }^{\mathrm{ks}}$, Kelvin S.-H. Peh ${ }^{1 y}$, Colin Pendry ${ }^{\mathrm{lm}}$, R. Toby Pennington ${ }^{\mathrm{la}, \mathrm{lm}}$, Luciana de Oliveira Pereira ${ }^{\text {la }}$, Carlos Peres $^{\mathrm{lz}}$, Oliver L. Phillips ${ }^{\mathrm{ks}, *}$, Georgia Pickavance ${ }^{\mathrm{ks}}$, Thomas Pugh ${ }^{\mathrm{lg}}$, Lan Qie ${ }^{\mathrm{nw}}$, Terhi Riutta $^{\mathrm{kv}}$, Katherine Roucoux ${ }^{\mathrm{ma}}$, Casey Ryan ${ }^{\mathrm{lk}}$, Tiina Sarkinen ${ }^{\mathrm{lm}}$, Camila Silva Valeria ${ }^{\mathrm{ku}}$, Dominick Spracklen $^{\mathrm{mb}}$, Suzanne Stas ${ }^{\mathrm{mb}}$, Martin Sullivan ${ }^{\mathrm{ks}}$, Michael Swaine ${ }^{\mathrm{mc}}$, Joey Talbot $^{\text {ks, md }}{ }^{\text {, James Taplin }}{ }^{\mathrm{me}}$, Geertje van der Heijden ${ }^{\mathrm{mf}}$, Laura Vedovato ${ }^{\text {la }}$, Simon Willcock ${ }^{\mathrm{mg}}$, Mathew Williams ${ }^{\mathrm{lk}}$, Luciana Alves ${ }^{\mathrm{mh}}$, Patricia Alvarez Loayza ${ }^{\mathrm{mi}}$, Gabriel Arellano $^{\mathrm{mj}}$, Cheryl Asa ${ }^{\mathrm{mk}}$, Peter Ashton ${ }^{\mathrm{ml}}$, Gregory Asner ${ }^{\mathrm{mm}}$, Terry Brncic ${ }^{\mathrm{mn}}$, Foster Brown $^{\mathrm{mo}}$, Robyn Burnham ${ }^{\mathrm{mp}}$, Connie Clark ${ }^{\mathrm{mq}}$, James Comiskey ${ }^{\mathrm{mr}}$, Gabriel Damasco ${ }^{\mathrm{ms}}$, Stuart Davies $^{\mathrm{mt}}$, Tony Di Fiore ${ }^{\mathrm{mu}}$, Terry Erwin ${ }^{\mathrm{mv}}$, William Farfan-Rios ${ }^{\mathrm{mw}}$, Jefferson Hall ${ }^{\mathrm{mx}}$, David Kenfack $^{\mathrm{my}}$, Thomas Lovejoy ${ }^{\mathrm{mz}}$, Roberta Martin ${ }^{\mathrm{mn}}$, Olga Martha Montiel ${ }^{\text {na }}$, John Pipoly ${ }^{\text {nb, nc }}$, Nigel Pitman ${ }^{\text {nd }}$, John Poulsen ${ }^{\text {mq }}$, Richard Primack ${ }^{\text {ne }}$, Miles Silman ${ }^{\text {nf }}$, Marc Steininger $^{\mathrm{ng}}$, Varun Swamy ${ }^{\mathrm{nh}}$, John Terborgh ${ }^{\mathrm{mi}}$, Duncan Thomas ${ }^{\mathrm{ni}}$, Peter Umunay ${ }^{\mathrm{nj}}$, Maria Uriarte $^{\mathrm{nk}}$, Emilio Vilanova Torre ${ }^{\mathrm{nl}}$, Ophelia Wang ${ }^{\mathrm{nm}}$, Kenneth Young ${ }^{\mathrm{nn}}$, Gerardo A. Aymard C. ${ }^{\text {no }}$, Lionel Hernández ${ }^{\text {np }}$, Rafael Herrera Fernández ${ }^{\text {nq }}$, Hirma Ramírez-Angulo $^{\mathrm{nr}}$, Pedro Salcedo ${ }^{\mathrm{nr}}$, Elio Sanoja ${ }^{\mathrm{np}}$, Julio Serrano ${ }^{\mathrm{nr}}$, Armando Torres-Lezama ${ }^{\mathrm{nr}}$, Tinh Cong Le ${ }^{\mathrm{ns}}$, Trai Trong Le ${ }^{\mathrm{ns}}$, Hieu Dang Tran ${ }^{\mathrm{ns}}$

\footnotetext{
a Instituto de Ecología Regional (IER), Universidad Nacional de Tucumán (UNT), Consejo Nacional de Investigaciones Científicas y Técnicas (CONICET), Argentina

${ }^{\mathrm{b}}$ Facultad de Ciencias Agrarias, Universidad Nacional de Jujuy, Jujuy, Argentina

c James Cook University (JCU), Australia

${ }^{\mathrm{d}}$ CSIRO (Commonwealth Scientific and Industrial Research Organisation), Australia
} 
e School of Land \& Food, University of Tasmania, Australia

${ }^{\mathrm{f}}$ CSIRO Tropical Forest Research Centre, Australia

${ }^{\mathrm{g}}$ Independent Researcher, Australia

${ }^{\mathrm{h}}$ Environmental Protection Agency (EPA), Australia

${ }^{\mathrm{i}}$ Centre for Tropical Environmental and Sustainability Science (TESS), College of Marine and Environmental Sciences, James Cook University, Australia

${ }^{\mathrm{j}}$ Centre for Tropical Environmental and Sustainability Science, College of Science and Engineering, James Cook University, Australia

${ }^{\mathrm{k}}$ University of the Sunshine Coast, Australia

${ }^{1}$ University of York, United Kingdom

${ }^{\mathrm{m}}$ Flamingo Land Ltd., United Kingdom

n Sommersbergseestrasse, Austria

${ }^{\circ}$ Ghent University, Belgium

${ }^{\mathrm{p}}$ CAVElab, Ghent University, Belgium

${ }^{\mathrm{q}}$ Royal Museum for Central Africa - Service of Wood Biology, Belgium

${ }^{\mathrm{r}}$ Isotope Bioscience Laboratory-ISOFYS, Ghent University, Belgium

${ }^{s}$ Gembloux Agro-Bio Tech, Université de Liege, Belgium

${ }^{\mathrm{t}}$ Landscape Ecology and Vegetal Production Systems Unit, Belgium

" CAVElab Computational \& Applied Vegetation Ecology, Ghent University, Belgium

${ }^{v}$ Tropical Forestry, Forest Resources Management, Gembloux Agro-Bio Tech, University of Liege, Belgium

${ }^{\mathrm{w}}$ Université Libre de Bruxelles (ULB), Evolutionary Biology and Ecology, Belgium

${ }^{\mathrm{x}}$ Royal Museum for Central Africa, Belgium

${ }^{y}$ Royal Museum for Central Africa, Ghent University, Belgium

${ }^{\mathrm{z}}$ Department of Environment, Ghent University, Belgium

${ }^{\text {aa }}$ Service Evolution Biologique et Ecologie, Belgium

${ }^{\mathrm{ab}}$ Belize Foundation for Research and Environmental Education, Belize

${ }^{\text {ac }}$ IBIF, Bolivia

${ }^{\text {ad }}$ Museo de Historia Natural Noel Kempff Mercado, Universidad Autonoma Gabriel Rene Moreno, Bolivia

ae PROMAB, Bolivia

${ }^{\text {af }}$ Museo Noel Kempff, Bolivia

ag Consultor Independiente, Bolivia

ah Jardin Botanico Municipal de Santa Cruz, Bolivia

${ }^{\text {ai }}$ Museo de Historia Natural Noel Kempff Mercado, Bolivia

${ }^{\text {aj }}$ Forest Management in Bolivia, Bolivia

${ }^{\text {ak }}$ Universidad Autónoma del Beni Riberalta, Bolivia

${ }^{\text {al }}$ Museo de Historia Natural Noel Kempff, Bolivia

${ }^{\text {am }}$ Herbario del Sur de Bolivia, Bolivia

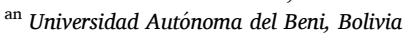

ao Conservation International, Brazil

ap Instituto de Biodiversidade e Floresta, Universidade Federal do Oeste do Pará, Brazil

${ }^{\text {aq }}$ Universidade Federal de Pernambuco, Brazil

${ }^{\mathrm{ar}}$ Universidade do Estado de Mato Grosso, Brazil

as Universidade do Estado de Mato Grosso (UNEMAT), Brazil

at Projeto TEAM - Manaus, Brazil

au Universidade Federal de Juiz de Fora (UFJF), Brazil

${ }^{\text {av }}$ Universidade Federal do Rio de Janeiro, Brazil

${ }^{\text {aw }}$ Instituto Nacional de Pesquisas da Amazônia, Projeto Dinâmica Biológica de Fragmentos Florestais, Brazil

${ }^{\text {ax }}$ Departamento de Genética, Ecologia e Evolução, Universidade Federal de Minas Gerais, Brazil

ay Universidade Estadual de Campinas, Brazil

${ }^{a z}$ Laboratório de Ecologia de Comunidades e Funcionamento de Ecossistemas-ECoFERP, Departamento de Biologia, Faculdade de Filosofia, Ciências e Letras, USP, Ribeirão Preto, SP, Brazil

ba National Institute for Space Research (INPE), Brazil

${ }^{\mathrm{bb}}$ Universidade Federal de Roraima (UFRR), Brazil

bc UNESP - São Paulo State University, Brazil

bd Carbonozero Consultoria Ambiental, Brazil

be Departamento de Biologia, Universidade Federal do Amazonas (UFAM), Brazil

${ }^{b f}$ Centro de Energia Nuclear na Agricultura, Universidade de São Paulo, Brazil

bg UERR - Campus Rorainópolis, Brazil

${ }^{\text {bh }}$ Universidade Federal do Acre, Brazil

bi Instituto Agronômico de Campinas, Brazil

bj Universidade Federal do Rio Grande do Sul, Brazil

${ }^{\mathrm{bk}}$ Embrapa, Brazil

${ }^{\text {bl }}$ Universidade Estadual do Norte Fluminense (UENF), Brazil

${ }^{\mathrm{bm}}$ Universidade Federal da Bahia (UFBA), Brazil

bn Instituto de Biologia, Universidade Estadual de Campinas, Brazil

bo Embrapa, Roraima, Brazil

bp Universidade Federal do Piauí (UFPI), Teresina, Brazil

${ }^{\mathrm{bq}}$ Botany and Plant Ecology Laboratory, Federal University of Acre, Brazil

br INPA- Instituto Nacional de Pesquisas da Amazônia, Brazil

bs UERR - Campus Boa Vista, Brazil

bt Universidade Federal do Ceará, Brazil

${ }^{b u}$ Universidade Federal de Campina Grande, Brazil

bv Universidade Federal do Para, Brazil

${ }^{\mathrm{bw}}$ Núcleo de Estudos e Pesquisas Ambientais, Universidade Estadual de Campinas, Brazil

${ }^{b x}$ Instituto Federal de Educação, Ciência e Tecnologia do Acre, Brazil

by Instituto de Pesquisas Jardim Botânico do Rio de Janeiro, Brazil

${ }^{\mathrm{bz}}$ Universidade Federal do Oeste do Pará, Brazil

${ }^{\text {ca } U E F S, ~ D e p t o . ~ d e ~ C i e ̂ n c i a s ~ B i o l o ́ g i c a s, ~ B r a z i l ~}$

${ }^{\mathrm{cb}}$ Universidade Federal do Agreste de Pernambuco (UFAPE), Brazil

${ }^{\mathrm{cc}}$ Universidade Estadual de Montes Claros, Brazil

cd FFCLRP-USP/Br, Brazil

ce UNEMAT, Brazil 
${ }^{\text {cf }}$ Universidade Federal de Jataí, Brazil

${ }^{\mathrm{cg}}$ Universidade Federal do Pará, Instituto de Ciências Biológicas, Brazil

ch Universidade de Campinas, Brazil

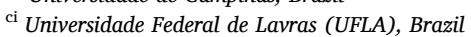

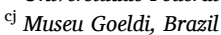

${ }^{\mathrm{ck}}$ Embrapa Amazônia Oriental, Brazil

${ }^{\mathrm{cl}}$ Instituto Nacional de Pesquisas da Amazônia, Brazil

${ }^{\mathrm{cm}}$ UFMG - Universidade Federal de Minas Gerais, Brazil

${ }^{\mathrm{cn}}$ Fundação Universidade Fedral de Rondônia - UNIR, Brazil

${ }^{c o}$ INPA- Instituto Nacional de Pesquisas Amazônicas, Brazil

cp Instituto Nacional de Pesquisas da Amazônia - Coordenação de Pesquisas em Silvicultura Tropical, Brazil

cq Jardim Botânico do Rio de Janeiro, Brazil

${ }^{\mathrm{cr}}$ National Institute for Research in Amazonia, Brazil

${ }^{\mathrm{cs}}$ Universidade Federal de Roraima (UFRR/PRONAT), Brazil

${ }^{c t}$ Universidade Estadual de Campinas/UNICAMP, Brazil

${ }^{\mathrm{cu}}$ Instituto Nacional de Pesquisas da Amazônia/CPBO, Brazil

${ }^{\mathrm{cv}}$ Universidade Federal de Santa Catarina (UFSC), Brazil

${ }^{\mathrm{cw}}$ INCAPER-Instituto Capixaba de Pesquisa, Assistência Técnica e Extensão Rural, Brazil

${ }^{\mathrm{cx}}$ INPE- Instituto Nacional de Pesquisas Espaciais, Brazil

${ }^{\text {cy }}$ Universidade de São Paulo, Brazil

${ }^{\mathrm{cz}}$ Instituto de Biociências, Universidade Estadual Paulista, Brazil

da Semiarid National Institute (INSA), Brazil

${ }^{\mathrm{db}}$ Universidade de Brasília, Departamento de Engenharia Florestal, Brazil

${ }^{\mathrm{dc}}$ IBAM - Instituto Bem Ambiental, Brazil

${ }^{\mathrm{dd}}$ Universidade do Estado de Mato Grosso, Campus de Nova Xavantina, Brazil

${ }^{\text {de }}$ University in Campinas, Brazil

${ }^{d f}$ Universidade Estadual do Norte Fluminense Darcy Ribeiro (UENF), Brazil

${ }^{\mathrm{dg}}$ LMF, Instituto Nacional de Pesquisas da Amazônia, Brazil

${ }^{\text {dh }}$ Universidade Federal do Vale do São Francisco, Brazil

di USP - University of São Paulo, Brazil

dj Instituto Federal do Espírito Santo (IFES), Brazil

${ }^{\mathrm{dk}}$ INPA - Instituto Nacional de Pesquisas da Amazônia, Grupo MAUA, Brazil

${ }^{\mathrm{d} l}$ Universidade Federal de Mato Grosso, Instituto de Ciências Naturais, Humanas e Sociais, Sinop, Brazil

dm Instituto Nacional da Mata Atlântica, Brazil

dn RAINFOR-PPBIO, Brazil

do Universidade Federal Rural da Amazônia - UFRA/CAPES, Brazil

dp Universidade Federal do Amazonas (UFAM), Brazil

dq INPA/Max-Planck Project, Brazil

${ }^{\mathrm{dr}}$ EMBRAPA- Empresa Brasileira de Pesquisa Agropecuária (Amazônia Oriental), Brazil

ds Serviço Florestal Brasileiro, Brazil

${ }^{\mathrm{dt}}$ Museu Universitário, Universidade Federal do Acre, Brazil

${ }^{\mathrm{du}}$ Universidade Federal Rural de Pernambuco, Brazil

${ }^{\mathrm{dv}}$ PUCPR - Pontifícia Universidade Católica do Paraná, Brazil

${ }^{\mathrm{dw}}$ Museu Paraense Emilio Goeldi, Brazil

${ }^{\mathrm{dx}}$ Universiti Brunei Darussalam, Brunei

dy Environmental and Life Sciences, Faculty of Science, Universiti Brunei Darussalam, Brunei

$\mathrm{dz}$ Institute for Biodiversity and Environmental Research, Universiti Brunei Darussalam, Brunei

ea Plant Systematic and Ecology Laboratory, Department of Biology, Higher Teachers' Training College, University of Yaounde I, Cameroon

${ }^{\mathrm{eb}}$ Faculty of Science, Department of Botany and Plant Physiology, University of Buea, Buea, Cameroon

${ }^{\text {ec }}$ Faculty of Science, Department of Plant Science, University of Buea, Cameroon

ed National Herbarium, Yaounde, Cameroon

ee Plant Systematics and Ecology Laboratory, Higher Teachers' Training College, University of Yaoundé I, Cameroon

ef Department of Plant Biology, Faculty of Sciences, University of Yaounde 1, Cameroon

${ }^{\text {eg }}$ Bioversity International, Yaoundé, Cameroon

eh Faculty of Forestry, University of Toronto, Canada

ei Ministère des Eaux, Forêts, Chasse et Pêche (MEFCP), Bangui, Central African Republic

ej Universidad Católica de la Santísima Concepción, Chile

${ }^{\mathrm{ek}}$ Universidad de La Serena, Chile

${ }^{\text {el }}$ Research Institute of Tropical Forestry, Chinese Academy of Forestry, China

em Kunming Institute of Botany, Chinese Academy of Sciences, China

en Beijing Forestry University, China

eo Universidad Nacional Abierta y a Distancia, Red COL-TREE, Colombia

ep Corporación COL-TREE, Colombia

${ }^{\text {eq }}$ Nuevo Estándar Biotropical NEBIOT SAS, Colombia

er Universidad del Tolima, Colombia

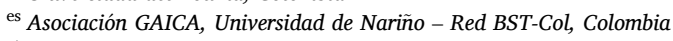

et Parques Nacionales Naturales, Territorial Caribe - Red BST-Col, Colombia

${ }^{\text {eu }}$ Universidad del Atlantico - Red BST-Col, Colombia

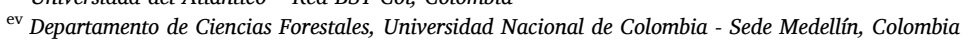

${ }^{\text {ew }}$ Socioecosistemas y Clima Sostenible, Fundacion con Vida, Colombia

ex Parques Nacionales Naturales de Colombia - Red BST-Col, Colombia

ey Instituto de Investigación de Recursos Biológicos Alexander von Humboldt-Red BST-Col, Colombia

${ }^{\text {ez }}$ UNAL, Colombia

fa Instituto de Investigación Recursos Biologicos Alexander von Humboldt - Red BST-Col, Colombia

fb Fundación Jardín Botánico de Medellín, Herbario "Joaquín Antonio Uribe” (JAUM) - Red BST-Col, Colombia

fc Universidad Nacional de Colombia sede Amazonia, Colombia

fd Coltree, Colombia

fe Facultad del Medio Ambiente y Recursos Naturales, Universidad Distrital Francisco José de Caldas - Red BST-Col, Colombia

${ }^{\mathrm{ff}}$ Universidad de Tolima, Colombia

${ }^{\mathrm{fg}}$ Fundación Orinoquia Biodiversa - Red BST-Col, Colombia 
fh Departamento de Biología, Facultad de Ciencias Naturales, Universidad Icesi - Red BST-Col, Colombia

fi Instituto de Ciencias Naturales, Universidad Nacional de Colombia, Colombia

${ }^{\mathrm{fj}}$ Universidad de los Llanos, Colombia

${ }^{\mathrm{fk}}$ Servicios Ecoysistemicos y Cambio Climatico (SECC) Fundación Con Vida \& Corporación COL-TREE, Colombia

${ }^{\mathrm{fl}}$ Universidad del Rosario, Colombia

${ }^{\mathrm{fm}}$ Fundacion Ecosistemas Secos de Colombia - Red BST-Col, Colombia

${ }^{\text {fn }}$ Universidad de los Andes - ANDES herbarium, Colombia

${ }^{\text {fo }}$ Institute of Botany, Czech Academy of Sciences, Czech Republic

${ }^{\mathrm{fp}}$ Palacky University, Czech Republic

${ }^{\mathrm{fq}}$ Czech University of Life Sciences, Prague, Czech Republic

${ }^{\mathrm{fr}}$ Mendel University, Brno, Czech Republic

${ }^{\mathrm{fs}}$ World Wide Fund for Nature, Democratic Republic of the Congo

${ }^{\mathrm{ft}}$ Wildlife Conservation Society-DR Congo, Democratic Republic of the Congo

${ }^{\mathrm{fu}}$ Lukuru Wildlife Research Foundation, Democratic Republic of the Congo

${ }^{\mathrm{fv}}$ Universite de Kisangani, Democratic Republic of the Congo

${ }^{\mathrm{fw}}$ Faculté des Sciences, Laboratoire d'écologie et aménagement forestier, Université de Kisangani, Kisangani, Democratic Republic of the Congo

${ }^{\mathrm{fx}}$ Université de Kisangani Faculté des Sciences Agronomiques République Démocratique du Congo, Democratic Republic of the Congo

${ }^{\mathrm{fy}}$ Ministère de l'Environnement et Développement Durable, Kinshasa, Democratic Republic of the Congo

${ }^{\mathrm{fz}}$ Aarhus University, Denmark

${ }^{\text {ga }}$ University of Copenhagen, Denmark

${ }^{\mathrm{gb}}$ Escuela Politécnica Nacional del Ecuador, Ecuador

${ }^{g c}$ Herbario Alfredo Paredes (QAP), Universidad Central del Ecuador, Ecuador

${ }^{g d}$ Universidad Técnica Particular de Loja, Ecuador

ge Grupo de Investigación en Biodiversidad, Medio Ambiente y Salud-BIOMAS, Universidad de las Américas, Campus Queri, Quito, Ecuador

${ }^{g f}$ Keller Science Action Center, The Field Museum, 1400 South Lake Shore Dr., Chicago, IL, USA

${ }^{g g}$ Universidad Estatal Amazónica, Facultad de Ingeniería Ambiental, Ecuador

${ }^{\text {gh }}$ Universidad Tecnica del Norte, Herbario Nacional del Ecuador, Ecuador

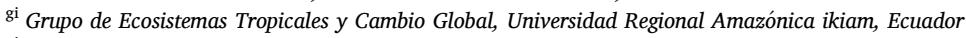

gj Colegio de Ciencias Biológicas y Ambientales COCIBA \& Extensión Galápagos, Universidad San Francisco de Quito-USFQ, Ecuador

${ }^{\mathrm{gk}}$ Herbario de Botánica Económica del Ecuador QUSF, Universidad San Francisco de Quito USFQ, Ecuador

${ }^{g 1}$ Galapagos Science Center, USFQ, UNC Chapel Hill, San Cristobal, Galapagos, Ecuador

${ }^{\mathrm{gm}}$ University of North Carolina-UNC Chapel Hill, USA

${ }^{\mathrm{gn}}$ University of Florida, Gainesville, USA

go FindingSpecies, Ecuador

gp Mekelle University, Ethiopia

${ }^{\mathrm{gq}}$ Independent Researcher, Ethiopia

${ }^{\mathrm{gr}}$ Environment, Climate Change and Coffee Forest Forum (ECCCFF), Ethiopia

${ }^{\mathrm{gs}}$ University of Turku, Finland

${ }^{g t}$ Centre de coopération International en Recherche Agronomique pour le Développement (CIRAD), France

gu $C N R S$, France

gv ONF, France

${ }^{\mathrm{gw}}$ INRAE, France

${ }^{\mathrm{gx}}$ Centre National de la Recherche Scientifique, France

${ }^{g y}$ AMAP, Univ Montpellier, IRD, CNRS, CIRAD, INRA, Montpellier, France

${ }^{\mathrm{g}}$ Forêts et Sociétés (F\&S), Centre de coopération International en Recherche Agronomique pour le Développement (CIRAD), Montpellier, France

${ }^{\text {ha }}$ Departement Hommes Natures Societes, Museum national d'histoire naturelle, France

${ }^{\mathrm{hb}}$ INRA, Kourou, French Guiana

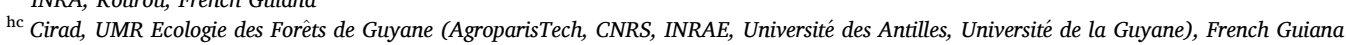

${ }^{\text {hd }}$ Ministry of Forests, Seas, Environment and Climate, Gabon

he Rougier-Gabon, Gabon

hf Agence Nationale des Parcs Nationaux Gabon, Gabon

hg Commission of Central African Forests (COMIFAC), Libreville, Gabon

${ }^{\text {hh }}$ Agence Nationale des Parcs Nationaux, Ministère des Forêts, des Eaux, de la Mer, de l'Environnement, Chargé du Plan Climat, des Objectifs de Développement Durable et du Plan d'Affectation des Terres, Gabon

hi Institut de Recherche en Ecologie Tropicale (CENAREST) Gabon/Agence Nationale des Parcs Nationaux, Gabon

hj Georg-August-University Göttingen, Germany

${ }^{\mathrm{hk}}$ University of Freiburg, Germany

${ }^{\mathrm{h} l}$ Institute of Botany, University of Hohenheim, 70593 Stuttgart, Germany

${ }^{\mathrm{hm}}$ Max Planck Institute for Chemistry, Germany

${ }^{\mathrm{hn}}$ Forestry Research Institute of Ghana (FORIG), Ghana

${ }^{\text {ho }}$ Mensuration Unit, Forestry Commission of Ghana, Ghana

hp Center for International Forestry Research, Guinea

ha Iwokrama International Centre for Rainforest Conservation and Development, Guyana

${ }^{\mathrm{hr}}$ Guyana Forestry Commission, Guyana

${ }^{\mathrm{h}}$ Utrecht University, Guyana

${ }^{\text {ht }}$ Centre for Sustainable Technologies, Indian Institute of Science, India

hu Centre for International Forestry Research (CIFOR), Indonesia

${ }^{\text {hv }}$ Herbarium Borgoriense, Indonesian Institute of Sciences (LIPI), Indonesia

${ }^{\mathrm{hw}}$ Indonesian Institute of Science, Bogor, Indonesia

${ }^{\mathrm{hx}}$ Forest Research and Development Agency (FORDA), Indonesia

hy Balitek-KSDA Samboja, Indonesia

${ }^{\mathrm{hz}}$ University of Florence and MUSE - Museo delle Scienze, Italy

${ }^{\text {ia }}$ Bioversity International, Italy

${ }^{\mathrm{ib}}$ Cirad, Cote d'Ivoire

${ }^{\text {ic }}$ Hokkaido University, Japan

${ }^{\text {id }}$ Graduate School of Agriculture, Kyoto University, Japan

ie Forestry Development Authority of the Government of Liberia (FDA), Liberia

if University of Liberia, Liberia

ig Sungai Wain Protection Forest, Malaysia

${ }^{\text {ih }}$ South East Asia Rainforest Research Partnership, Danum Valley Field Centre, Lahad Datu, Sabah, Malaysia 
ii Malaysian Palm Oil Board, Malaysia

ij Sabah Forestry Department, Forest Research Centre, Sandakan, Sabah, Malaysia

${ }^{\text {ik }}$ Universiti Malaysia Sabah, Malaysia

${ }^{i 1}$ Sabah Forestry Department, Malaysia

${ }^{i m}$ Sarawak Forestry Corporation, Malaysia

${ }^{\text {in }}$ Eduardo Mondlane University, Mozambique

${ }^{\text {io }}$ Herbarium UNAN-Leon, Universidad Nacional Autónoma de Nicaragua, Nicaragua

ip University of Abeokuta, Nigeria

${ }^{\text {iq }}$ Natural History Museum of Norway, Norway

${ }^{\text {ir }}$ University of Oslo, Norway

${ }^{\text {is }}$ Norwegian University of Life Sciences, Norway

it Universidad Nacional de la Amazonía Peruana (UNAP), Peru

iu Universidad Nacional de Jaén, Peru

iv Jardin Botanico de Missouri, Oxapampa, Peru

${ }^{\text {iw }}$ Andes to Amazon Biodiversity Program, Peru

${ }^{\text {ix }}$ Universidad Nacional de San Agustín de Arequipa, Peru

${ }^{\text {iy }}$ Facultad de Ciencias Biológicas, Universidad Nacional de la Amazonía Peruana, Peru

${ }^{\mathrm{iz}}$ Kené - Instituto de Estudios Forestales y Ambientales, Peru

ja Instituto de Investigaciones de la Amazonia Peruana (IIAP), Peru

${ }^{\mathrm{jb}}$ Universidad Nacional Jorge Basadre de Grohmann (UNJBG), Peru

${ }^{j c}$ Universidad Nacional de San Antonio Abad del Cusco, Peru

${ }^{\mathrm{j} d}$ Centro de Conservación, Investigación y Manejo, CIMA, Peru

${ }^{\text {je }}$ Pontificia Universidad Católica del Perú, Peru

${ }^{\text {jf }}$ Asociacion Bosques Perú, Peru

${ }^{\text {jg }}$ Université Officielle de Bukavu, Bukavu, Congo

${ }^{\text {jh }}$ Université Marien N'Gouabi, Brazzaville, Congo

${ }^{\mathrm{ji}}$ Wildlife Conservation Society, Congo

jj Ecole Nationale Supérieure d'Agronomie et de Foresterie, Université Marien Ngouabi, Congo

${ }^{\mathrm{jk}}$ Univeriste Marien Ngouabi, Congo

${ }^{\mathrm{j} 1}$ The Gola Rainforest National Park, Kenema, Sierra Leone

jm Department of Geography, National University of Singapore, Singapore

${ }^{j n}$ Departamento de Biología y Geología, Física y Química inorgánica, Universidad Rey Juan Carlos, Spain

jo Real Jardín Botánico - CSIC, Spain

${ }^{\mathrm{jp}}$ Departamento de Biología, Área de Botánica, Universidad Autónoma de Madrid, Spain

jq Museo Nacional de Ciencias Naturales (MNCN-CSIC), Spain

${ }^{\mathrm{jr}}$ Centre for Agricultural Research in Suriname (CELOS), Suriname

${ }^{\mathrm{j} s}$ Stockholm Environment Institute, Sweden

${ }^{\mathrm{jt}}$ Department of Plant Ecology and Evolution, Uppsala University, Sweden

ju Southern Swedish Forest Research Centre, Sweden

${ }^{\mathrm{jv}}$ InfoFlora, Conservatoire et Jardin Botanique Geneve, Switzerland

${ }^{\mathrm{jw}}$ National Chung Hsing University, Taiwan

${ }^{\mathrm{jx}}$ Sokoine University of Agriculture, Tanzania

jy Naturalis Biodiversity Center, The Netherlands

${ }^{\mathrm{jz}}$ Wageningen University, Forest Ecology and Forest Management Group, The Netherlands

ka Tropenbos International, The Netherlands

${ }^{\mathrm{kb}}$ Institute for Biodiversity and Ecosystem Dynamics, University of Amsterdam, The Netherlands

${ }^{\mathrm{kc}}$ Bureau Waardenburg BV, The Netherlands

${ }^{k d}$ Van Hall Larenstein University of Applied Sciences, The Netherlands

ke Van der Hout Forestry Consulting, The Netherlands

${ }^{\mathrm{kf}}$ Utrecht University, Domplein 29, 3512 JE Utrecht, The Netherlands

${ }^{\mathrm{kg}}$ PROMAB, The Netherlands

${ }^{\mathrm{kh}}$ Wageningen University, Plant Ecology and Nature Conservation Group, The Netherlands

${ }^{k i}$ Data for Sustainability, The Netherlands

${ }^{\mathrm{kj}}$ Utrecht University, The Netherlands

${ }^{\mathrm{kk}}$ Department of Zoology, Entomology \& Fisheries Sciences, Makerere University, Kampala, Uganda

${ }^{\mathrm{k} l}$ The Institute of Tropical Forest Conservation (ITFC), Mbarara University of Science and Technology (MUST), Mbarara, Uganda

$\mathrm{km}$ George Washington University, Uganda

${ }^{\mathrm{kn}}$ Makerere University, Kampala, Uganda

ko Department of Forestry, Biodiversity and Tourism, Makerere University, Kampala, Uganda

${ }^{\mathrm{kp}}$ Wildife Conservation Society, Uganda

${ }^{\mathrm{kq}}$ University of Stirling, United Kingdom

${ }^{\mathrm{kr}}$ University of Kent, United Kingdom

${ }^{\text {ks }}$ School of Geography, University of Leeds, U.K.

${ }^{\mathrm{kt}}$ UK Centre of Ecology \& Hydrology, United Kingdom

${ }^{\mathrm{ku}}$ Lancaster University, United Kingdom

${ }^{\mathrm{kv}}$ University of Oxford, United Kingdom

${ }^{\mathrm{kw}}$ The Landscapes and Livelihoods Group (TLLG), United Kingdom

${ }^{\mathrm{kx}}$ Overseas Development Institute, United Kingdom

${ }^{\text {ky }}$ Manchester Metropolitan University, United Kingdom

${ }^{\mathrm{kz}}$ University of Aberdeen, United Kingdom

${ }^{1 a}$ University of Exeter, United Kingdom

${ }^{1 \mathrm{~b}}$ School of GeoSciences, University of Edinburgh, United Kingdom

${ }^{1 c}$ University of Cambridge, United Kingdom

${ }^{\text {ld }}$ Department of Environment and Geography, University of York, United Kingdom

le Department of Geography, University College London, United Kingdom

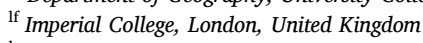

${ }^{1 g}$ School of Geography, Earth \& Environmental Sciences, Birmingham Institute of Forest Research, University of Birmingham, United Kingdom

${ }^{\text {lh }}$ University of Plymouth, United Kingdom

${ }^{\text {li }}$ Geography, College of Life and Environmental Sciences, University of Exeter, United Kingdom

${ }^{1 \mathrm{j}}$ Lancaster Environment Centre, Lancaster University, United Kingdom 
${ }^{1 \mathrm{k}}$ University of Edinburgh, United Kingdom

${ }^{11}$ School of Biology, University of Leeds, United Kingdom

${ }^{1 \mathrm{~m}}$ Royal Botanic Garden Edinburgh, United Kingdom

In CENAREST \& ANPN \& Stirling University, United Kingdom

${ }^{\text {lo }}$ University of Bristol, School of Biological Sciences, United Kingdom

${ }^{\mathrm{lp}}$ Department of Plant Sciences, University of Cambridge, United Kingdom

${ }^{\mathrm{la}}$ Living with Lake Centre, Laurentian University, Canada

${ }^{1 \mathrm{r}}$ Royal Botanic Gardens Kew, United Kingdom

${ }^{1 s}$ The Royal Society for the Protection of Birds, Centre for Conservation Science, Sandy, United Kingdom

${ }^{1 t}$ Environmental Change Institute, School of Geography and the Environment, University of Oxford, United Kingdom

${ }^{\text {lu }}$ UK Centre for Ecology \& Hydrology, United Kingdom

${ }^{1 v}$ School of Geography and the Environment, University of Oxford, United Kingdom

${ }^{\mathrm{lw}}$ The Royal Botanic Gardens, United Kingdom

${ }^{1 x}$ Department of Geography and Environmental Science, University of Dundee, United Kingdom

ly School of Biological Sciences, University of Southampton, United Kingdom

${ }^{1 \mathrm{z}}$ University of East Anglia, United Kingdom

ma Stirling University, United Kingdom

${ }^{\mathrm{mb}}$ School of Earth and Environment, University of Leeds, United Kingdom

${ }^{\mathrm{mc}}$ Department of Plant \& Soil Science, Cruickshank Building, School of Biological Sciences, University of Aberdeen, United Kingdom

${ }^{\mathrm{md}}$ Institute for Transport Studies, University of Leeds, United Kingdom

${ }^{m e}$ UK Research \& Innovation, United Kingdom

${ }^{\mathrm{mf}}$ University of Nottingham, United Kingdom

${ }^{\mathrm{mg}}$ University of Bangor, United Kingdom

${ }^{\mathrm{mh}}$ Center for Tropical Research, Institute of the Environment and Sustainability, University of California, Los Angeles, USA

mi Center for Tropical Conservation, Nicholas School of the Environment, Duke University, USA

${ }^{\mathrm{mj}}$ Ecology and Evolutionary Biology, University of Michigan, USA

${ }^{\mathrm{mk}}$ Saint Louis Zoo, USA

${ }^{\mathrm{ml}}$ Department of Organismic and Evolutionary Biology, Harvard University, USA

${ }^{\mathrm{mm}}$ Center for Global Discovery and Conservation Science, Arizona State University, USA

${ }^{\mathrm{m} n}$ Wildlife Conservation Society - Programme Congo, USA

${ }^{\mathrm{mo}}$ Woods Hole Research Center, USA

mp The University of Michigan Herbarium, USA

${ }^{\mathrm{mq}}$ Nicholas School of the Environment, USA

${ }^{\mathrm{mr}}$ National Park Service, USA

${ }^{\mathrm{ms}}$ University of California, USA

${ }^{\mathrm{mt}}$ ForestGEO, Smithsonian Tropical Research Institute, USA

${ }^{\mathrm{mu}}$ University of Texas at Austin, USA

${ }^{\mathrm{m}}$ Smithsonian Institute, USA

${ }^{\mathrm{mw}}$ Washington University in Saint Louis, Center for Conservation and Sustainable Development at the Missouri Botanical Garden, USA

${ }^{\mathrm{mx}}$ Smithsonian Tropical Research Institute, Smithsonian Institution Forest Global Earth Observatory (ForestGEO), USA

${ }^{\mathrm{my}}$ Forest Global Earth Observatory (ForestGEO), Smithsonian Tropical Research Institute, Washington, DC, USA

${ }^{\mathrm{mz}}$ George Mason University, VA, USA

${ }^{n a}$ Missouri Botanical Garden, USA

${ }^{\mathrm{nb}}$ Broward County Parks and Recreation, USA

${ }^{\mathrm{nc}}$ Nova Southeastern University, USA

${ }^{n d}$ Science and Education, The Field Museum, USA

ne Department of Biology, Boston University, USA

${ }^{\mathrm{nf}}$ Wake Forest University, USA

${ }^{\mathrm{ng}}$ Department of Geographical Sciences, University of Maryland, College Park, MD, USA

${ }^{\text {nh }}$ San Diego Zoo Institute for Conservation Research, USA

${ }^{\mathrm{ni}}$ Biology Department, Washington State University, Vancouver, WA, USA

${ }^{\mathrm{nj}}$ Yale School of Forestry \& Environmental Studies, USA

${ }^{\mathrm{nk}}$ Columbia University, USA

${ }^{\mathrm{nl}}$ Department of Environmental Science, Policy, and Management, University of California, Berkeley, USA

${ }^{\mathrm{nm}}$ School of Earth Sciences and Environmental Sustainability, Northern Arizona University, USA

${ }^{\mathrm{nn}}$ Department of Geography and the Environment, University of Texas at Austin, USA

${ }^{\text {no }}$ UNELLEZ-Guanare, Programa de Ciencias del Agro y el Mar, Herbario Universitario (PORT), Ci Progress GreenLife, Venezuela

${ }^{\mathrm{np}}$ Universidad Nacional Experimental de Guayana, Venezuela

${ }^{\mathrm{nq}}$ Instituto Venezolano de Investigaciones Científicas (IVIC), Venezuela

${ }^{\mathrm{nr}}$ Universidad de los Andes, Merida, Venezuela

${ }^{n s}$ Viet Nature Conservation Centre, Viet Nam

${ }^{n t}$ CIRAD, French Guiana

${ }^{\mathrm{n} u}$ INRAE, French Guiana

${ }^{n v}$ Center for International Forestry Research, Indonesia

${ }^{\mathrm{nw}}$ School of Life Sciences, University of Lincoln, U.K.

${ }^{\mathrm{nx}}$ A global collaborative hosted at the University of Leeds

${ }^{n y}$ Universidad Nacional Agraria La Molina (UNALM), Peru

\section{A R T I C L E I N F O}

Keywords:

Amazonia

Africa

Southeast Asia

Rainforest

RAINFOR

AfriTRON

Species richness

\section{A B S T R A C T}

Tropical forests are the most diverse and productive ecosystems on Earth. While better understanding of these forests is critical for our collective future, until quite recently efforts to measure and monitor them have been largely disconnected. Networking is essential to discover the answers to questions that transcend borders and the horizons of funding agencies. Here we show how a global community is responding to the challenges of tropical ecosystem research with diverse teams measuring forests tree-by-tree in thousands of long-term plots. We review the major scientific discoveries of this work and show how this process is changing tropical forest science. Our core approach involves linking long-term grassroots initiatives with standardized protocols and data 
Forest plots

Permanent sample plots

Monitoring

Dynamics

Carbon sink

Global change

Ecology

Biodiversity

management to generate robust scaled-up results. By connecting tropical researchers and elevating their status, our Social Research Network model recognises the key role of the data originator in scientific discovery. Conceived in 1999 with RAINFOR (South America), our permanent plot networks have been adapted to Africa (AfriTRON) and Southeast Asia (T-FORCES) and widely emulated worldwide. Now these multiple initiatives are integrated via ForestPlots.net cyber-infrastructure, linking colleagues from 54 countries across 24 plot networks. Collectively these are transforming understanding of tropical forests and their biospheric role. Together we have discovered how, where and why forest carbon and biodiversity are responding to climate change, and how they feedback on it. This long-term pan-tropical collaboration has revealed a large long-term carbon sink and its trends, as well as making clear which drivers are most important, which forest processes are affected, where they are changing, what the lags are, and the likely future responses of tropical forests as the climate continues to change. By leveraging a remarkably old technology, plot networks are sparking a very modern revolution in tropical forest science. In the future, humanity can benefit greatly by nurturing the grassroots communities now collectively capable of generating unique, long-term understanding of Earth's most precious forests.

Resumen: Los bosques tropicales son los ecosistemas más diversos y productivos del mundo y entender su funcionamiento es crítico para nuestro futuro colectivo. Sin embargo, hasta hace muy poco, los esfuerzos para medirlos y monitorearlos han estado muy desconectados. El trabajo en redes es esencial para descubrir las respuestas a preguntas que trascienden las fronteras y los plazos de las agencias de financiamiento. Aquí mostramos cómo una comunidad global está respondiendo a los desafíos de la investigación en ecosistemas tropicales a través de diversos equipos realizando mediciones árbol por árbol en miles de parcelas permanentes de largo plazo. Revisamos los descubrimientos más importantes de este trabajo y discutimos cómo este proceso está cambiando la ciencia relacionada a los bosques tropicales. El enfoque central de nuestro esfuerzo implica la conexión de iniciativas locales de largo plazo con protocolos estandarizados y manejo de datos para producir resultados que se puedan trasladar a múltiples escalas. Conectando investigadores tropicales, elevando su posición y estatus, nuestro modelo de Red Social de Investigación reconoce el rol fundamental que tienen, para el descubrimiento científico, quienes generan o producen los datos. Concebida en 1999 con RAINFOR (Suramérica), nuestras redes de parcelas permanentes han sido adaptadas en África (AfriTRON) y el sureste asiático (TFORCES) y ampliamente replicadas en el mundo. Actualmente todas estas iniciativas están integradas a través de la ciber-infraestructura de ForestPlots.net, conectando colegas de 54 países en 24 redes diferentes de parcelas. Colectivamente, estas redes están transformando nuestro conocimiento sobre los bosques tropicales y el rol de éstos en la biósfera. Juntos hemos descubierto cómo, dónde y porqué el carbono y la biodiversidad de los bosques tropicales está respondiendo al cambio climático y cómo se retroalimentan. Esta colaboración pan-tropical de largo plazo ha expuesto un gran sumidero de carbono y sus tendencias, mostrando claramente cuáles son los factores más importantes, qué procesos se ven afectados, dónde ocurren los cambios, los tiempos de reacción y las probables respuestas futuras mientras el clima continúa cambiando. Apalancando lo que realmente es una tecnología antigua, las redes de parcelas están generando una verdadera y moderna revolución en la ciencia tropical. En el futuro, la humanidad puede beneficiarse enormemente si se nutren y cultivan comunidades de investigadores de base, actualmente con la capacidad de generar información única y de largo plazo para entender los que probablemente son los bosques más preciados de la tierra.

Resumo: Florestas tropicais são os ecossistemas mais diversos e produtivos da Terra. Embora uma boa compreensão destas florestas seja crucial para o nosso futuro coletivo, até muito recentemente os esforços de medições e monitoramento tem sido amplamente desconexos. É essencial formarmos redes para obtermos respostas que transcendam as fronteiras e horizontes das agências financiadoras. Neste estudo nós mostramos como uma comunidade global está respondendo aos desafios da pesquisa de ecossistemas tropicais, com equipes diversas medindo florestas, árvore por árvore, em milhares de parcelas monitoradas a longo prazo. Nós revisamos as maiores descobertas científicas deste esforço global, e mostramos também como este processo vem mudando a ciência de florestas tropicais. Nossa abordagem principal envolve unir iniciativas de base a protocolos padronizados e gerenciamento de dados a fim de gerar resultados robustos em grandes escalas. Ao conectar pesquisadores tropicais e elevar seus status, nosso modelo de Rede de Pesquisa Social reconhece o papel chave do produtor dos dados na descoberta científica. Concebida em 1999 com o RAINFOR (América do Sul), nossa rede de parcelas permanentes foi adaptada para África (AfriTRON) e Sudeste Asiático (T-FORCES), e tem sido extensamente reproduzida em todo o mundo. Agora estas múltiplas iniciativas estão integradas através da infraestrutura cibernética do ForestPlots.net, conectando colegas de 54 países e 24 redes de parcelas. Estas iniciativas estão transformando coletivamente o entendimento das florestas tropicais e seus papéis na biosfera. Juntos nós descobrimos como, onde e por que o carbono e a biodiversidade da floresta estão respondendo às mudanças climáticas, e seus efeitos de retroalimentação. Esta duradoura colaboração pantropical revelou um grande sumidouro de carbono persistente e suas tendências, assim como tem evidenciado quais os fatores que influenciam essas tendências, quais processos florestais são mais afetados, onde eles estão mudando, seus atrasos no tempo de resposta, e as prováveis respostas das florestas tropicais conforme o clima continua a mudar. Dessa forma, aproveitando uma notável tecnologia antiga, redes de parcelas acendem as faíscas de uma moderna revolução na ciência das florestas tropicais. No futuro a humanidade pode se beneficiar incentivando estas comunidades locais que agora são coletivamente capazes de gerar conhecimentos únicos e duradouros sobre as florestas mais preciosas da Terra.

Résume: Les forêts tropicales sont les écosystèmes les plus diversifiés et les plus productifs de la planète. Si une meilleure compréhension de ces forêts est essentielle pour notre avenir collectif, jusqu'à tout récemment, les efforts déployés pour les mesurer et les surveiller ont été largement déconnectés. La mise en réseau est essentielle pour découvrir les réponses à des questions qui dépassent les frontières et les horizons des organismes de financement. Nous montrons ici comment une communauté mondiale relève les défis de la recherche sur les écosystèmes tropicaux avec diverses équipes qui mesurent les forêts arbre après arbre dans de milliers de parcelles permanentes. Nous passons en revue les principales découvertes scientifiques de ces travaux et montrons comment ce processus modifie la science des forêts tropicales. Notre approche principale consiste à relier les initiatives de base à long terme à des protocoles standardisés et une gestion de données afin de générer des résultats solides à grande échelle. En reliant les chercheurs tropicaux et en élevant leur statut, notre modèle de 
réseau de recherche sociale reconnaît le rôle clé de l'auteur des données dans la découverte scientifique. Conçus en 1999 avec RAINFOR (Amérique du Sud), nos réseaux de parcelles permanentes ont été adaptés à l'Afrique (AfriTRON) et à l'Asie du Sud-Est (T-FORCES) et largement imités dans le monde entier. Ces multiples initiatives sont désormais intégrées via l'infrastructure ForestPlots.net, qui relie des collègues de 54 pays à travers 24 réseaux de parcelles. Ensemble, elles transforment la compréhension des forêts tropicales et de leur rôle biosphérique. Ensemble, nous avons découvert comment, où et pourquoi le carbone forestier et la biodiversité réagissent au changement climatique, et comment ils y réagissent. Cette collaboration pan-tropicale à long terme a révélé un important puits de carbone à long terme et ses tendances, tout en mettant en évidence les facteurs les plus importants, les processus forestiers qui sont affectés, les endroits où ils changent, les décalages et les réactions futures probables des forêts tropicales à mesure que le climat continue de changer. En tirant parti d'une technologie remarquablement ancienne, les réseaux de parcelles déclenchent une révolution très moderne dans la science des forêts tropicales. À l'avenir, l'humanité pourra grandement bénéficier du soutien des communautés de base qui sont maintenant collectivement capables de générer une compréhension unique et à long terme des forêts les plus précieuses de la Terre.

Abstrak: Hutan tropika adalah di antara ekosistem yang paling produktif dan mempunyai kepelbagaian biodiversiti yang tinggi di seluruh dunia. Walaupun pemahaman mengenai hutan tropika amat penting untuk masa depan kita, usaha-usaha untuk mengkaji dan mengawas hutah-hutan tersebut baru sekarang menjadi lebih diperhubungkan. Perangkaian adalah sangat penting untuk mencari jawapan kepada soalan-soalan yang menjangkaui sempadan dan batasan agensi pendanaan. Di sini kami menunjukkan bagaimana sebuah komuniti global bertindak balas terhadap cabaran penyelidikan ekosistem tropika melalui penglibatan pelbagai kumpulan yang mengukur hutan secara pokok demi pokok dalam beribu-ribu plot jangka panjang. Kami meninjau semula penemuan saintifik utama daripada kerja ini dan menunjukkan bagaimana proses ini sedang mengubah bidang sains hutan tropika. Teras pendekatan kami memberi tumpuan terhadap penghubungan inisiatif akar umbi jangka panjang dengan protokol standar serta pengurusan data untuk mendapatkan hasil skala besar yang kukuh. Dengan menghubungkan penyelidik-penyelidik tropika dan meningkatkan status mereka, model Rangkaian Penyelidikan Sosial kami mengiktiraf kepentingan peranan pengasas data dalam penemuan saintifik. Bermula dengan pengasasan RAINFOR (Amerika Selatan) pada tahun 1999, rangkaian-rangkaian plot kekal kami kemudian disesuaikan untuk Afrika (AfriTRON) dan Asia Tenggara (T-FORCES) dan selanjutnya telah banyak dicontohi di seluruh dunia. Kini, inisiatif-inisiatif tersebut disepadukan melalui infrastruktur siber ForestPlots. net yang menghubungkan rakan sekerja dari 54 negara di 24 buah rangkaian plot. Secara kolektif, rangkaian ini sedang mengubah pemahaman tentang hutan tropika dan peranannya dalam biosfera. Kami telah bekerjasama untuk menemukan bagaimana, di mana dan mengapa karbon serta biodiversiti hutan bertindak balas terhadap perubahan iklim dan juga bagaimana mereka saling bermaklum balas. Kolaborasi pan-tropika jangka panjang ini telah mendedahkan sebuah sinki karbon jangka panjang serta arah alirannya dan juga menjelaskan pemandupemandu perubahan yang terpenting, di mana dan bagaimana proses hutan terjejas, masa susul yang ada dan kemungkinan tindakbalas hutan tropika pada perubahan iklim secara berterusan di masa depan. Dengan memanfaatkan pendekatan lama, rangkaian plot sedang menyalakan revolusi yang amat moden dalam sains hutan tropika. Pada masa akan datang, manusia sejagat akan banyak mendapat manfaat jika memupuk komuniti-komuniti akar umbi yang kini berkemampuan secara kolektif menghasilkan pemahaman unik dan jangka panjang mengenai hutan-hutan yang paling berharga di dunia.

\section{Introduction}

As the most diverse and productive ecosystems on Earth, tropical forests play essential roles in the carbon and water cycles and maintenance of global biodiversity. Tropical forest lands are also home to more than a billion people and thousands of cultures. Having first provided the environments and germplasm that sustained foragers and farmers since the earliest days of humanity, today they underpin a large fraction of our globalized diet and intense demand for water, food and clean air. They also affect our health in multiple ways, providing rich pharmacopoeias to traditional and modern societies, and capable of changing the course of history when pandemic zoonotic pathogens emerge as forests and wildlife are exploited. Tropical forests are also critical to determining the degree and impact of anthropogenic climate change. Because of their extent, carbon density and productivity, they may both slow global heating by absorbing carbon into their biomass and soils, or accelerate it as deforestation and high temperatures damage forests and release carbon to the atmosphere.

Tropical carbon and biodiversity are therefore critical targets for environmental measurement and monitoring. While vital to our past and future, efforts to measure and monitor them have until recently been localised and largely disconnected. Although aspects of their ecology can be sensed remotely, on-the-ground, tree-by-tree measurement is essential. Indeed ground measurements are irreplaceable - whether to address a plethora of ecological questions (e.g., Wright, 2021), inform and validate ecosystem models (e.g., Malhi et al., 2021), or assist with interpreting remotely acquired data (e.g., Chave et al., 2019; Duncanson et al., 2019; Phillips et al., 2019). Yet the very features that enhance tropical forests' ecological value, such as remoteness, diversity and high rainfall, make fieldwork challenging. Tropical forest science and scientists from forest-rich countries are often under-resourced and academically marginalised. Often colonized from afar and distant from economic centres, tropical nature and many who explore it remain peripheral to national and global academic and political priorities.

The focus of this paper is specifically about the power of new collaborative networks to transform tropical forest science - what we do, how we do it, and eventually who does it - to understand tropical forest functioning and dynamics over large temporal and spatial scales. Conceived and funded starting in South America in 1999 (RAINFOR, Malhi et al., 2002) and later adapted to Africa (AfriTRON, Lewis et al., 2009) and Southeast Asia (T-FORCES, Qie et al., 2017) our approach encourages international grassroots initiatives and links them with standardized field methods and data management. Now, with ForestPlots.net (López-Gonzalez et al., 2011, 2015) we support multiple networks with cyber-infrastructure that enables tropical scientists to do together what was previously impossible alone. Providing tools to ensure tropical scientists can manage, share and analyse their data themselves, ForestPlots.net is a global platform where data originators are in control and free to collaborate, support, or lead as much as they like. However, while much has been accomplished the wider challenges still run deep. Our aim of supporting the best possible science within a model of equitable access to data and other resources remains as much 
an aspiration as a claim of achievements already made.

Here we first review how the continental networks and ForestPlots.net emerged, in terms of collaborators, institutions, people and plots. Next we focus on key scientific achievements of the combined networks, including a comprehensive understanding of the variation in biomass carbon stock, growth rates, and carbon residence time among continents. Wealso review multiple discoveries concerning large-scale changes over time, with insights emerging from highly distributed permanent plots that have transformed our understanding of the role that tropical forests play in the biosphere. Finally, we return to the challenges of building and sustaining long-term science networks in the tropics and outline key priorities for the future.

\section{Network development}

Tropical research plots that tag, measure, identify and follow forests tree-by-tree have existed for decades. They long precede any continental or global network, but no plot survives since before 1939 and few predate 1970. The earliest efforts were closely connected to the imperial and post-imperial projects of European nations. As such, these were largely motivated by questions of timber inventory and wood production, and only later diversity and wider ecological questions. The very first permanent sample plots we are aware of in the tropics were installed in 1857 by the German forester Brandis, who worked for the British in Burma (now Myanmar) and later in other parts of India (Dawkins and Philip, 1998). In India a few extant Forest Department plots date to 1939 (Pomeroy et al., 2003). Important early work in Southeast Asia included plots installed by Don Nicholson and J.E.D. Fox in the 1950s through to the 1970s, as well as Peter Ashton since the 1960s and John Proctor since the 1970s. In Africa, early permanent plots include those installed by William Eggeling in Uganda in the 1930s. Among plots surviving today are one in Mpanga Forest, Uganda, set up by Alan Hamilton in 1968, and those established by Mike Swaine in Ghana and Hans Woell in Liberia in the 1970s. Later plots were established by Jan Reistma and Lee White (Gabon), Bonaventure Sonké (Cameroon), Kofi Affum Baffoe (Ghana), and Henri-Félix Maître and colleagues (Gabon, Congo, C.A.R.). In Australia, North Queensland saw the first plot sampling, for timber, in the 1930s, with many sites from the 1970s still maintained today by the national science agency (CSIRO). Separately Joe Connell, co-originator of the influential Janzen-Connell hypothesis, installed and expanded long-term ecological plots in 1963.

In the tropical Americas, T.A.W. Davis and Paul Richards installed ecological plots in Guyana in the 1930s (Davis and Richards, 1933) but these do not survive, while Frank Wadsworth established long-term plots in Puerto Rico's subtropical forests starting in 1943 (e.g. Drew et al., 2009). In Suriname, Schulz and colleagues established silvicultural studies in the 1950s and 60s that were used to design the CELOS Management System (Werger, 2011). Neotropical ecological plots that persist today include many in Venezuela by Jean-Pierre Veillon in the 1950s, 60s and 70s (Vilanova et al., 2018) and Rafael Herrera, Ernesto Medina and colleagues in the 1970s, as well as a few in Brazilian Amazonia by João Murça Pires, H. Dobzhansky and G.A. Black and later Ghillean Prance, and several in Costa Rica since 1969 by Diana and Milton Lieberman. Elsewhere, Alwyn Gentry, John Terborgh, Terry Erwin, Gary Hartshorn, David Neill and Rodolfo Vásquez set up the first long-term plots in western Amazon in the late 1970s and 80s (Gentry, 1988a; Monteagudo Mendoza et al., 2020). Eastern and central Amazon plots survive established by Samuel Almeida, Ima Vieira and Rafael Salomão in Pará (Salomão, 1991; Pires and Salomão, 2000), Tom Lovejoy, Niro Higuchi and colleagues near Manaus, Henri-Félix Maître in French Guiana, and Marcelo Nascimento and colleagues in Roraima. The earliest extant plots in southern Amazonia originated with Tim Killeen, Luzmila Arroyo, Beatriz Marimon and José Roberto Rodrigues. The first long-term tropical large plot was established in Costa Rica (Hubbell, 1979), which represented a separate innovation that permitted plot-level analysis of multi-species demography, followed soon after by the first 50-ha plot in Panama (Hubbell and Foster, 1983; Wright, 2021) and later developments by the Smithsonian Institution and the ForestGEO network (e.g. Anderson-Teixeira et al., 2015).

RAINFOR (Red Amazónica de Inventarios Forestales) is the first international tropical forest network encompassing highly distributed long-term plots. RAINFOR was inspired by Alwyn Gentry, a virtuoso tropical botanist who established the first globally standardized floristic inventories. In the 1970s Gentry developed a 0.1-ha sampling design to rapidly inventory diversity in species-rich tropical forests, capturing all stems $\geq 2.5 \mathrm{~cm}$ diameter. He and his colleagues applied it throughout the tropical Americas as well as parts of Africa, India, Southeast Asia, Australasia, and some northern and southern temperate forests. By the time of his untimely death at the age of 48 in 1993, Gentry had completed 226 of these samples, comprising an inventory of thousands of tree and liana species including many new to science. His legacy lives on in multiple ways. After studying with Walter Lewis and recruited by Peter Raven in the early 1970s, Gentry was a key figure in the Missouri Botanical Garden's golden age of tropical botany. He collected $>80,000$ plant specimens, approximately half of which are tropical trees and lianas. He pioneered a new approach to the challenge of identifying plants in the world's most diverse forests (Gentry and Vasquez, 1993) that has inspired generations of botanists throughout Latin America. Perhaps most importantly, it was Gentry who embodied the ambition of combining efficient ecological sampling with high-quality identifications and replicating these to create highly distributed measurements of the world's forests (e.g. Gentry, 1988b; Clinebell et al., 1995; Phillips and Miller, 2002; Phillips and Raven, 1997). He also established permanent plots (Gentry, 1988a) that feature in the first continental and pan-tropical analyses of forest carbon and dynamics (Phillips and Gentry, 1994; Phillips et al., 1994; Phillips et al., 1998), which in turn led to the creation of RAINFOR (Malhi et al., 2002; López-González and Phillips, 2012) and its protocols (e.g. Phillips et al., 2002). Originating in 1999 from a small nucleus of researchers and plots and supported by EU funding to Brazil's LBA initiative and UK scientists, RAINFOR grew to tackle the challenge of analysing Amazonian forests and climate responses tree-by-tree from the ground up. By bringing different groups together RAINFOR facilitated the development of long-term international collaborations to measure and understand not only forest dynamics and diversity but also biogeochemistry and carbon fluxes.

While RAINFOR has grown steadily, other plot networks later emerged with complementary foci in South America. Some are daughter initiatives to RAINFOR, others were formed separately, but most share a similar ethos and strongly overlapping protocols. To the extent that they can be combined together these networks represent an impressive Observatory for Neotropical Forests. Below we report key information about many vibrant networks worldwide that specifically contribute to ForestPlots.net (Table 1), while here we briefly enumerate national and international neotropical networks, the majority of which ForestPlots.net supports. These include (with dates when plots were censused or consolidated as a network) Tropical Ecology Assessment and Monitoring (TEAM, 2002), Amazon Tree Diversity Network (ATDN, 2003; ter Steege et al., 2003), Programa de Pesquisa em Biodiversidade (PPBio, 2004, Brazil), Red Colombiana de Monitoreo de los Bosques (COL-TREE, 2004), Global Ecosystems Monitoring (GEM, 2010; Malhi et al., 2021), Latin American Seasonally Dry Tropical Forest Network (DryFlor, 2012), Red de Investigación y Monitoreo del Bosque Seco Tropical en Colombia (Red BST-Col, 2014), Secondary Forest Network (2ndFOR, 2015), Peru Monitoring Network (MonANPerú, 2017), sANDES (Tree Diversity, Composition and Carbon in Andean Montane Forests, 2019), and Red de Bosques Andinos (RBA, 2020), as well as global networks and meta-networks including ForestGEO (Anderson-Teixeira et al., 2015), GFBI (Steidinger et al., 2019), sPlot (Bruelheide et al., 2019), FOS (Schepaschenko et al., 2019) and TmFO in logged forests (Sist et al., 2015). Each of these has notable achievements of their own and at the time of writing this article in 2020 almost all have active research programmes. 
Table 1

Networks contributing to ForestPlots.net.

We report the 24 international, national, and regional plot networks contributing to and supported by ForestPlots.net in 2020, in order of date of affiliation. Note that some plots contribute to more than one network, in some cases the plots managed at ForestPlots.net are fewer than the total number of plots of the network, while others are not 'networked' but managed by individual researchers. Hence, cross-network totals do not correspond precisely to the number of plots managed. We include 20 tropical networks with multi-census plots plus four large-scale floristic-focussed scientific networks (ATDN, CAO, sANDES, RedGentry) that work exclusively with single-census data. All numbers compiled September 2020. As an open collaborative project ForestPlots.net welcomes all contributors with carefullymanaged plots.

\begin{tabular}{|c|c|c|c|c|c|c|c|c|c|c|}
\hline Network $^{\mathrm{a}}$ & Geography & $\begin{array}{l}\text { Main } \\
\text { purposes }^{b}\end{array}$ & $\begin{array}{l}\text { Joined } \\
\text { ForestPlot } \\
\text { s.net }\end{array}$ & $\begin{array}{l}\text { Initiated [e.g. } \\
\text { plots censused } \\
\text { as a network] }\end{array}$ & $\begin{array}{l}\text { First census } \\
\text { in Forest } \\
\text { Plots.net }\end{array}$ & $\begin{array}{l}\mathrm{n} \text { (plots in } \\
\text { ForestPlot } \\
\text { s.net) }\end{array}$ & $\begin{array}{l}\mathrm{n} \text { (plots } \\
\text { recensued) }\end{array}$ & Modal plot & $\begin{array}{l}\text { Mean } \\
\text { size } \\
\text { (ha) }\end{array}$ & $\begin{array}{l}\text { Mean } \\
\text { (maximum) } \\
\text { years monitored }\end{array}$ \\
\hline RAINFOR & $\begin{array}{l}\text { South America: } \\
\text { tropical forests }\end{array}$ & $\begin{array}{l}\mathrm{B}, \mathrm{D}, \mathrm{F}, \mathrm{M}, \mathrm{T}, \\
\mathrm{V}\end{array}$ & 2000 & 2000 & 1961 & 593 & 427 & $\begin{array}{l}\text { 1-ha, } \\
>10 \mathrm{~cm} \mathrm{~d}\end{array}$ & 0.8 & $15(56)$ \\
\hline DBTV & $\begin{array}{l}\text { Venezuela: } \\
\text { tropical forests }\end{array}$ & $\mathrm{B}, \mathrm{D}, \mathrm{M}, \mathrm{T}$ & 2004 & 1956 & 1961 & 48 & 48 & $\begin{array}{l}\text { 0.25-ha, } \\
>10 \mathrm{~cm} \mathrm{~d}\end{array}$ & 0.25 & $30(55)$ \\
\hline COL-TREE & Colombia & $\begin{array}{l}\mathrm{B}, \mathrm{D}, \mathrm{F}, \mathrm{H}, \mathrm{M}, \\
\mathrm{R}, \mathrm{V}\end{array}$ & 2004 & 2004 & 1992 & 61 & 55 & $\begin{array}{l}\text { 1-ha, } \\
>10 \mathrm{~cm} \mathrm{~d}\end{array}$ & 0.8 & $9(25)$ \\
\hline TROBIT & $\begin{array}{l}\text { Pantropical: } \\
\text { forest-savanna } \\
\text { transition }\end{array}$ & $\mathrm{B}, \mathrm{D}, \mathrm{F}, \mathrm{H}, \mathrm{R}, \mathrm{T}$ & 2006 & 2006 & 2006 & 58 & 49 & $\begin{array}{l}\text { 1-ha, } \\
>10 \mathrm{~cm} \mathrm{~d}\end{array}$ & 1 & 12 \\
\hline AfriTRON & $\begin{array}{l}\text { Africa: tropical } \\
\text { forests }\end{array}$ & $\mathrm{B}, \mathrm{D}, \mathrm{F}, \mathrm{M}$ & 2009 & 2009 & 1939 & 575 & 407 & $\begin{array}{l}\text { 1-ha, } \\
>10 \mathrm{~cm} \mathrm{~d}\end{array}$ & 0.9 & 11 (69) \\
\hline ABERG & $\begin{array}{l}\text { Peru Andes: } \\
\text { Kosñipata Valley }\end{array}$ & $\mathrm{B}, \mathrm{D}, \mathrm{F}, \mathrm{M}, \mathrm{P}, \mathrm{T}$ & 2011 & 2011 & 2003 & 23 & 23 & $\begin{array}{l}\text { 1-ha, } \\
>10 \mathrm{~cm} \mathrm{~d}\end{array}$ & 1 & $12(16)$ \\
\hline T-FORCES & $\begin{array}{l}\text { Southeast Asia: } \\
\text { tropical forests }\end{array}$ & $\mathrm{B}, \mathrm{D}, \mathrm{F}, \mathrm{H}, \mathrm{M}$ & 2012 & 2012 & 1958 & 95 & 71 & $\begin{array}{l}\text { 1-ha, } \\
>10 \mathrm{~cm} \mathrm{~d}\end{array}$ & 1.3 & $22(56)$ \\
\hline GEM & Worldwide & $\begin{array}{l}\mathrm{D}, \mathrm{H}, \mathrm{M}, \mathrm{P}, \mathrm{R}, \\
\mathrm{T}\end{array}$ & 2012 & 2010 & 2010 & 53 & 45 & $\begin{array}{l}\text { 1-ha, } \\
>10 \mathrm{~cm} \mathrm{~d}\end{array}$ & 0.8 & $5(16)$ \\
\hline PELD-TRAN & $\begin{array}{l}\text { Brazil: Amazon- } \\
\text { Cerrado transition }\end{array}$ & $\begin{array}{l}\mathrm{B}, \mathrm{D}, \mathrm{F}, \mathrm{H}, \mathrm{M}, \\
\mathrm{R}, \mathrm{T}, \mathrm{V}\end{array}$ & 2012 & 2010 & 1996 & 48 & 45 & $\begin{array}{l}\text { 1-ha, } \\
>10 \mathrm{~cm} \mathrm{~d}\end{array}$ & 1 & $9(22)$ \\
\hline DRYFLOR & $\begin{array}{l}\text { Latin America and } \\
\text { Caribbean dry } \\
\text { forests }\end{array}$ & $\begin{array}{l}\mathrm{B}, \mathrm{D}, \mathrm{F}, \mathrm{H}, \mathrm{M}, \\
\mathrm{R}, \mathrm{T}, \mathrm{V}\end{array}$ & 2013 & 2012 & 2007 & 39 & 8 & $\begin{array}{l}\text { 0.5-ha, } \\
>5 \mathrm{~cm} \mathrm{~d}\end{array}$ & 0.3 & $7(8)$ \\
\hline ATDN & $\begin{array}{l}\text { Amazonia: } \\
\text { tropical forests }\end{array}$ & $\mathrm{F}, \mathrm{V}$ & 2014 & 2003 & 1974 & 413 & N/A & $\begin{array}{l}\text { 1-ha, } \\
>10 \mathrm{~cm} \mathrm{~d}\end{array}$ & 1 & N/A \\
\hline PPBio & $\begin{array}{l}\text { Brazil: forests and } \\
\text { savanna }\end{array}$ & $\begin{array}{l}\mathrm{B}, \mathrm{D}, \mathrm{F}, \mathrm{H}, \mathrm{M}, \\
\mathrm{T}, \mathrm{V}\end{array}$ & 2015 & 2004 & 2000 & 277 & 205 & $\begin{array}{l}\text { 1-ha, } \\
>10 \mathrm{~cm} \mathrm{~d}^{\mathrm{c}}\end{array}$ & 0.9 & $7(17)$ \\
\hline BIOTA & $\begin{array}{l}\text { Brazil: São Paulo } \\
\text { state, Atlantic } \\
\text { forests }\end{array}$ & $\begin{array}{l}\mathrm{B}, \mathrm{D}, \mathrm{F}, \mathrm{H}, \mathrm{M}, \\
\mathrm{P}, \mathrm{R}, \mathrm{T}, \mathrm{V}\end{array}$ & 2016 & 2005 & 2005 & 20 & 18 & $\begin{array}{l}\text { 1-ha, } \\
>10 \mathrm{~cm} \mathrm{~d}\end{array}$ & 0.9 & 11 (14) \\
\hline FATE & $\begin{array}{l}\text { Brazil: Amazon } \\
\text { fire-impacted }\end{array}$ & $\begin{array}{l}\text { B,D,H,M,R, } \\
\text { S,T }\end{array}$ & 2016 & 2014 & 2009 & 57 & 38 & $\begin{array}{l}0.25-\mathrm{ha} \\
>10 \mathrm{~cm} \mathrm{~d}^{\mathrm{c}}\end{array}$ & 0.3 & $4(10)$ \\
\hline RAS & Brazil: Para state & $\begin{array}{l}\text { B,D,F,H,M, } \\
\text { P,R,T,U,V }\end{array}$ & 2016 & 2009 & 1999 & 256 & 59 & $\begin{array}{l}0.25-\mathrm{ha} \\
>10 \mathrm{~cm} \mathrm{~d}^{\mathrm{c}}\end{array}$ & 0.26 & $6(20)$ \\
\hline MonANPeru & Peru & $\begin{array}{l}\mathrm{B}, \mathrm{D}, \mathrm{F}, \mathrm{H}, \mathrm{M}, \\
\mathrm{R}, \mathrm{U}, \mathrm{V}\end{array}$ & 2017 & 2017 & 1974 & 128 & 103 & $\begin{array}{l}\text { 1-ha, } \\
>10 \mathrm{~cm} \mathrm{~d}\end{array}$ & 1 & $15(43)$ \\
\hline Nordeste & $\begin{array}{l}\text { Brazil: Caatinga } \\
\text { biome }\end{array}$ & $\begin{array}{l}\mathrm{B}, \mathrm{D}, \mathrm{F}, \mathrm{H}, \mathrm{M}, \\
\mathrm{R}, \mathrm{T}\end{array}$ & 2017 & 2017 & 2017 & 33 & 3 & $\begin{array}{l}\text { 0.5-ha, } \\
>10 \mathrm{~cm} \mathrm{~d}\end{array}$ & 0.5 & 3 \\
\hline SEOSAW & $\begin{array}{l}\text { Southern Africa } \\
\text { woodlands }\end{array}$ & $\begin{array}{l}\text { B,D,F,H,M, } \\
\text { R,S,T,U,V }\end{array}$ & 2018 & 2018 & 2006 & 113 & 98 & $\begin{array}{l}\text { 1-ha, } \\
>5 \mathrm{~cm} \mathrm{~d}\end{array}$ & 0.5 & $9(15)$ \\
\hline Red BST-Col & $\begin{array}{l}\text { Colombia: dry } \\
\text { forests }\end{array}$ & $\begin{array}{l}\text { B,D,F,H,M, } \\
\text { R,U,V }\end{array}$ & 2018 & 2014 & 2014 & 11 & 1 & $\begin{array}{l}\text { 1-ha, } \\
>2.5 \mathrm{~cm} \mathrm{~d}\end{array}$ & 1 & $3(3)$ \\
\hline CAO & $\begin{array}{l}\text { Peru Amazon- } \\
\text { Andes }\end{array}$ & $\mathrm{B}, \mathrm{F}, \mathrm{S}, \mathrm{T}, \mathrm{V}$ & 2019 & 2009 & 2009 & 276 & N/A & $\begin{array}{l}0.28-\mathrm{ha} \\
>5 \mathrm{~cm} \mathrm{~d}\end{array}$ & 0.28 & N/A \\
\hline RedSPP & $\begin{array}{l}\text { Argentina: } \\
\text { subtropical }\end{array}$ & $\begin{array}{l}\mathrm{B}, \mathrm{D}, \mathrm{F}, \mathrm{H}, \mathrm{M}, \\
\mathrm{R}, \mathrm{V}\end{array}$ & 2019 & 2019 & 1992 & 16 & 7 & $\begin{array}{l}\text { 1-ha, } \\
>10 \mathrm{~cm} \mathrm{~d}\end{array}$ & 1.4 & $10(25)$ \\
\hline RBA & $\begin{array}{l}\text { South America: } \\
\text { Andean forests }\end{array}$ & $\begin{array}{l}\mathrm{B}, \mathrm{D}, \mathrm{F}, \mathrm{H}, \mathrm{M}, \\
\mathrm{R}, \mathrm{V}\end{array}$ & 2020 & 2012 & 1992 & 46 & 34 & $\begin{array}{l}\text { 1-ha, } \\
>10 \mathrm{~cm} \mathrm{~d}\end{array}$ & 1 & $11(25)$ \\
\hline SANDES & $\begin{array}{l}\text { South America: } \\
\text { Andean forests }\end{array}$ & $\mathrm{B}, \mathrm{F}, \mathrm{V}$ & 2020 & 2019 & 2003 & 191 & N/A & $\begin{array}{l}\text { 0.1-ha, } \\
>2.5 \mathrm{~cm} \mathrm{~d}\end{array}$ & 0.4 & N/A \\
\hline AfriMont & $\begin{array}{l}\text { Africa: tropical } \\
\text { montane forests }\end{array}$ & $\mathrm{B}, \mathrm{H}, \mathrm{M}, \mathrm{U}, \mathrm{V}$ & 2020 & 2020 & 1939 & 105 & N/A & $\begin{array}{l}\text { 1-ha, } \\
>10 \mathrm{~cm} \mathrm{~d}\end{array}$ & 0.6 & $10(69)$ \\
\hline RedGentry & $\begin{array}{l}\text { South America: } \\
\text { Amazon forests }\end{array}$ & $\mathrm{F}, \mathrm{V}$ & 2020 & 2020 & 1983 & 350 & N/A & $\begin{array}{l}\text { 0.1-ha, } \\
>2.5 \mathrm{~cm} \mathrm{~d}\end{array}$ & 0.2 & N/A \\
\hline
\end{tabular}

a Full Network Names:

Red Amazónica de Inventarios Forestales (RAINFOR)

Dinámica y crecimiento del Bosque Tropical Venezolano (DBTV)

Tropical Biomes in Transition (TROBIT)

African Tropical Rainforest Observation Network (AfriTRON)

Andes Biodiversity and Ecosystem Research Group (ABERG)

Tropical Forests in the Changing Earth System (T-FORCES)

Red Colombiana de Monitoreo de los Bosques (COL-TREE)

Global Ecosystems Monitoring (GEM)

Programa Ecológico de Longa Duração (PELD-TRAN)

Amazon Tree Diversity Network (ATDN)

Programa de Pesquisa em Biodiversidade (PPBio) 
Programa de Pesquisas em Caracterização, Conservação e Uso Sustentável da Biodiversidade (BIOTA)

Fire-Associated Transient Emissions (FATE)

Rede Amazônia Sustentável (RAS)

Monitoreo de las Areas Naturales Protegidos del Peru (MonANPeru)

Projeto Nordeste (Nordeste)

A Socio-Ecological Observatory for Southern African Woodlands (SEOSAW)

Red de Investigación y Monitoreo del Bosque Seco Tropical en Colombia (Red BST-Col)

Carnegie Airborne Observatory (CAO)

Red Subtropical de Parcelas Permanentes (RedSPP)

Red de Bosques Andinos (RBA)

Tree Diversity, Composition and Carbon in Andean Montane Forests (sANDES)

African tropical Montane forest network (AfriMont)

Red de parcelas Gentry (RedGentry)

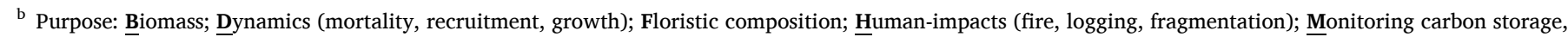

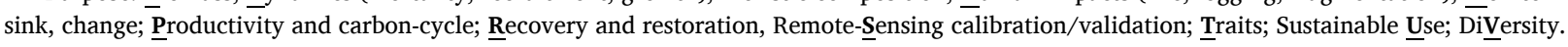

${ }^{\mathrm{c}}$ With nested sub-plots for smaller stems.

In Africa, our early networking focussed on assessing whether there were similar patterns of changes in carbon stocks as observed in South American forests and the causes of such changes. Efforts began in 2001 to recensus many of the earlier plots installed in post-independence Africa (UK funding to O. Phillips, Y. Malhi and S. Lewis), which were later formalised as the African Tropical Rainforest Observation Network (AfriTRON; Lewis et al., 2009) and catalysed a tripling of the African multi-census plot dataset over the last decade (Hubau et al., 2020). These span 12 African countries with moist forests from Sierra Leone in the west to Tanzania in the east. Like RAINFOR in Amazonia, AfriTRON pools expertise and data to tackle long-term, large-scale questions relating to the ecology and biogeochemistry of tropical forests. Networks sharing a similar ethos with programmes in Africa now include TEAM, DynAfFor (Gourlet-Fleury et al., 2013), TmFO and ForestGEO. Recently, the SEOSAW (SEOSAW partnership, 2020) and AfriMont networks have also been established, extending long-term plots into the extensive southern woodlands and savannas and Africa's distinctive montane forests.

Our work in Southeast Asia began in 2001 to assess forest carbon balance and later developed into a network once Lan Qie undertook fieldwork and networking. European Research Council investment (TFORCES 2012 grant to Phillips, Malhi and Lewis) enabled intensive

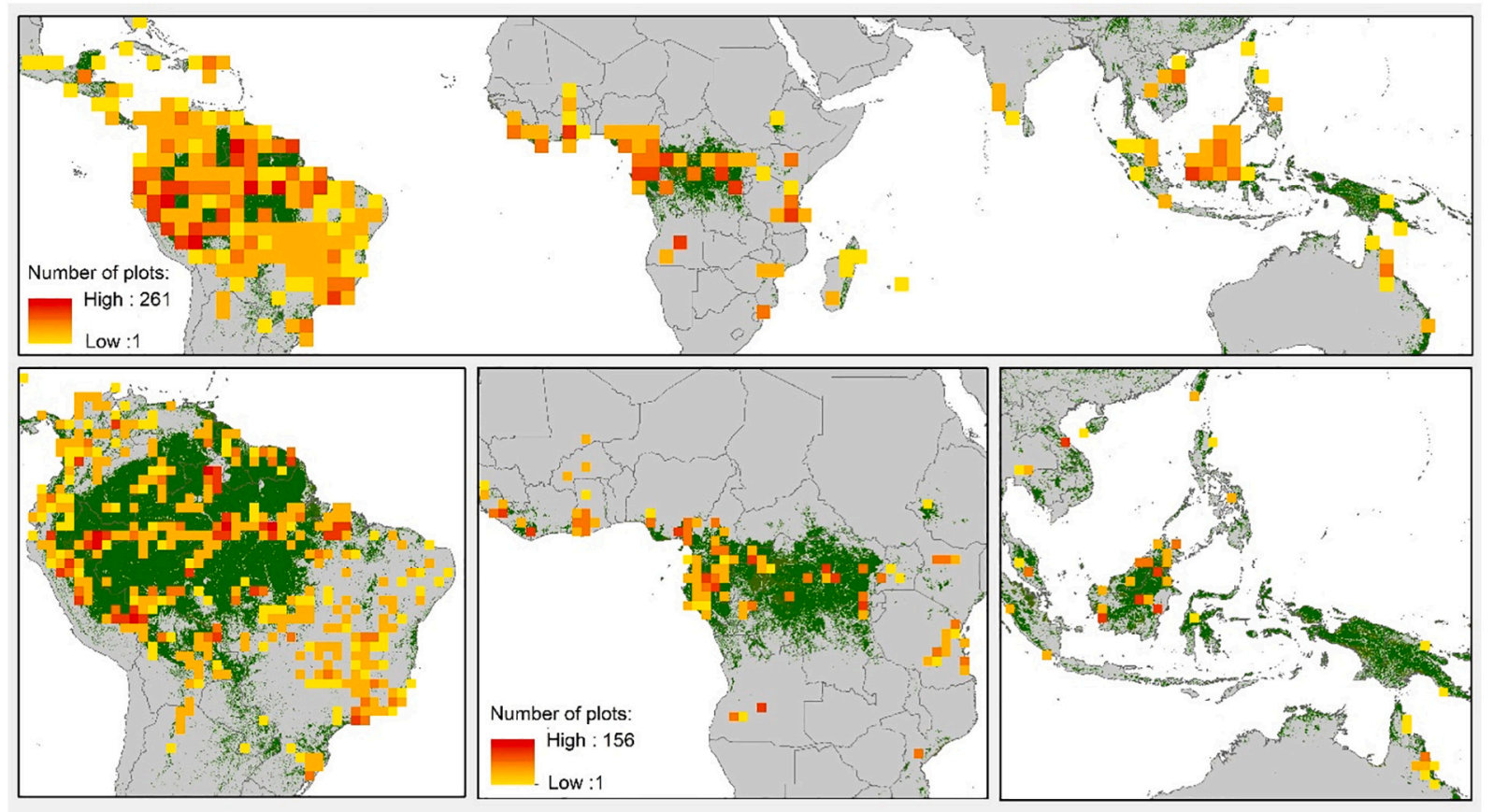

Fig. 1. Current extent of ForestPlots.net.

Top: Pantropical plot sampling density per 2.5 degree square with the 4062 multiple- and single-inventory plots hosted at ForestPlots.net. These plots contribute to 24 networks including RAINFOR, AfriTRON, T-FORCES, ATDN, BIOTA, COL-TREE, FATE, GEM, Nordeste, PELD, PPBio, RAS, RBA and SEOSAW. Forest cover based on the Global Land Cover 2000 database (JRC, 2003) with tree cover categories: broad-leaved evergreen; mixed leaf type; and regularly flooded. Our plots also extend into neotropical and African savannas; Bottom: The same plot sampling but displayed at higher-resolution (1-degree grid cells) for each focal continent, South America, Africa, and Southeast Asia and Australia. 
campaigns to develop long-term plot networking in Borneo (Qie et al., 2017), and supported African recensuses (Hubau et al., 2020). While smaller than its Amazonian and African counterparts, the Asian network builds on plots installed by a number of foresters and botanists as long as 60 years ago. Critically, RAINFOR, AfriTRON, T-FORCES and TmFO use the same field and analytical protocols.

How can we combine the different strengths of these and other initiatives to maximise their impact on science and society? To achieve this requires shared data management tools and horizontal organisational structures that foster leadership by tropical scientists. Our plot data management scheme was originally conceived in 2000 as a desktop database to support RAINFOR analyses of spatial variation in wood density, biomass, productivity, and changes in biomass over time (Baker et al., 2004a, 2004b; Malhi et al., 2004). This was expanded to draw together inventory data from $>100$ sites in Amazonia and then African forest plots to include some of the longest running monitoring sites worldwide (Peacock et al., 2007).

Since 2009 we have developed a Structured Query Language web application with sophisticated programming, providing a one-stop platform to a growing global community of contributors and users (López-Gonzalez et al., 2011). Now, ForestPlots.net supplies ecological informatics to colleagues in scientist-led networks from 54 countries working across 44 tropical nations (Fig. 1). Key advances in this platform include the ability to manage complex time-series data, track species linked to high-quality botanical records, and analyse records with common BiomasaFP R-language protocols (López-Gonzalez et al., 2015). While focussed on species identity, tree growth, mortality and carbon dynamics, ForestPlots.net encompasses many related forest attributes including lianas, soils, and plant traits.

At their heart, long-term plots are an intensely human enterprise and so we also document the personal contributions to plot establishment and continued monitoring. By tracking who did what, and when, we also

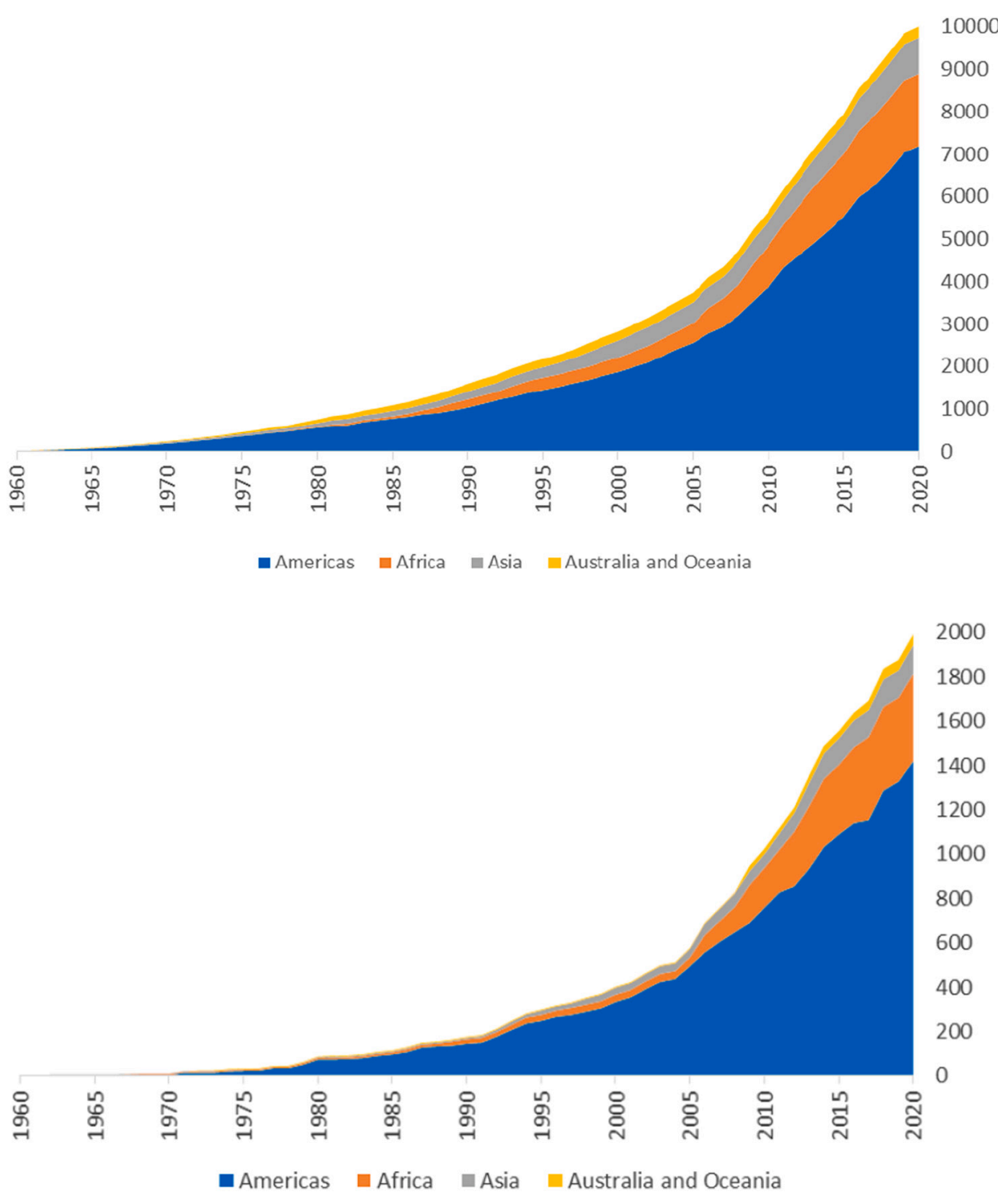

Fig. 2. Growth of pan-tropical forest monitoring since the mid-twentieth-century.

Top: Plot-censuses curated at ForestPlots.net by date of census.

Bottom: Cumulative number of contributors to ForestPlots.net by date of first recorded fieldwork. Growth was slow following the first census in 1939, only reaching 100 censuses by 1969 . For early censuses, records of field team personnel and leaders are often sparse or absent. Note that 'contributors' are defined inclusively to reflect members of indigenous communities, protected area guards, parataxonomists, students, and technicians, as well as principal investigators, botanists, and other specialists. 

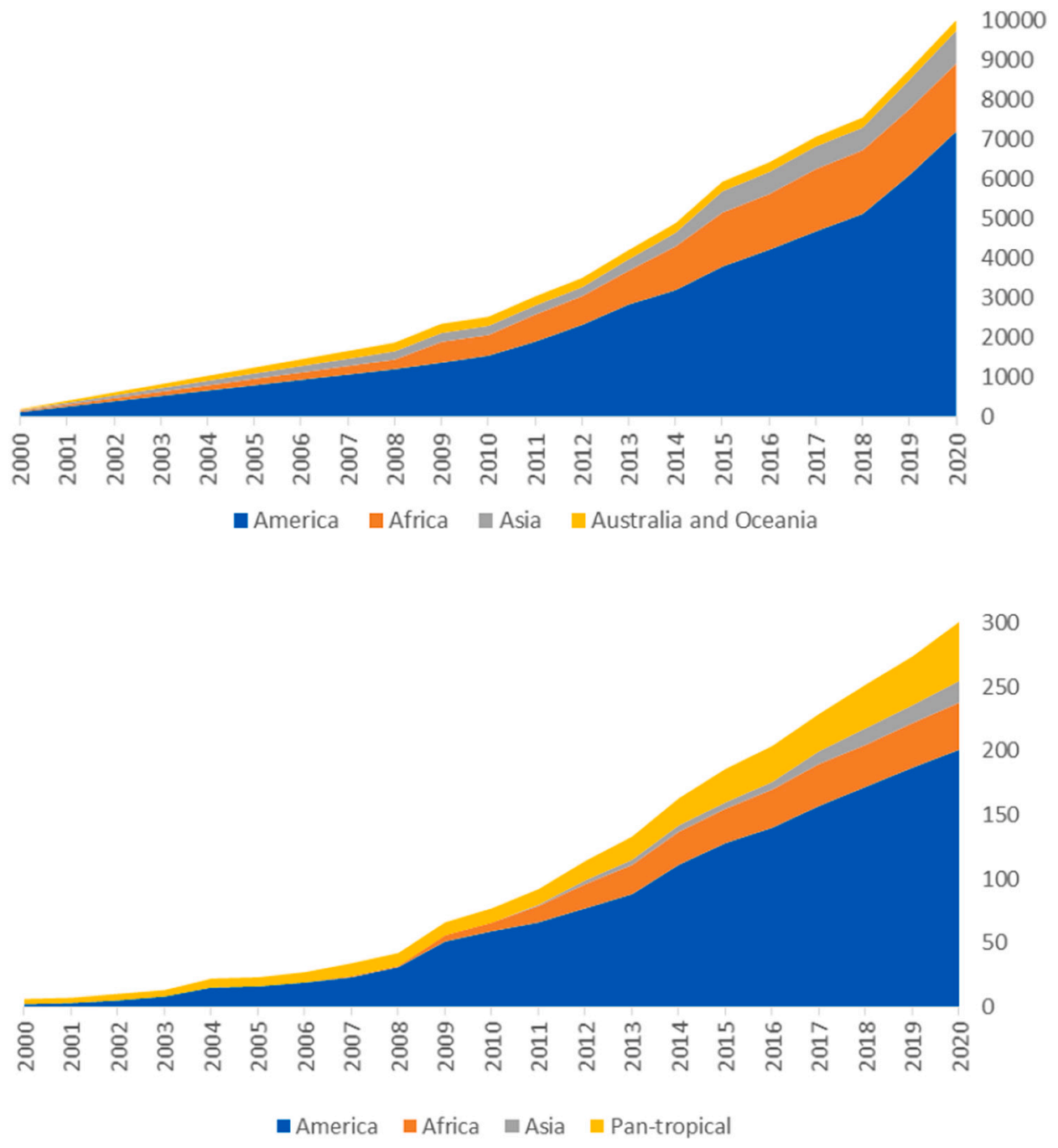

Fig. 3. Growth of ForestPlots.net and its contributing networks since 2000.

Top: Cumulative upload of unique plot censuses to ForestPlots.net by date of upload (pre-2009 uploads to pre-internet versions allocated evenly back to network beginnings);

Bottom: Cumulative peer-reviewed scientific articles based on network plots, excluding research based on single-plot studies. honour the inter-generational aspect of plots that allows modern analysts to stand on the shoulders of giants. With ForestPlots.net data contributors retain control and are able to manage, share and analyse their records using a common toolset. If new projects requesting to use their data are proposed they can agree to collaborate, or not, as they wish. Contributors often propose their own multi-site projects. ForestPlots.net can provide DOIs to datasets, further ensuring that contributors are properly acknowledged. Developing this functionality has supported a surge in multi-site and multi-national analyses that are increasingly initiated by scientists from the tropics, gradually supplanting the traditional model where researchers from the Global North lead. In sum, ForestPlots.net enables the level of control and collaboration that individual researchers wish for while also promoting network and multi-network integration. In turn, this is empowering data owners and networks and helping to transform the face of tropical ecological science.

The networks and ForestPlots share a 20-year history, but as we have seen the history of plot monitoring is much longer. The first recorded census in ForestPlots.net dates from 1939 in Budongo, Uganda. Forty years later, 676 censuses had been completed from 90 plots, but since 1979 fieldwork has accelerated greatly with $>10,000$ censuses completed across 4000 plots by 2020 (Fig. 2a). This acceleration is reflected by the growing community of contributors, which by 2020 had reached 2000 individuals (Fig. 2b). ForestPlots.net itself has grown steadily both in terms of censuses uploaded and in outputs (Fig. 3). The neotropics dominate much of this inventory and monitoring effort as well as the growth of ForestPlots.net in particular, but contributions from Africa and other continents are increasing (Figs. 2, 3). Scientific outputs emerging from this collective effort have always spanned local to global scales but now have an increasingly pan-tropical theme (Fig. 3b).

\section{Environmental representation}

While it is not possible to intensively sample the whole tropical forest extent, in practice RAINFOR, AfriTRON and T-FORCES have managed to cover almost the entire climatic and geographic space across the humid tropics with permanent plots (Fig. 4a) as well as extensively sample the 
1. Geographic distance $(\mathrm{km})$

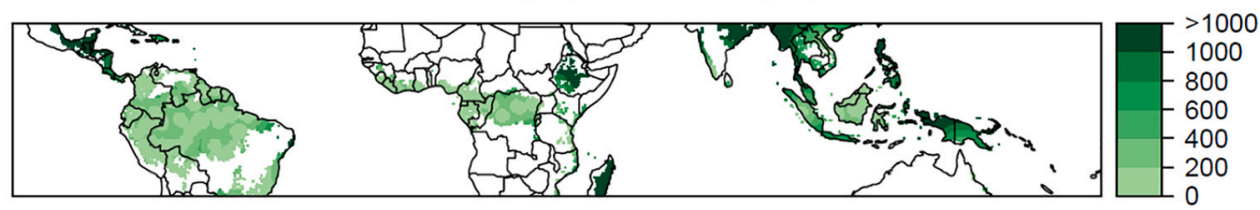

2. Environmental distance (Euclidean distance [SD])
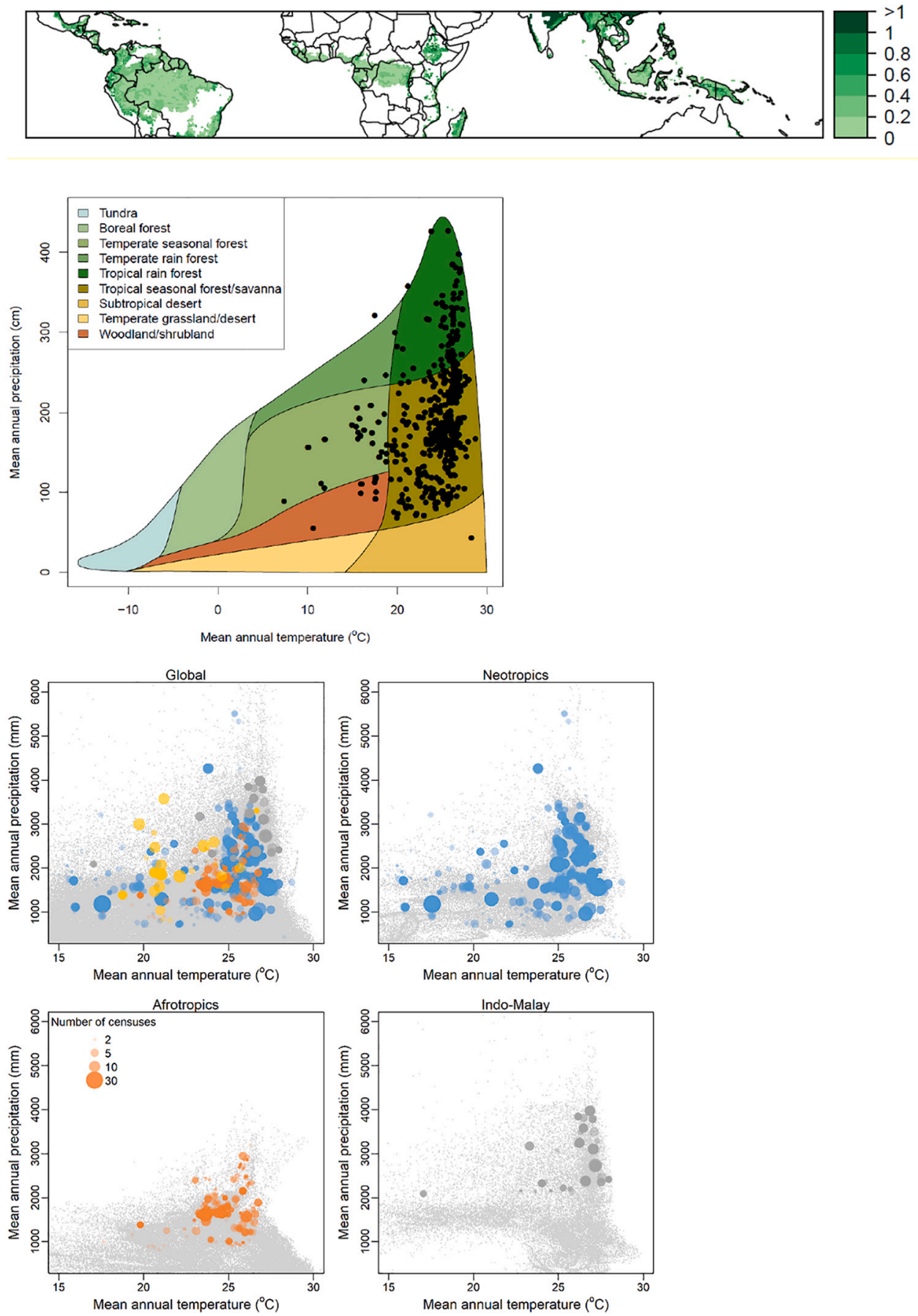

Fig. 4. Network coverage of geographical and climate space.

Analyses include $>1500$ permanent plots managed at ForestPlots.net. (a) Top panels: (1) Geographic distance between multicensus plots across the humid tropical forest biome; and (2) Minimum climate dissimilarity (Euclidean distance on variables scaled by their standard deviation, accounting for mean annual temperature, temperature seasonality, mean annual precipitation and precipitation seasonality), where for each cell environmental distance represents how dissimilar a location is to the most climatically similar plot in the network. Note that some poorly sampled areas are mostly deforested, such as Central America, Madagascar, and much of tropical South and Southeast Asia. The baseline map depicts WWF terrestrial ecoregions (Olson et al., 2001). (b) Middle panel: Tropical plots displayed in global biome space (Whittaker diagram), showing the main concentration of plots from lowland wet through to moist forests and savanna, with some samples in cooler montane climates. (c) Lower panels: Plots displayed within tropical humid and sub-humid climate space, with plots displayed colour-coded by continent (see Fig. 2) and symbol size corresponding to total census effort. Note the important differences in baseline climatic conditions between continents. 
biome space of the terrestrial tropics except for semi-arid biomes (Fig. 4b). Within each continent coverage has been focused on the moist tropical lowlands with sampling extending into montane and drier forest systems most effectively in South America (Fig. 4c). Plots also cover the complex edaphic variation present in Amazonia (Quesada et al., 2012) where they encompass landscape-level variability within old-growth forests (Anderson et al., 2009, 2010). This effective representation of structurally intact moist forests provides good support for large-scale inferences from what is, inevitably, a limited sample of the domain. It is important to note that many tropical countries lack statistical inventories of forests, let alone long-term monitoring or historical baselines, so research plots fill critical gaps in global and national observations.

Yet significant work remains to increase representativeness, better understand impacts of geological and edaphic variation, and expand sampling in remote areas especially in parts of Amazonia, the central Congo Basin, and New Guinea (c.f. Brearley et al., 2019, Fig. 4 below). Fuller environmental coverage can help networks address challenges such as monitoring of protected area effectiveness (Baker et al., 2020) and providing calibration-validation of Earth Observation space-borne sensors (Chave et al., 2019). Beyond the lowland humid tropics, special effort is also needed for long-term, ground-based monitoring in particular environments. Expansion is especially required for: (i) secondary forests and those impacted by disturbance events such as logging, fragmentation, and wildfires (e.g. Chazdon et al., 2016; Elias et al., 2020; Villela et al., 2006); (ii) montane forests, which harbour exceptional concentrations of endemism and are at great risk of biodiversity loss due to deforestation and climate change and therefore represent urgent conservation opportunities (e.g. Malizia et al., 2020); (iii) Asian dry forests, and (iv) the wider extent of tropical dry forest and savanna biomes, which are home to distinctive biotas and significant carbon stocks of their own (DRYFLOR, 2016; Norden et al., 2020; Pennington et al., 2018). ForestPlots.net partner groups are expanding research and monitoring in such critical areas beyond the structurally intact lowland forests that have been the main focus of RAINFOR and AfriTRON.

\section{Discovery: forest ecology across the tropical continents}

RAINFOR, AfriTRON and T-FORCES plots have generated ecological and biogeographical insights that have only been achievable via largescale collaboration. RAINFOR has revealed that Amazonian forests differ substantially from one another, even those that share essentially identical climates. For example, basal-area weighted wood density of northeastern forests is $50 \%$ greater than that of southern and western forests. This reflects floristic differences (Baker et al., 2004a, 2009; Fyllas et al., 2009; ter Steege et al., 2006; Honorio Coronado et al., 2009; Patiño et al., 2009), which, in turn, are associated with large differences in forest dynamics. Stem turnover is twice as fast in the west and south as the east (Phillips et al., 2004) due to younger soils with poorer structure providing less rooting support (Quesada et al., 2012; Schietti et al., 2016) and in spite of only modest productivity differences (Malhi et al., 2004, 2014a). In contrast, biomass in north-eastern Amazonia is higher than elsewhere due to the reduced mortality risk and hence bigger trees and denser wood (Baker et al., 2004a, Malhi et al., 2006, Marimon et al., 2014, Pallqui et al., 2014, Johnson et al., 2016, Alvarez-Davila et al., 2017, Phillips et al., 2019).

In Africa, AfriTRON plots also show that species-driven differences in wood density prevail at large scales. In mature forests, soil-related compositional differences cause significant variation in basal-area weighted wood density. Forests on younger and more fertile acrisols and cambisols have 10 and $20 \%$ lighter wood than those on arenosols and histosols, respectively (Lewis et al., 2013). Similarly to Amazonia, African forests growing on older, less fertile soils have higher biomass (Lewis et al., 2013). Local and regional variation in soils and forest attributes are important within both continents but the key difference is that only Amazonia has clear continental-scale gradients in wood density, due to the powerful influence of Andean orogeny in the west. This leads to young, geologically dynamic landscapes with fertile, lessdeveloped soils, influencing speciation, immigration and extinction, and contrasts with the ancient, stable Brazilian and Guianan Shields of the east.

Large-scale analysis thus reveals how soils and species help control the carbon that tropical forests store. This has implications for monitoring carbon stocks using remotely-sensed data. In tropical forests neither soil nor tree composition is easily perceived from space. For example, RAINFOR plots show that LiDAR-derived biomass estimates of Amazonian forests are problematic because they do not perceive the critical large-scale floristic gradients (Mitchard et al., 2014). Accounting for such limitations by relating plot-derived woody density and allometry to LiDAR sampling shows that plots greatly improve biomass maps (Mitchard et al., 2014; Avitabile et al., 2016). Thus the role of soils and species composition in affecting biomass carbon is a key reason why ground data are essential for mapping forests (Chave et al., 2019). While Earth Observation has huge benefits in terms of spatial coverage and frequent updates, the incorporation of plot-derived compositional data greatly improves our understanding of carbon storage patterns over large scales.

When networks using the same protocols are combined it is also possible to discover and explore variation between continents too. Common protocols have revealed major pan-tropical variation in vertical structure, including tree height and height-diameter allometry (Feldpausch et al., 2011) which have impacts on biomass (Banin et al., 2012; Feldpausch et al., 2012; Sullivan et al., 2018). African forests average one-third higher biomass per unit area than Amazon forests (Lewis et al., 2013), yet have roughly one-third fewer stems $>10 \mathrm{~cm}$ diameter per unit area. This may be driven by systematically lower tree mortality in these forests (Hubau et al., 2020; Sullivan et al., 2020). Similarly, comparing climatically and edaphically similar forests in parts of Borneo with northwest Amazonia reveals that Bornean forests produce much more wood, with trees growing up to $50 \%$ more rapidly than those of Amazonia. This suggests that differences in phylogenetic

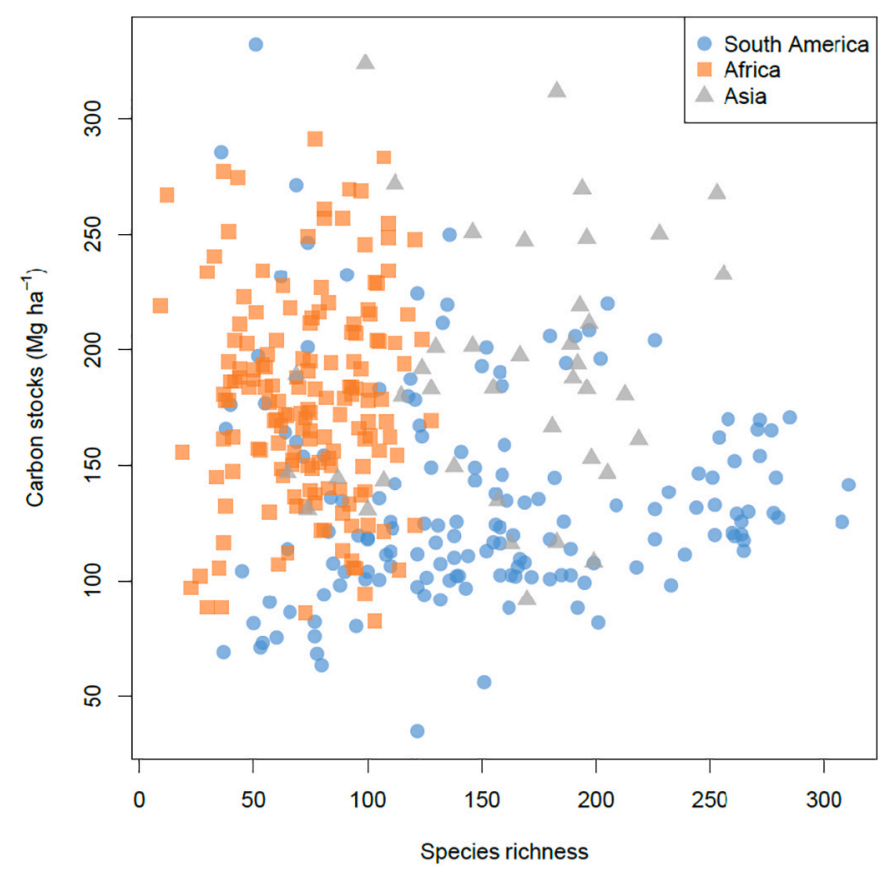

Fig. 5. Pantropical forest carbon storage is independent of species richness. There are no clear within-continent or pantropical relationships between carbon stocks and tree species richness per hectare in structurally intact oldgrowth tropical forests.

Figure adapted from Sullivan et al. (2017). 
composition of tree communities, especially the dominance of the dipterocarp family in tropical Asia (Corlett and Primack, 2011), determine the efficiency with which atmospheric carbon is converted to woody carbon (Banin et al., 2014).

Tree species composition and dominance strongly control forest function within continents too. For example, a recent RAINFOR study discovered that Amazon woody productivity is enhanced in more phylogenetically diverse forests (Coelho de Souza et al., 2019). Yet while Amazonian forests are very diverse, remarkably few species dominate in terms of stems (ter Steege et al., 2013, research led by the ATDN network), while biomass stocks and woody productivity are dominated by a different set of species (Fauset et al., 2015, RAINFOR network). Evidence also suggests that some of these 'hyperdominants' may have been long favoured by indigenous people as part of wider human influences on old-growth Amazon forests (Levis et al., 2017; Oliveira et al., 2020). These and other studies show that identity matters. Dominant species and their evolutionary history thus affect forest ecology and forest values, whether in terms of storing carbon, converting solar energy into wood or sustaining whole cultures.

These insights show that two of the defining challenges of the twenty-first century, climate change and biodiversity loss, are closely linked. How then do we best devise conservation strategies to achieve the targets of biodiversity protection and climate mitigation and adaptation? Can we rely for example on carbon conservation via schemes like REDD + to protect tropical diversity too? The answers to these questions depend on the relationship between diversity and carbon storage, but assessing this has been challenging due to the scarcity of inventories in which both carbon stocks and species identifications have been reliably quantified. By combining RAINFOR, AfriTRON and T-FORCES plots we found that for tropical trees diversity-carbon storage relationships barely exist at all (Sullivan et al., 2017, Fig. 5). For example, South America, the continent with the richest forests, actually stores the least carbon per hectare, while within continents there is no association. Independent data from the RAS network support this, showing that strong carbon-biodiversity relationships are only found in disturbed and secondary forests but not old-growth (Ferreira et al., 2018). As mature forests exhibit all possible combinations of tree diversity and carbon stocks it is clear that both need to be explicitly considered to protect the climate and biodiversity. In addition, long-term carbon storage is threatened by defaunation of large-bodied frugivores, often essential for dispersing large-seeded, heavy-wooded tree species (Peres et al., 2016). We cannot simply focus on carbon and achieve biodiversity conservation, and vice versa.

When network data are combined surprisingly large and coherent continental-level differences emerge (Fig. 6). African forests are remarkably species-poor at the 1-ha scale whereas South American and Asian forests are more than twice as rich on average, but also vary much more in species richness and diversity. The very richest forests in the world are located in parts of Western Amazonia, vindicating a claim by Gentry (Gentry, 1988a, 1988b) from more than three decades ago. African forests have many fewer stems than their Asian and South American counterparts, but South American forests have considerably less biomass. In terms of carbon gains Borneo's forests are outliers, being up to twice as productive as other forests. Yet it is in South America where woody carbon turns over fastest. Almost half the carbon in neotropical trees has been replaced since 1970 .

Overall these comparisons reveal remarkable differences between the tropical forest continents that are not strongly driven by rainfall,
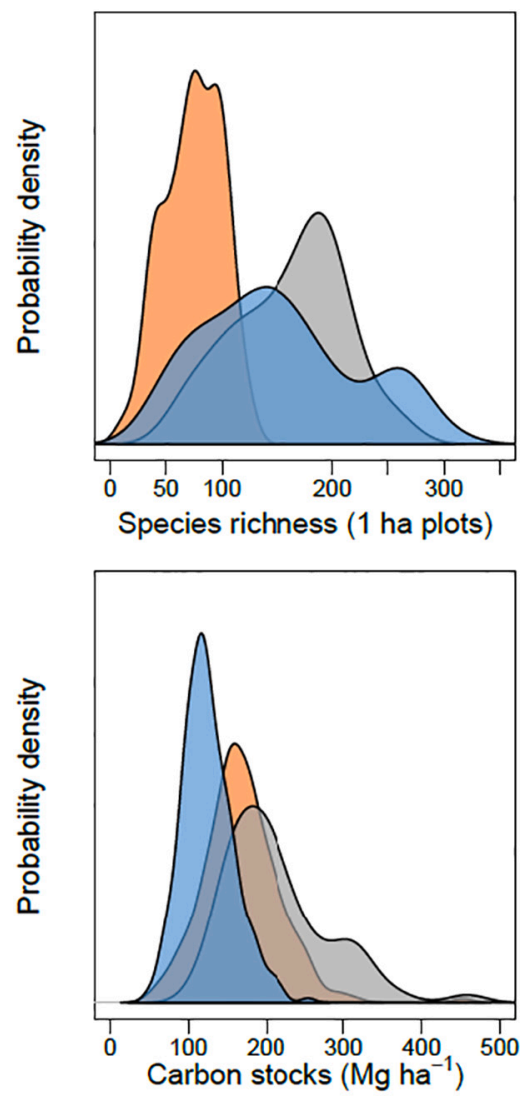
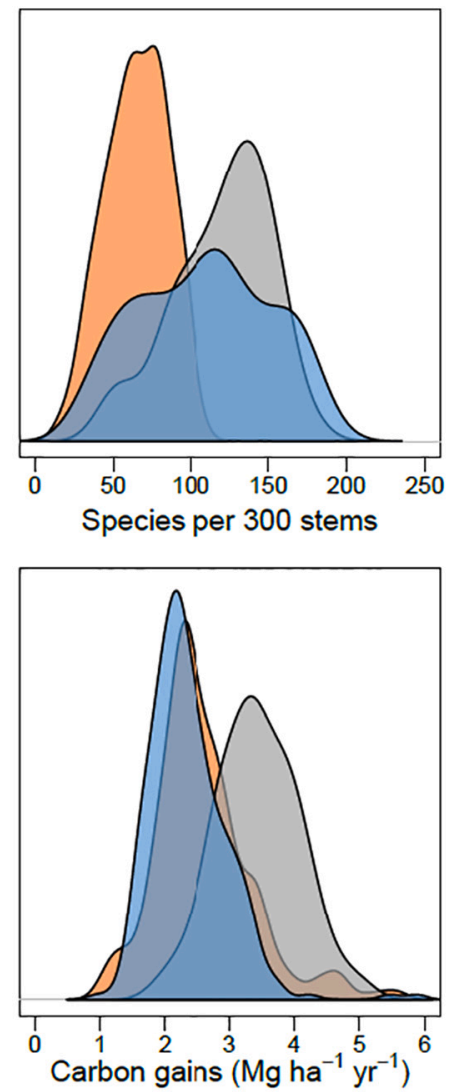
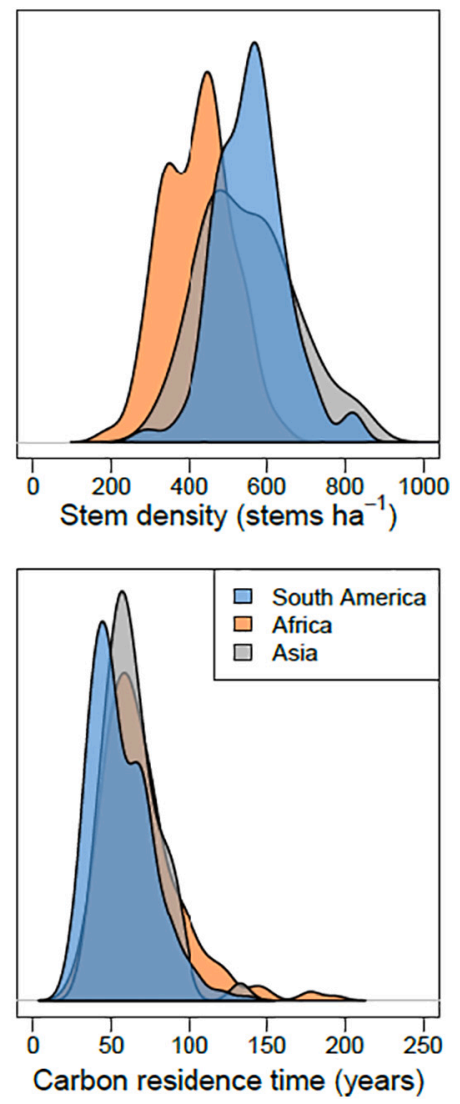

Fig. 6. Tropical continental macroecology.

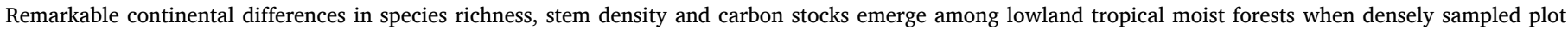

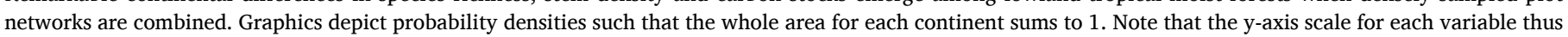

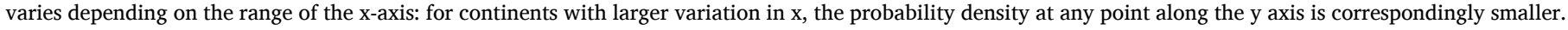
Analysis adapted from Sullivan et al. (2017, 2020). 
temperature or soil (Sullivan et al., 2020). The implication is that other factors related to the evolutionary and historical happenstance of each continent matter. We draw three higher level conclusions from this. First, global-scale ecological modelling ignores biological composition at its peril. Second, if there was ever any doubt, each continent clearly needs its own robust research and monitoring programme. And third, each region likely responds to climate change in its own, idiosyncratic way.

\section{Discovery: tropical forest change}

The single most significant scientific impact of these multiple permanent plot networks has been to transform our understanding of how tropical forests function in the Earth system.

As the most diverse and carbon-rich tropical biome, the fate of humid tropical forests will impact the future of all life on Earth. Until quite recently it was axiomatic that old-growth tropical forests are at equilibrium when considered over sufficiently large scales, and that any changes observed at smaller scales are driven by natural disturbancerecovery processes. However, large-scale imbalances observed in the global carbon balance have cast doubt on this assumption (e.g. Taylor and Lloyd, 1992). Over time, network analyses have helped to recast our understanding of contemporary old-growth tropical forests as being non-stationary systems. Their carbon, biodiversity and ecosystem processes are now widely recognised as dynamic and continually responsive to multiple anthropogenic drivers (e.g. Lewis et al., 2004b; Pan et al., 2011; Malhi et al., 2014b; Levis et al., 2017; McDowell et al., 2018; Reis et al., 2018). Key discoveries at this intersection between global change science and forest ecology and biodiversity include:

(1) A pantropical increase in tree turnover rates, representing the first evidence for a widespread impact of global anthropogenic change on old-growth tropical forests (Phillips and Gentry, 1994). The finding that these forests were changing was controversial at the time - let alone the inference that global drivers were responsible - and contradicted established ecological orthodoxy. The debate that ensued helped generate new questions and analyses (e.g. Sheil, 1996; Phillips, 1996; Phillips and Sheil, 1997) and address potential biases (e.g. Sheil, 1995, Condit, 1997, Lewis et al., 2004a, Gloor et al., 2009, Espirito-Santo 2014, Kohyama et al., 2019). A quarter of a century of research since then has rejected the notion that 'intact' tropical forests are unaffected by atmospheric changes and reinforced the central concept that all tropical forests are being influenced by a suite of large-scale contemporary anthropogenic drivers.

(2) Biomass dynamics have also accelerated in Amazonia. In parallel with the increases in stem dynamics, as RAINFOR grew it became clear that carbon fluxes via biomass growth and mortality were also increasing. Moreover, the increased gains in stems (recruitment) and biomass (woody productivity) clearly preceded increases in stem and biomass losses (mortality) (Lewis et al., 2004b, Phillips et al., 2004, 2008, Brienen et al., 2015, Nogueira et al., 2019). The mechanism underlying this acceleration of forest dynamics must therefore involve stimulated productivity via increased resources for plant growth, rather than direct stimulation of tree mortality such as by drought (Lewis et al., 2004).

(3) The Amazon forest carbon sink. In conjunction with faster growth and turnover, the biomass density of Amazonian forests has increased (Phillips et al., 1998; Baker et al., 2004b; Pan et al., 2011). Old-growth Amazonian forests have absorbed (net) atmospheric carbon for at least three decades now (Brienen et al., 2015), providing a true "subsidy from nature" with flux magnitude matching or exceeding net losses from neotropical deforestation (Aragao et al., 2014; Gatti et al., 2014). Thus, monitoring networks have shown that most Amazonian nations are on balance not net emitters of carbon (Espírito-Santo et al., 2014;
Phillips and Brienen, 2017). The location, magnitude and persistence of this old-growth carbon sink has important implications for guiding approaches to meeting nationally differentiated targets for controlling climate change (Vicuña Miñano et al., 2018).

(4) The African forest carbon sink. The AfriTRON network discovered a long-term net biomass increase similar in magnitude to that of the Amazon in the 1990s and early 2000s (Lewis et al., 2009). The consistency of these results on a second continent supports the idea that global drivers of change can affect even the most remote forests. The fact that biomass is increasing across the entire wood density spectrum of tree species implies that forests are responding to increasing atmospheric $\mathrm{CO}_{2}$ concentrations (Lewis et al., 2009). The long-term increase in carbon stocks of African forests was recently updated and confirmed, with three times as many plots showing continued sink strength (Hubau et al., 2020).

(5) The Pan-Tropical forest carbon sink. Once the T-FORCES network allowed sufficient plot coverage across remaining Bornean forest, a similar increase in aboveground biomass over recent decades was revealed (Qie et al., 2017). Thus the three continental networks discovered that old-growth tropical forests as a whole have functioned as a long-term sink. Our ground measurements revealed that more than one billion tonnes of carbon were sequestered by tropical forests each year over the 1990 s and early 2000s, i.e. half the terrestrial global carbon sink (Pan et al., 2011) and sufficient to significantly slow climate change. The fact that the main blocs of remaining tropical forests are en masse out-of-equilibrium and undergoing biomass increases of similar magnitude implies a common global driver of growth. Increasing atmospheric $\mathrm{CO}_{2}$ is the most parsimonious candidate and is consistent with predictions from first principles (e.g., Phillips and Gentry, 1994, Huntingford et al., 2013), inference from $\mathrm{CO}_{2}$ fertilization experiments (Terrer et al., 2019), analyses of the global carbon budget (Ballantyne et al., 2012; Gaubert et al., 2019), observed greening of forests unaffected by land-use change (Piao et al., 2019), and recent plot analyses showing a significant role of $\mathrm{CO}_{2}$ (Hubau et al., 2020).

(6) The Amazon sink is slowing. After 30 years of monitoring Amazonian forests, the RAINFOR plots show that the rate of increase in forest growth is declining. Tree mortality rates have increased in some regions, leading to a slow decline in the magnitude of the net biomass accumulation (Brienen et al., 2015; Phillips and Brienen, 2017). The subsidy from nature provided by tropical forests may be time-limited.

(7) Recent droughts in Amazonia have had large impacts. Longterm plots monitored immediately before and soon after droughts reveal that these forests can switch rapidly from being a major sink to a source of carbon. Both the 2005 and 2010 Amazon droughts had a net impact on the order of $1 \mathrm{Pg}$ of carbon, driven primarily by drought-induced mortality (Phillips et al., 2009, Lewis et al., 2011; Doughty et al., 2015, Feldpausch et al., 2016). RAINFOR and GEM have quantified the drought sensitivity of the world's biggest rainforest and found that the key process affected was tree mortality rather than growth or photosynthesis. The impact on the biomass carbon sink of the 2010 drought and nondrought years matches independent inferences from measurements of atmospheric $\left[\mathrm{CO}_{2}\right]$ using aircraft (Gatti et al., 2014).

(8) The African and Amazon sinks have diverged. Thirty years of monitoring AfriTRON plots show that African forests have continued to function as a carbon sink, although the most intensively monitored plots suggest that the sink may be declining (Hubau et al., 2020). When analysed together with RAINFOR data, within-plot changes over time reveal a common set of drivers that suggest the sinks will decline, with African forests lagging behind Amazonian forests by 15-20 years (Hubau 
et al., 2020). Changes across both continents are best explained by a combination of the positive effects of increasing $\mathrm{CO}_{2}$ in enhancing productivity and the negative effects of higher temperatures and droughts in suppressing growth and accelerating mortality, combined with the intrinsic properties of forests themselves. The time-lag of the African sink saturation is due to longer carbon residence times in African forests, so that mortality catches up more slowly than in forests with faster turnover. Amazonian forests are often harder hit because they are hotter and can be drought-prone (Hubau et al., 2020). Together, the pan-tropical plot networks have revealed long-term trends in carbon storage and determined which drivers matter, which processes are affected, where they are impacting, and what the lags are.

(9) The future of the tropical forest carbon sink. Monitoring the present and recent past of forest behaviour can also reveal likely future scenarios as the climate continues to change. Our plot networks provide two powerful and independent lines of evidence. First, the long-term sensitivity to climate emerges from a space-for-time analysis based on 813 plots across the Earth's tropical forests. This shows how maximum temperature and dry season intensity combine to determine the equilibrium climate controls on forest carbon, acting on productivity and mortality to limit forest carbon storage in the long-term (Sullivan et al., 2020). Forests exhibit remarkable thermal resilience under low amounts of warming, but in the hottest forests $\left(>32.2^{\circ} \mathrm{C}\right.$ max. temp.) biomass carbon drops off rapidly. Most of the biome will exceed this value with one further degree of warming (approximately equivalent to a $2{ }^{\circ} \mathrm{C}$ increase above pre-industrial levels). Second, analysing recent changes in productivity and mortality as a function of recent climates, and coupling them with future climate scenarios, confirms that the carbon sink is likely to decline (Hubau et al., 2020). A key uncertainty with these latter projections is the extent to which local resilience due to shallow water-tables (Sousa et al., 2020) may mitigate effects, and whether more compositional changes will extend the carbon sink further if species better-adapted to the new conditions compensate for others' losses. The analysis by Sullivan et al. (2020) confirms that lagged species-related resilience is likely as long as forests do not experience substantial warming.

(10) Tropical forest biodiversity is changing. RAINFOR data show that an entire group of plants, lianas (woody vines), are increasing in dominance across Amazonia (Phillips et al., 2002). Large lianas in turn contribute to higher tree mortality (Phillips et al., 2005). Tree community composition is changing too. In the Andes, plots of ABERG, RBA and RedSPP show 'thermophilization' - as communities become more warm-adapted (e.g Fadrique et al., 2018). Climate change is inducing large-scale change in tropical lowland trees too, as wet-adapted taxa in Amazonia face greater mortality risks from drought (Esquivel-Muelbert et al., 2017, 2019) while a shift towards drought-deciduous tree species is observed in west African plots experiencing a multi-decadal drought (Fauset et al., 2012, Aguirre-Gutiérrez et al., 2019, 2020). In both continents these community responses to drought coincided with biomass gains. Nonetheless, because of the long generation times of tropical trees the compositional change has not kept pace with the drying of Amazonia (Esquivel-Muelbert et al., 2019). This suggests that further community change is inevitable, even before accounting for losses driven by deforestation and disturbance of remaining forests (Barlow et al., 2016). Current models lack the capacity to account for variation in tropical woody plant biodiversity and demographic processes and their lagged responses to global change drivers.

In sum, highly distributed, long-term monitoring of the world's richest forests has profoundly increased our understanding of nature's sensitivity to climate change. It has shown that intact forests have been surprisingly resilient, but that many are now reaching the limits of their tolerance to global heating and drying. Looking forward, many of the key uncertainties that remain concern the responses of tropical biodiversity itself. This includes the extent to which the great biocomplexity of tropical forests themselves will provide an effective and timely insurance policy in the face of rapidly changing climates. To understand this, forest monitoring must continue.

\section{Challenges and the future of tropical forest monitoring}

Large-scale plot networks have not only made a series of crucial scientific discoveries and advances, but even more profoundly the Social Research Network model pioneered by RAINFOR since 2000 has influenced how the science itself is being done. Tropical ecology has undergone a remarkable shift from a small cadre of researchers working in one or two sites to a more globalized and decentralised process with greatly increased contributions from tropical scientists. This has been made possible by supporting highly-distributed researchers and field sites, establishing mechanisms for shared data management, fostering an equitable concept of data ownership, and embracing groups who are often marginalised in research. Importantly, the network model is nurtured by researchers placing trust in the sharing of hard-won data to answer big questions and recognising the value of developing trusting relationships over time. Finally, the growth of interactive multi-site, multi-cultural science has benefited hugely from standardized field and analytical methods that have been agreed upon, formalised and promoted. The ForestPlots.net experience demonstrates that collaborative, multi-polar structures help ensure breadth and resilience while supporting and encouraging the leaders of the future.

The transformative power of this approach has now led to the establishment of multiple plot-centred networks that are reshaping our understanding of tropical ecosystems. However, these networks face a number of key challenges to sustain the achievements made and enact even deeper transformational change, which we set out here.

\section{How can networks support leadership in the Global South?}

Although no single project can reverse the impact of centuries of global inequality, tackling the barriers to a more equitable world is the responsibility of all. Ecology and conservation science remain biased towards temperate ecosystems in terms of funding and topical focus (Di Marco et al., 2017; Reboredo Segovia et al., 2020), while tropical ecology is often detached from policy-making processes and most high-impact papers are still led from the North. Together with open data-sharing and long-term collaboration, more leadership of forest science from tropical countries helps to address these disparities and achieve more impact on forest and carbon management (e.g., Vargas et al., 2017; Baker et al., 2020). Supporting tropical students at different levels up to Ph.D. and mentoring beyond the doctoral degree is also important. To help, ForestPlots.net has made shared tools widely available, and especially data management analytic tools that support data contributors as much as users. To ensure fieldwork is valued and leadership in tropical researchers is fostered, we have developed a Code of Conduct to encourage contributions, support scientists in tropical countries, and promote mentoring of junior scientists. To oversee this we created a diverse steering committee that now supports dozens of projects each year (http://www.forestplots.net/en/join-forestplots/research-projects). As a result, the proportion of ForestPlots.net research projects and products led by tropical nationals has greatly increased, with $<10 \%$ of publications when RAINFOR began (2000-2004), rising to $35 \%$ in 2009 and $50 \%$ by 2019 . In spite of such gains diversifying leadership is a long-term process. Ultimately, sustained funding in and by tropical countries themselves will ensure they not only have strong training programmes to develop the core field and analytical skills that scientists need, but equal opportunities for career development. 

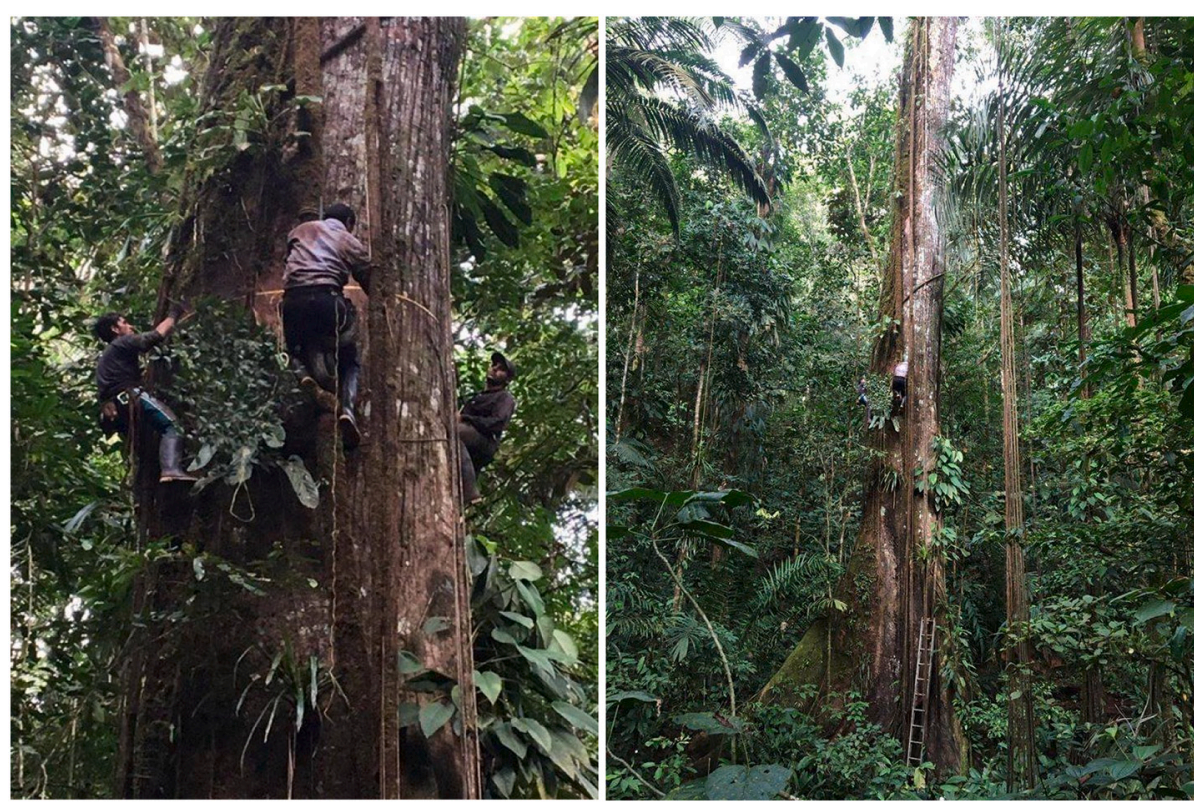

Fig. 7. Accurately measuring and identifying trees in remote tropical forests requires dedication, skill and courage.

To measure the diameter of this giant Ceiba (Malvaceae) tree in Reserva Amargal (Colombia's Chocó), three researchers of the COL-TREE network each needed to climb $>10 \mathrm{~m}$. Such techniques can be the most practical and accurate options for measuring large trees. Here, like many of our sites, there is no electric power, let alone a field station, and chronic insecurity due to political and social conflicts and narcotrafficking means that aircraft and laserscanners are not deployable. Images: Pauline Kindler, University of Rouen (France).

2. How should we value and recognise collaboration and leadership? Most of the obvious reward structures in science - job security, income, grant success, peer reputation and public acclaim - can favour a 'me first' approach. Credit accrues to individuals, but true collaboration involves trust, sharing and encouraging others. Collaboration is gratifying, but letting go of our egos can be challenging, while in larger groups there is greater risk that individuals feel their contributions go unnoticed. Likewise, the essential and major effort needed 'backstage' in ForestPlots.net to check data, update and develop data management, and support requests to utilize data, goes unseen. A partial developmental solution to this involves providing network contributors the opportunity to lead analyses with the expectation that these new leaders then support others with their analyses. Another approach is to reflect the diversity of contributions that underpin the success of networks by using a group author that shares credit among all, as in the current paper. These steps can promote the recognition of multiple contributions and development of tomorrow's leaders.

3. How do we properly value the long-term? Project and thesis timescales last from one to five years, but the lifespans of trees are measured in decades and centuries. What can seem vitally important in a hypothesis-driven research grant or a Ph.D. may, in fact, have little relevance to the longer natural rhythms of nature. What if the dominant processes governing climate responses of forests turn out to involve lifetime accumulated ecophysiological stress, tree demography and species migration? Clearly very long-term research is essential to decode these processes. Meanwhile, maintaining permanent plots is as much an expression of hope in the future as a stake in an immediate scientific outcome, as rewards may accrue to others distant in time and space. Indeed, we have all benefited from researchers installing plots from the 1930s onwards. These pioneers never dreamt that their careful tree measurements and botanical identifications would help reveal the impacts of climate change on tropical forests, but look what they have achieved! Long-term research programmes are simply irreplaceable, enabling us to discover, quantify, identify the causes of, and ultimately tackle environmental change.

4. Can we ensure fieldwork and human skills are valued for what they are? Technology provides many benefits to the scientific endeavor, but there are risks too, particularly in a field where long-term measurements may be perceived as unfashionable (Ríos-Saldaña et al., 2018). A serious risk is that the tail wags the dog: when technological advance is an end in itself, it is unlikely that scientific and human progress will follow. We should never forget the basic truth that human beings and their skills are essential to measure and identify tropical trees. It is notable that those measuring, climbing and collecting tropical trees in permanent plots are among the least wellpaid of all actors in the global scientific endeavor. Yet, these true key workers are irreplaceable as tree measurement in many locations is completely dependent on such labour and skill (Fig. 7) and, more broadly, combinations of people and technology provide the best results (next section). Moreover, because tropical tree floras usually run into the thousands of species (e.g., $>4700$ tree species in Peru, Vásquez et al., 2018), identification depends on the work of highly skilled climbers and botanists to collect material from canopies, make vouchers, and identify and permanently store them in herbaria. Without physical collections and the immense multicultural knowledge and skills that produce them, identifications are untestable hypotheses whose quality cannot be evaluated. But with vouchers, we have the names that are essential to test questions about diversity, composition, functional traits, and biomass.

5. How should we fund proven networks long-term? As the most pressing concern, this question intersects closely with all of the above. Few organisations have the vision to support long-term endeavours where leadership and credit is shared diffusely, many benefits accrue after decades, and where the most exciting discoveries may be unforeseeable. We recommend the following, potentially transformational changes to address the challenges and unlock the benefits of ambitious, longterm monitoring of tropical forests:

(i) Science Agencies have the foresight to build long-term research capacity and consciously adopt the challenge of international ecosystem monitoring and tropical career development;

(ii) Space Agencies, recognising that tropical fieldwork can measure the things they cannot and validate the attributes that they can, directly support the labour and skills of tropical forest scientists;

(iii) Development and Conservation Agencies who depend on a robust understanding of the long-term health of forests, recognise that high quality, long-term, on-the-ground monitoring of trees and the skills needed for this are vital for their agenda;

(iv) National and international climate adaptation and mitigation funders recognise that long-term scientific monitoring of mature forest carbon fluxes is essential for successful nature-based conservation and forest management, and to achieve 


\section{Box 1}

What does it take?

Clearly, long-term, ground-based monitoring of tropical forests requires a sustained global team effort. But just how much does it take to deliver tropical forest plot data in practice? It requires both skilled labour and funds. So here we address this question in terms of the human effort made thus far and the financial investment needed to monitor across continents.

(a) The Human Contribution: Network efforts include not only in-country field campaigns but much besides. To deliver from conception to product, high-quality data collected over many years and in dozens of countries requires multiple teams that are well-led and consistently trained in the proper protocols, quality control, and data management. In RAINFOR and AfriTRON this includes national or local field-team members to establish and remeasure plots, others to collect and identify plants and collect and analyse soils, colleagues to organize and manage the data, and others to sustain and lead the process nationally and globally - not to mention those who support these processes with essential administration, herbarium assistance, database development, analytical packages, information technology support, technical training and so on. Naturally some individuals contribute in several ways and roles change over time as lives change. All these local, national and global efforts ultimately depend on funding.

The average effort in the field, herbarium, and lab to install a typically remote and diverse 1-ha tropical forest plot and analyse its species and soil sums to 98 person-days, with an additional effort of 38 person-days to support and sustain these teams and data management. Together a total of 136 person-days are needed on average to deliver high-quality data from a new plot.

Recensusing a plot is usually less demanding (for example soil collection is not repeated and there are fewer plants to identify) but still considerable: 45 person-days in the field and herbarium, and 31 person-days to support and sustain the recensus. Therefore, 76 person-days are required to deliver high quality data from a recensused plot. These estimates represent long-term averages and are based on remeasuring plots within five years or less between each census, and assume the plot was installed using standard protocols. Naturally circumstances can vary from siteto-site and country-to-country.

Thus far our teams have established 4062 plots in tropical forests of which 1816 are recensused, from as little as once up to as many as 40 times each. The modal size of the 4062 plots is between 0.9 and 1.1 ha but there are smaller plots too ( 1844 are $\geq 0.9$ ha, and 2216 are $<0.9$ ha). The recensused plots tend to be larger: of the 1816 recensused plots, $62 \%$ are $\geq 0.9$ ha (1131) and $38 \%$ are $<0.9$ ha (675).

If we conservatively assume that plots $\geq 0.9$ ha (average size $=1.2$ ha) require 136 days to install and 76 days to recensus, and those $<0.9$ ha require half this effort (also likely to be conservative due to fixed costs for even the smallest plots), then the total effort to install these plots has been 196,248 person-days, and recensusing them has taken 357,940 person-days. In total this comes to 1518 years.

As if one remarkably talented and tireless individual had been working continuously since $502 \mathrm{CE}$.

(b) Cost of Sustained Continental Monitoring: How much does it cost to monitor Earth's remaining old-growth tropical forests with ground networks? This is a critical question given the exceptional ecological value of these systems, the threats they face, and the role they play in modifying the rate of global climate change.

At first sight this question appears difficult to answer, or to even agree upon the terms of reference. Scientists would ask and likely argue: Monitoring what? For whom? With what precision, level of confidence, or spatial and temporal resolution? Recognising such difficulties, we take a pragmatic approach and reframe the question. Instead we posit, How much will it cost to monitor tropical forests using all the permanent plots that have already been remeasured?

This question is tractable practically (these plots represent a known quantity: we know exactly where they are, what most of the species are, and to a large extent who can actually do the work - each of which is critical), it makes sense scientifically (the plots already have a baseline monitoring period against which we can assess any change, which is essential), and it is justifiable quantitatively (using somewhat smaller datasets than this we have already detected long-term changes in carbon balance, productivity and tree mortality on each continent, reported short-term changes in response to El Niño droughts and other climate anomalies, and attributed changes in carbon and biodiversity to climate drivers, all of which establish proof-of-concept). So here goes:

*There are 1105 remeasured ForestPlots.net plots in tropical forest South America $(422<0.9$ ha $+683 \geq 0.9$ ha $)$, 462 in tropical forest Africa $(109+353), 192$ in tropical forest Asia $(106+86)$ and 32 in tropical forest Australasia $(22+10)$. With all 1791 plots monitored on a four-year cycle this requires revisiting 448 plots annually, of which 165 are $<0.9$ ha and 283 are $\geq 0.9$ ha.

*Recensus costs can vary from site-to-site. Botanical identification is especially challenging in most of South America due to the extraordinary diversity, while some African forests are exceptionally remote. Employment, social security and health costs vary but are rising almost everywhere. On average, considering all the direct and indirect human effort required (above) and additional direct costs (including consumables, equipment, travel, subsistence, insurance, visas, permits, shipping, training, and IT), the current cost to deliver a high-quality tropical recensus is $\approx 18,000$ USD for plots $\geq 0.9$ ha, and at least half this for plots that are $<0.9$ ha. That's about 30 USD per tree.

[Installing plots is a costlier operation as it requires more expert time to collect and identify highly distributed trees. The total cost to properly install a high-quality tropical forest plot is $\approx 27,000$ USD for a 1 ha plot. When forests are recensused this start-up investment is leveraged as a contribution: this enables the subsequent monitoring of forest dynamics.]

Thus, the annual delivery cost for a pantropical, practical ground-based recensus programme capable of tracking and attributing forest change to published standards is estimated as:

$(28318,000+1659000) \approx 6.6$ million US dollars.

This annual investment is sufficient to ensure that ground-measurements track the biome-wide and continent-specific biomass carbon balance of the world's remaining tropical moist forests, as well as their climate sensitivity. It also provides ground calibration and validation for remote estimates of 
biomass. It further enables us to detect whether the tropical sink is now disappearing as predicted, and where and why, what the consequences for biodiversity are, and to determine how much intact ecosystems can contribute to countries' nationally determined contributions (NDCs) to climate mitigation.

While $\$ 6.6$ million is a significant sum it is instructive to compare it to funding required for other large-scale science initiatives. The United States alone spends $\$ 80$ million annually (i.e., twelve times as much) on its national forest inventory (Castillo and Alvarez, 2020). Space Agencies invest from ca. $\$ 80$ million to 500 million Euros for a single mission to estimate biomass from space for a few years (i.e, one to two orders of magnitude more). And as we have seen, ground networks ultimately not only transcend the short-term time windows of such missions but add huge value to them.

In conclusion, the ongoing cost of monitoring Earth's remaining tropical forests on the ground is extraordinarily small compared to the great scientific and practical benefits it provides. Meanwhile, tropical forests themselves are in greater trouble than ever before, even while providing tremendous and irreplaceable benefits to the people of the world. Now that the capacity to monitor tropical forests is established and proven, it is incumbent on all of us to ensure this collective effort continues and grows.

nationally determined contributions (NDCs) to reducing greenhouse gas emissions for decades to come.

Every one of these user groups requires successful networks with long-term, research-grade tropical forest plots to discern the status and change of biodiversity and to assess the stocks and flows of carbon.

\section{Achievements, impact and potential}

Despite the challenges, tropical forest science has come a very long way. Until recently, tropical ecology suffered from a massive data deficit. We had plenty of theory and conjecture, but few comparable observations over time and space to deductively put these ideas to the test or inductively generate new ones. Networks such as ForestGEO, RAINFOR, AfriTRON, and the wider ForestPlots community have contributed much to resolving this. By leveraging a remarkably old technology, forest plot networks have sparked a modern revolution in tropical forest science. They provide the means by which we have quantified the trajectory of tropical forest carbon balance, including its climate sensitivity, and now provide a Pan-Tropical Observatory for tracking these vital indicators of Earth's health going forward.

Permanent plots are now the prism through which ecologists address a rich suite of ecological questions, but they have also changed the way others see forests. For example, well-identified permanent plots have proved fertile ground for botanists to discover new tree species and genera (e.g. Reitsma, 1988, Baker et al., 2017, Wurdack and Farfan-Rios, 2017, Vásquez et al., 2018, Gosline et al., 2019, Vásquez and Soto Shareva, 2020), ethnoecologists to quantify forest people's values (Phillips and Gentry, 1993; Lawrence et al., 2005), atmospheric scientists to explore organic volatile production (Harley et al., 2004), ecophysiologists to assess why trees die (Rowland et al., 2015; McDowell et al., 2018), modelers to verify ecosystem simulations (Johnson et al., 2016), and foresters to predict and manage wood production and its impacts (Berry et al., 2008; Gourlet-Fleury et al., 2013). They provide critical infrastructure for whole-biodiversity and cross-taxa inventories, including exploration of cryptic canopy and soil faunal and microbial biodiversity (e.g., Nakamura et al., 2017). The impacts of these networks on policy are also growing. In Peru for example, ForestPlots.net, MonANPeru and RAINFOR have contributed to estimating National Forest Reference Emission Levels (NREF) since 2016, and our permanent plots are now being used to validate national contributions to the Paris Climate Accord via forest carbon sequestration (Vicuña Miñano et al., 2018; Baker et al., 2020). In Ghana, plots were needed to quantify historical and current carbon stocks, helping to establish baseline forest reference levels for the flagship Cocoa Forest REDD+ Programme (FCPF, 2017). In Gabon stratified-random sampling of high-quality AfriTRON plots is now used for the National Forest Inventory (Poulsen et al. 2020). Internationally, RAINFOR, AfriTRON, T-FORCES and 2ndFor provide the new IPCC default values for old-growth and secondary forest carbon sequestration to help countries develop their nationally determined contributions as part of the UNFCCC process (Requena Suarez et al.,
2019).

What of the future? As new technologies for probing forests become available, the highly distributed standardized long-term plots and networks of skilled tropical researchers represent critical infrastructure to enhance and calibrate new insights as they arise. The benefits of working within established plots go beyond simply having confidence in species identifications and hence biomass. By leveraging the wealth of information that permanent plots provide, we can increase the scientific value of new technology. For example, the ability to match individual trees from laser-scanning surveys to tagged, censused individuals provides critical information on growth and identity (Disney et al., 2018). Integrating long-term botanical and ecological records of plots with terrestrial and airborne laser-scanning in designated super-sites (Chave et al., 2019) can help overcome limitations of different approaches, providing greater certainty to biomass estimates (e.g., Schepaschenko et al., 2019). Hence forest networks can help unlock the value of spacebased efforts to monitor forests. Just as the constellation of Earthobserving environmental satellites is a public good, the constellation of forest plots provides highly complementary, critical global infrastructure. And last, but not least, as intact tropical ecosystems continue to shrink, burn and fray at the edges, permanent plots provide the indispensable baseline for understanding biodiversity and ecosystem processes too. They can be our shining North Star for guiding sorely needed restoration efforts throughout this century.

So far this effort has relied on the goodwill of highly distributed colleagues and dozens of grants from many sources (see Acknowledgments). Only long-term funding will ensure that the vital public benefits of plot networks continue to flow. Such support is surprisingly difficult to obtain (see Box 1). Yet twenty years of hard-won scientific results show that reliable and highly distributed monitoring is irreplaceable. They underscore the importance of welcoming all contributors to this effort, and of valuing the diverse skills needed to understand tropical biodiversity and its dynamics. Ultimately, we will understand the nature of tropical forests best when the science is global, local skills are fairly valued, and the development of tropical scientists is at its heart. Indeed, we know of no other model capable of achieving this.

\section{CRediT authorship contribution statement}

All authors have contributed to ForestPlots.net-associated networks by leading, collecting or supporting field data acquisition, or implementing and funding network development, data management, analyses and outputs. O.L.P. wrote the manuscript with initial contributions from S.L.L., M.J.S. contributed new analyses, M.J.S., G.L.P. and A.L. helped prepare the figures, and all authors reviewed the manuscript with many suggesting valuable edits. O.L.P., T.R.B., G.L.-G. and S.L.L. conceived ForestPlots.net. R.B., T.R.B., T.F., D.G., E.G., E.H., W.H., A.E.-M., A.L., S. L.L., K.M., Y.M., G.C.P., O.L.P., B.S-M., L.Q., and M.J.P.S have contributed tools, funding or management to its development since.

The article is attributed collectively as ForestPlots.net et al., with individual authors listed alphabetically first by country of institution 
and secondly by family name.

\section{Declaration of competing interest}

There is no conflict of interest.

\section{Acknowledgments}

This paper is a product of the RAINFOR, AfriTRON and T-FORCES networks and the many other partner networks in ForestPlots.net which support long-term forest science and monitoring across tropical countries. These initiatives have been supported by numerous people and grants since their inception. We are particularly indebted to more than one thousand four hundred field assistants for their essential help in establishing and maintaining the plots, as well as highly distributed rural communities and institutions. For additional assistance we thank Michel Baisie, Wemo Betian, Vincent Bezard, Mireille Breuer-Ndoundou Hockemba, Ezequiel Chavez, Douglas Daly, Armandu Daniels, Eduardo Hase, Muhammad Idhamsyah, Phillipe Jeanmart, Cisquet Keibou Opepa, Jeanette Kemp, Antonio Lima, Jon Lloyd, Mpanya Lukasu, Sam Moore, Klaus Scipal and Rodrigo Sierra. We thank Mark Burkitt for help developing the ForestPlots.net database. We acknowledge the long-term help provided by national and local government offices in all countries where colleagues work in facilitating the permission and documentation for fieldwork, as well as help provided by protected area and other authorities.

The networks have been supported by multiple grants, most notably the European Research Council (ERC Advanced Grant 291585 - 'TFORCES'), the Gordon and Betty Moore Foundation (\#1656 'RAINFOR', and \#5349 'MonANPeru'), the David and Lucile Packard Foundation, the European Union's Fifth, Sixth, and Seventh Framework Programme (EVK2-CT-1999-00023 - 'CARBONSINK-LBA', 283080 - 'GEOCARBON', 282664 - 'AMAZALERT'), the Natural Environment Research Council (NE/D005590/1 - 'TROBIT', NE/F005806/1 - 'AMAZONICA', 'PPFOR' E/M0022021/1), several NERC Urgency and New Investigators Grants, the NERC/State of São Paulo Research Foundation (FAPESP) consortium grants 'BIO-RED' (NE/N012542/1, 2012/51872-5), 'ECOFOR' (NE/K016431/1, 2012/51509-8), 'ARBOLES' (NE/S011811/1), 'SEOSAW' (NE/P008755/1), 'SECO' (NE/T01279X/1), 'NORDESTE' (NE/N012550/1, 2015/50488-5), the Royal Society (University Research Fellowships and Global Challenges Awards) ('FORAMA', ICA/ R1/180100), the National Geographic Society, the Centre for International Forestry (CIFOR), Gabon's National Parks Agency (ANPN), US National Science Foundation (DEB 1754647), and Colombia's Colciencias. We thank the National Council for Science and Technology Development of Brazil (CNPq) for support to the Cerrado/Amazonia Transition Long-Term Ecology Project (PELD/441244/2016-5), the PPBio Phytogeography of Amazonia/Cerrado Transition project (CNPq/ PPBio/457602/2012-0), the Goiás Research Foundation (FAPEG/PELD: 2017/10267000329), and several PVE and Productivity Grants. Funding for plots in the Udzungwa Mountains (Tanzania) was obtained from the Leverhulme Trust under the Valuing the Arc project. Plots in the Democratic Republic of Congo were funded by the Belgian Science Policy Office (SD/AR/01A/COBIMFO, BR/132/A1/AFRIFORD, BR/143/A3/ HERBAXYLAREDD, CongoFORCE), the Flemish Interuniversity Council VLIR-UOS (CD2018TEA459A103, FORMONCO II), and the European Union (REAFOR, FORETS projects). We acknowledge grant CEBA (ref. ANR-10-LABX-25-01) and the support of the Forestry Development Authority of Liberia. We also acknowledge the support of the European Space Agency. Data from RAINFOR, AfriTRON and T-FORCES are stored and curated at ForestPlots.net, a cyber-infrastructure initiative developed at the University of Leeds that unites permanent plot records and supports scientists from the world's tropical forests. The development of ForestPlots.net and curation of data has been funded by several grants including NE/B503384/1, NE/N012542/1 'BIO-RED', ERC Advanced Grant 291585 'T-FORCES', NE/F005806/1 'AMAZONICA', NERC New
Investigators Awards, NE/N004655/1, 'TREMOR', the Gordon and Betty Moore Foundation ('RAINFOR', 'MonANPeru'), ERC Starter Grant 758873 'TreeMort', EU Framework 6, a Royal Society University Research Fellowship, and a Leverhulme Trust Research Fellowship.

The manuscript has been developed with the encouragement of Richard Primack and Reinmar Seidler and has benefited from the constructive comments of three reviewers; we thank them all. Finally we thank our late, great colleagues whose unique contributions helped make possible all that the networks and ForestPlots.net have achieved together since the beginning: Samuel Almeida, Elisban Armas, José Armas, Sandra Brown, Kwaku Duah, Gloria Galeano, Alwyn Gentry, Max Gunther, Moïse Mikame, Norman Myers, Sandra Patiño, John Proctor, David Smith and Jean-Pierre Veillon.

\section{References}

Aguirre-Gutiérrez, J., Oliveras, I., Rifai, S., Fauset, S., Adu-Bredu, S., Affum-Baffoe, K. Baker, T.R., Feldpausch, T.R., et al., 2019. Drier tropical forests are susceptible to functional changes in response to a long-term drought. Ecology Letters 22, 855-865.

Aguirre-Gutiérrez, J., Malhi, Y., Lewis, S.L., Fauset, S., Adu-Bredu, S., Affum-Baffoe, K., Baker, T.R., Gvozdevaite, A., et al., 2020. Long-term droughts may drive drier tropical forests towards increased functional, taxonomic and phylogenetic homogeneity. Nature Communications 3346 (2020).

Alvarez-Davila, E., Cayuela, L., González-Caro, S., Aldana, A.M., Stevenson, P.R., Phillips, O., Cogollo, Á., Penuela, M.C., von Hildebrand, P., Jiménez, E., Melo, O., 2017. Forest biomass density across large climate gradients in northern South America is related to water availability but not with temperature. PloS One 12 (3).

Anderson, L.O., Malhi, Y., Ladle, R.J., Aragao, L.E.O., Shimabukuro, Y., Phillips, O.L., Baker, T., Costa, A.C.L., Espejo, J.S., Higuchi, N., Laurance, W.F., 2009. Influence of landscape heterogeneity on spatial patterns of wood productivity, wood specific density and above ground biomass in Amazonia. Biogeosciences 6, 1883-1902.

Anderson, L.O., Malhi, Y., Aragão, L.E., Ladle, R., Arai, E., Barbier, N., Phillips, O., 2010. Remote sensing detection of droughts in Amazonian forest canopies. New Phytologist 187, 733-750.

Anderson-Teixeira, K.J., Davies, S.J., Bennett, A.C., Gonzalez-Akre, E.B., MullerLandau, H.C., Joseph Wright, S., et al., 2015. CTFS-Forest GEO: a worldwide network monitoring forests in an era of global change. Global Change Biology 21, $528-549$.

Aragao, L.E., Poulter, B., Barlow, J.B., Anderson, L.O., Malhi, Y., Saatchi, S., Phillips, O. L., Gloor, E., 2014. Environmental change and the carbon balance of Amazonian forests. Biological Reviews 89, 913-931.

Avitabile, V., Herold, M., Heuvelink, G.B., Lewis, S.L., Phillips, O.L., Asner, G.P., Armston, J., Ashton, P.S., Banin, L., Bayol, N., et al., 2016. An integrated pantropical biomass map using multiple reference datasets. Global Change Biology 22, $1406-1420$.

Baker, T.R., Phillips, O.L., Malhi, Y., Almeida, S., Arroyo, L., Di Fiore, A., Erwin, T., Killeen, T.J., Laurance, S.G., Laurance, W.F., et al., 2004a. Variation in wood density determines spatial patterns in Amazonian forest biomass. Global Change Biology 10, 545-562.

Baker, T.R., Phillips, O.L., Malhi, Y., Almeida, S., Arroyo, L., Di Fiore, A., Erwin, T., Higuchi, N., Killeen, T.J., Laurance, S.G., Laurance, W.F., 2004b. Increasing biomass in Amazonian forest plots. Philosophical Transactions of the Royal Society of London. Series B: Biological Sciences 359, 353-365.

Baker, T.R., Phillips, O.L., Laurance, W.F., Pitman, N.C., Almeida, S., Arroyo, L., DiFiore, A., Erwin, T., et al., 2009. Do species traits determine patterns of wood production in Amazonian forests? Biogeosciences 6, 297-307.

Baker, T.R., Pennington, R.T., Dexter, K.G., Fine, P.V., Fortune-Hopkins, H., Honorio, E. N., Huamantupa-Chuquimaco, I., et al., 2017. Maximising synergy among tropical plant systematists, ecologists, and evolutionary biologists. Trends in Ecology \& Evolution 32, 258-267.

Baker, T.R., Vicuña Minano, E., Banda, K., del Castillo, D., Farfan-Rios, W, Lawson, I.T., Loja Alemán, E., Pallqui Camacho, N., Silman, M.R., Roucoux, K.H., Phillips, O.L., Honorio Coronado, E.N., Monteagudo Mendoza, Rojas Gonzáles, R., 2020. From plots to policy: how to ensure long-term forest plot data supports environmental management in intact tropical forest landscapes. People, Plants, Planet (in press).

Ballantyne, A.P., Alden, C.B., Miller, J.B., Tans, P.P., White, J.W.C., 2012. Increase in observed net carbon dioxide uptake by land and oceans during the past 50 years. Nature 488, 70-72.

Banin, L., Feldpausch, T.R., Phillips, O.L., Baker, T.R., Lloyd, J., Affum-Baffoe, K., Arets, E.J., Berry et al., 2012. What controls tropical forest architecture? Testing environmental, structural and floristic drivers. Global Ecology and Biogeography, $21,1179-1190$.

Banin, L., Lewis, S.L., Lopez-Gonzalez, G., Baker, T.R., Quesada, C.A., Chao, K.J., Burslem, D.F., Nilus, R., Abu Salim, K., Keeling, H.C., Tan, S., 2014. Tropical forest wood production: a cross-continental comparison. Journal of Ecology 102, 1025-1037.

Barlow, J., Lennox, G.D., Ferreira, J., Berenguer, E., Lees, A.C., Mac Nally, R. Thomson, J.R., de Barros Ferraz, S.F., Louzada, J., Oliveira, V.H.F., Parry, L., 2016. Anthropogenic disturbance in tropical forests can double biodiversity loss from deforestation. Nature 535, 144-147. 
Berry, N.J., Phillips, O.L., Ong, R.C., Hamer, K.C., 2008. Impacts of selective logging on tree diversity across a rainforest landscape: the importance of spatial scale. Landscape Ecology 23, 915-929.

Brearley, F.Q., Adinugroho, W.C., Cámara-Leret, R., Krisnawati, H., Ledo, A., Qie, L., Smith, T.E., Aini, F., Garnier, F., Lestari, N.S., Mansur, M., 2019. Opportunities and challenges for an Indonesian forest monitoring network. Annals of Forest Science 76, 54.

Brienen, R.J., Phillips, O.L., Feldpausch, T.R., Gloor, E., Baker, T.R., Lloyd, J., LopezGonzalez, G., Monteagudo-Mendoza, A., Malhi, Y., Lewis, S.L., Martinez, R.V., 2015 Long-term decline of the Amazon carbon sink. Nature 519, 344-348.

Bruelheide, H., Dengler, J., Jiménez-Alfaro, B., Purschke, O., Hennekens, S.M., Chytrý, M., Pillar, V.D., Jansen, F., Kattge, J., Sandel, B., Aubin, I., 2019. sPlot-A new tool for global vegetation analyses. Journal of Vegetation Science 30, 161-186.

Castillo, P.S.B., Alvarez, M., 2020. Forest Inventory and Analysis Fiscal Year 2018 Business Report. United States Department of Agriculture, p. 78.

Chave, J., Davies, S.J., Phillips, O.L., Lewis, S.L., Sist, P., Schepaschenko, D. Armiston, J., Baker, T.R., Coomes, D., et al., 2019. Ground data are essential for biomass remote sensing missions. Surveys in Geophysics 40, 863-880.

Chazdon, R.L., Broadbent, E.N., Rozendaal, D.M., Bongers, F., Zambrano, A.M.A., Aide, T.M., Balvanera, P., Becknell, J.M., Boukili, V., Brancalion, P.H., et al., 2016. Carbon sequestration potential of second-growth forest regeneration in the Latin American tropics. Science Advances 2 (5), e1501639.

Clinebell, R., Phillips, O.L., Gentry, A.H., Stark, N., Zuuring, H., 1995. Prediction of neotropical woody plant diversity from soil and climatic data. Biodiversity and Conservation 4, 56-90.

Coelho de Souza, F., Dexter, K.G., Phillips, O.L., Pennington, R.T., Neves, D., Sullivan, M. J., Alvarez-Davila, E., Alves, Á., Amaral, I., Andrade, A., Aragao, L.E., 2019. Evolutionary diversity is associated with wood productivity in Amazonian forests. Nature Ecology \& Evolution 3, 1754-1761.

Condit, R.S., 1997. Forest turnover, diversity, and $\mathrm{CO}_{2}$. Trends in Ecology \& Evolution 12, 249-250.

Corlett, R.T., Primack, R.B., 2011. Tropical Rain Forests: An Ecological and Biogeographical Comparison, 2nd edition. Wiley-Blackwell.

Davis, T.A.W., Richards, P.W., 1933. The vegetation of Moraballi Creek, British Guiana: an ecological study of a limited area of tropical rain forest. Part I. Journal of Ecology 21, 350-384.

Dawkins, H.C., Philip, M.S., 1998. Tropical moist forest silviculture and management: a history of success and failure. In: CAB International.

Di Marco, M., Chapman, S., Althor, G., Kearney, S., Besancon, C., Butt, N., Maina, J.M., Possingham, H.P., von Bieberstein, K.R., Venter, O., Watson, J.E., 2017. Changing trends and persisting biases in three decades of conservation science. Global Ecology and Conservation 10, 32-42.

Disney, M.I., Boni Vicari, M., Burt, A., Calders, K., Lewis, S.L., Raumonen, P., Wilkes, P., 2018. Weighing trees with lasers: advances, challenges and opportunities. Interface Focus 8 (2), 20170048.

Doughty, C.E., Metcalfe, D.B., Girardin, C.A.J., Amézquita, F.F., Cabrera, D.G., Huasco, W.H., Silva-Espejo, J.E., Araujo-Murakami, A., Da Costa, M.C., Rocha, W., Feldpausch, T.R., et al., 2015. Drought impact on forest carbon dynamics and fluxes in Amazonia. Nature 519, 78-82.

Drew, A.P., Boley, J.D., Zhao, Y., Wadsworth, F.H., 2009. Sixty-two years of change in subtropical wet forest structure and composition at El Verde. Puerto Rico. Interciencia 34, 34-40.

DRYFLOR, 2016. Plant diversity patterns and their conservation implications in neotropical dry forests. Science 353, 1383-1387.

Duncanson, L., Armston, J., Disney, M., Avitabile, V., Barbier, N., Calders, K., Carter, S., Chave, J., Herold, M., Crowther, T.W., Falkowski, M., et al., 2019. The importance of consistent global forest aboveground biomass product validation. Surveys in Geophysics 40, 979-999.

Elias, F., Ferreira, J., Lennox, G.D., Berenguer, E., Ferreira, S., Schwartz, G., Melo, L.D.O. Reis Junior, D.N., Nascimento, R.O., Ferreira, F.N., Espirito-Santo, F., 2020. Assessing the growth and climate sensitivity of secondary forests in highly deforested Amazonian landscapes. Ecology 101 (3), e02954.

Espírito-Santo, F.D., Gloor, M., Keller, M., Malhi, Y., Saatchi, S., Nelson, B., Junior, R.C., Pereira, C., Lloyd, J., et al., 2014. Size and frequency of natural forest disturbances and the Amazon forest carbon balance. Nature Communications 5, 3434.

Esquivel-Muelbert, A., Baker, T.R., Dexter, K.G., Lewis, S.L., ter Steege, H., LopezGonzalez, G., Monteagudo Mendoza, A., Brienen, R., Feldpausch, T.R., Pitman, N., Alonso, A., et al., 2017. Seasonal drought limits tree species across the Neotropics. Ecography 40, 618-629.

Esquivel-Muelbert, A., Baker, T.R., Dexter, K.G., Lewis, S.L., Brienen, R.J., Feldpausch, T. R., Lloyd, J., Monteagudo-Mendoza, A., Arroyo, L., Álvarez-Dávila, E., Higuchi, N., et al., 2019. Compositional response of Amazon forests to climate change. Global Change Biology 25, 39-56.

Fadrique, B., Báez, S., Duque, Á., Malizia, A., Blundo, C., Carilla, J., Osinaga-Acosta, O., Malizia, L., Silman, M., Farfán-Ríos, W., et al., 2018. Widespread but heterogeneous responses of Andean forests to climate change. Nature 564, 207-212.

Fauset, S., Baker, T.R., Lewis, S.L., Feldpausch, T.R., Affum-Baffoe, K., Foli, E.G. Hamer, K.C., Swaine, M.D., et al., 2012. Drought-induced shifts in the floristic and functional composition of tropical forests in Ghana. Ecology Letters 15, 1120-1129.

Fauset, S., Johnson, M.O., Gloor, M., Baker, T.R., Monteagudo, A., Brienen, R.J., Feldpausch, T.R., et al., 2015. Hyperdominance in Amazonian forest carbon cycling. Nature Communications 6, 6857.

Feldpausch, T.R., Banin, L., Phillips, O.L., Baker, T.R., Lewis, S.L., Quesada, C.A., AffumBaffoe, K., Arets, E.J., Berry, N.J., Bird, M., et al., 2011. Height-diameter allometry of tropical forest trees. Biogeosciences 8, 1081-1106.
Feldpausch, T.R., Lloyd, J., Lewis, S.L., Brienen, R.J., Gloor, M., Monteagudo Mendoza, A., Lopez-Gonzalez, G., Banin, L., et al., 2012. Tree height integrated into pantropical forest biomass estimates. Biogeosciences 9, 3381-3403.

Feldpausch, T.R., Phillips, O.L., Brienen, R.J.W., Gloor, E., Lloyd, J., Lopez-Gonzalez, G., Monteagudo-Mendoza, A., Malhi, Y., Alarcón, A., Dávila, E.Á., Alvarez-Loayza, P., 2016. Amazon forest response to repeated droughts. Global Biogeochemical Cycles 30, 964-982.

Ferreira, J., Lennox, G.D., et al., 2018. Carbon-focused conservation may fail to protect the most biodiverse tropical forests. Nature Climate Change. https://doi.org/ 10.1038/s41558-018-0225-7.

Forest Carbon Partnership Facility (FCPF) Carbon Fund, 2017. Emission Reductions Programme Document. Ghana Cocoa Forest REDD+ Programme (GCFRP). World Bank, ER-PD, p. 339.

Fyllas, N.M., Patiño, S., Baker, T.R., Bielefeld Nardoto, G., Martinelli, L.A., Quesada, C. A., Paiva, R., Schwarz, M., et al., 2009. Basin-wide variations in foliar properties of Amazonian forest: phylogeny. soils and climate. Biogeosciences 6, 2677-2708.

Gatti, L.V., Gloor, M., Miller, J.B., Doughty, C.E., Malhi, Y., Domingues, L.G., Basso, L.S., et al., 2014. Drought sensitivity of Amazonian carbon balance revealed by atmospheric measurements. Nature 506, 76-80.

Gaubert, B., Stephens, B.B., Basu, S., Chevallier, F., Deng, F., Kort, E.A., Patra, P.K., Peters, W., et al., 2019. Global atmospheric $\mathrm{CO}_{2}$ inverse models converging on neutral tropical land exchange, but disagreeing on fossil fuel and atmospheric growth rate. Biogeosciences 16, 117-134.

Gentry, A.H., 1988a. Tree species richness of upper Amazonian forests. Proceedings of the National Academy of Sciences 85, 156-159.

Gentry, A.H., 1988b. Changes in plant community diversity and floristic composition on environmental and geographical gradients. Annals of the Missouri Botanical Garden $75,1-34$.

Gentry, A.H., Vasquez, R., 1993. A Field Guide to the Families and Genera of Woody Plants of Northwest South America (Colombia, Ecuador, Peru): With Supplementary Notes on Herbaceous Taxa.

Gloor, M., Phillips, O.L., Lloyd, J.J., Lewis, S.L., Malhi, Y., Baker, T.R., LopezGonzalez, G., et al., 2009. Does the disturbance hypothesis explain the biomass increase in basin-wide Amazon forest plot data? Global Change Biology 15 2418-2430.

Gosline, G., Marshall, A.R., Larridon, I., 2019. Revision and new species of the African genus Mischogyne (Annonaceae). Kew Bulletin 74, 28.

Gourlet-Fleury, S., Mortier, F., Fayolle, A., Baya, F., Ouédraogo, D., Bénédet, F., Picard, N., 2013. Tropical forest recovery from logging: a 24 year silvicultural experiment from Central Africa. Philosophical Transactions of the Royal Society B: Biological Sciences 368 (1625), 20120302.

Harley, P., Vasconcellos, P., Vierling, L., Pinheiro, C.C.D.S., Greenberg, J., Guenther, A., Klinger, L., Almeida, S.S.D., Neill, D., Baker, T., Phillips, O., Malhi, Y., 2004. Variation in potential for isoprene emissions among Neotropical forest sites. Global Change Biology 10, 630-650.

Honorio Coronado, E.N., Baker, T.R., Phillips, O.L., Pitman, N.C., Pennington, R.T., Vásquez Martinez, R., Monteagudo, A., et al., 2009. Multi-scale comparisons of tree composition in Amazonian terra firme forests. Biogeosciences 30, 2719-2731.

Hubau, W., Lewis, S.L., Phillips, O.L., Affum-Baffoe, K., Beeckman, H., Cuní-Sanchez, A., Daniels, A.K., Ewango, C.E.N., et al., 2020. Asynchronous carbon sink saturation in the world's largest tropical forests. Nature 579, 80-87.

Hubbell, S.P., 1979. Tree dispersion, abundance, and diversity in a tropical dry forest. Science 203, 1299-1309.

Hubbell, S.P., Foster, R.B., 1983. Diversity of canopy trees in a neotropical forest and implications for the conservation of tropical trees. In: Sutton, S.J., Whitmore, T.C. Chadwick, A.C. (Eds.), Tropical Rainforest Ecology and Management. Blackwell, Oxford, pp. 25-41.

Huntingford, C., Zelazowski, P., Galbraith, D., Mercado, L.M., Sitch, S., Fisher, R., et al., 2013. Simulated resilience of tropical rainforests to CO2-induced climate change. Nature Geoscience 6, 268-273.

Johnson, M.O., Galbraith, D., Gloor, M., De Deurwaerder, H., Guimberteau, M., Rammig, A., Thonicke, K., et al., 2016. Variation in stem mortality rates determines patterns of above-ground biomass in Amazonian forests: implications for dynamic global vegetation models. Global Change Biology 22, 3996-4013.

Joint Research Centre, 2003. Global Land Cover 2000 Database. European Commission. In: Joint Research Centre. https://forobs.jrc.ec.europa.eu/products/glc2000/data _access.php.

Kohyama, T.S., Kohyama, T.I., Sheil, D., 2019. Estimating net biomass production and loss from repeated measurements of trees in forests and woodlands: Formulae, biases and recommendations. Forest Ecology and Management 433, 729-740.

Lawrence, A., Phillips, O.L., Ismodes, A.R., Lopez, M., Rose, S., Wood, D., Farfan, A.J., 2005. Local values for harvested forest plants in Madre de Dios, Peru: towards a more contextualised interpretation of quantitative ethnobotanical data. Biodiversity \& Conservation 14, 45-79.

Levis, C., Costa, F.R., Bongers, F., Peña-Claros, M., Clement, C.R., Junqueira, A.B., Neves, E.G., Tamanaha, E.K., Figueiredo, F.O., Salomão, R.P., Castilho, C.V., 2017. Persistent effects of pre-Columbian plant domestication on Amazonian forest composition. Science 355, 925-931.

Lewis, S.L., Brando, P.M., Phillips, O.L., van der Heijden, G.M., Nepstad, D., et al., 2011. The 2010 amazon drought. Science 331-554.

Lewis, S.L., Malhi, Y., Phillips, O.L., 2004. Fingerprinting the impacts of global change on tropical forests. Philosophical Transactions of the Royal Society of London. Series B: Biological Sciences 359, 437-462.

Lewis, S.L., Phillips, O.L., Sheil, D., Vinceti, B., Baker, T.R., Brown, S., Graham, A.W., Higuchi, N., Hilbert, D.W., et al., 2004a. Tropical forest tree mortality, recruitment 
and turnover rates: calculation, interpretation and comparison when census intervals vary. Journal of Ecology 92, 929-944.

Lewis, S.L., Phillips, O.L., Baker, T.R., Lloyd, J., Malhi, Y., Almeida, S., Higuchi, N., Laurance, W.F., Neill, D.A., Silva, J.N.M., Terborgh, J., 2004b. Concerted changes in tropical forest structure and dynamics: evidence from 50 South American long-term plots. Philosophical Transactions of the Royal Society of London. Series B: Biological Sciences 359, 421-436.

Lewis, S.L., Lopez-Gonzalez, G., Sonké, B., Affum-Baffoe, K., Baker, T.R., Ojo, L.O., Phillips, O.L., Reitsma, J.M., White, L., Comiskey, J.A., Ewango, C.E., et al., 2009. Increasing carbon storage in intact African tropical forests. Nature 457, 1003-1006.

Lewis, S.L., Sonké, B., Sunderland, T., Begne, S.K., Lopez-Gonzalez, G., van der Heijden, G.M.F., Phillips, O.L., et al., 2013. Above-ground biomass and structure of 260 African tropical forests. Philosophical Transactions of the Royal Society B: Biological Sciences 368, 20120295.

López-González, G., Phillips, O.L., 2012. Estudiando el Amazonas: la experiencia de la Red Amazónica de Inventarios Forestales. Revista Ecosistemas 21, 1-2.

López-Gonzalez, G., Lewis, S.L., Burkitt, M., Phillips, O.L., 2011. ForestPlots.net: a web application and research tool to manage and analyse tropical forest plot data. Journal of Vegetation Science 22, 610-613.

López-Gonzalez, G., Sullivan, M.J.P., Baker, T.R., 2015. BiomasaFP: Tools for analysing data downloaded from ForestPlots.net. R package version 1, 1.

Malhi, Y., Phillips, O.L., Lloyd, J., Baker, T., Wright, J., Almeida, S., Arroyo, L., Frederiksen, T., Grace, J., Higuchi, N., et al., 2002. An international network to monitor the structure, composition and dynamics of Amazonian forests (RAINFOR). Journal of Vegetation Science 13, 439-450.

Malhi, Y., Baker, T.R., Phillips, O.L., Almeida, S., Alvarez, E., Arroyo, L., Chave, J., Czimczik, C.I., Fiore, A.D., Higuchi, N., Killeen, T.J., 2004. The above-ground coarse wood productivity of 104 Neotropical forest plots. Global Change Biology 10, 563-591.

Malhi, Y., Wood, D., Baker, T.R., Wright, J., Phillips, O.L., Cochrane, T., Meir, P., Chave, J., Almeida, S., Arroyo, L., et al., 2006. The regional variation of aboveground live biomass in old-growth Amazonian forests. Global Change Biology $12,1107-1138$.

Malhi, Y., Farfán Amézquita, F., Doughty, C.E., Silva-Espejo, J.E., Girardin, C.A., Metcalfe, D.B., Aragão, L.E., Huaraca-Quispe, L.P., et al., 2014a. The productivity, metabolism and carbon cycle of two lowland tropical forest plots in south-western Amazonia, Peru. Plant Ecology \& Diversity 7, 85-105.

Malhi, Y., Gardner, T.A., Goldsmith, G.R., Silman, M.R., Zelazowski, P., 2014b. Tropical forests in the Anthropocene. Annual Review of Environment and Resources 39.

Malhi, Y., Girardin, C., Metcalfe, D., Doughty, C., Aragão, L.E.O., Rifai, S., Shenkin, A., Aguirre Gutierrez, J., Dahlsjö, C., et al., 2021. The Global Ecosystems Monitoring network: monitoring ecosystem productivity and carbon cycling across the tropics. Biological Conservation. Biological Conservation 253, 108889.

Malizia, A., Blundo, C., Carilla, J., Osinaga-Acosta, O., Cuesta, F., Duque, A., Aguirre, N., Aguirre, Z., Ataroff, M., Baez, S., et al., 2020. Elevation and latitude drives structure and tree species composition in Andean forests: results from a large-scale plot Network. PLoS ONE 15, e0231553.

Marimon, B.S., Marimon-Junior, B.H., Feldpausch, T.R., Oliveira-Santos, C., Mews, H.A., et al., 2014. Disequilibrium and hyperdynamic tree turnover at the forest-cerrado transition zone in southern Amazonia. Plant Ecology \& Diversity 7, 281-292.

McDowell, N., Allen, C.D., Anderson-Teixeira, K., Brando, P., Brienen, R., Chambers, J. Christoffersen, B., Davies, S., Doughty, C., Duque, A., Espirito-Santo, F., 2018. Drivers and mechanisms of tree mortality in moist tropical forests. New Phytologist 219, 851-869.

Mitchard, E.T.A., Feldpausch, T.R., Brienen, R.J.W., Lopez-Gonzalez, G., Monteagudo, A., Baker, T.R., et al., 2014. Markedly divergent estimates of Amazon forest carbon density from ground plots and satellites. Global Ecology and Biogeography. https://doi.org/10.1111/geb.12168.

Monteagudo Mendoza, A., Vásquez Martínez, R., Rojas Gonzales, R., Phillips, O.L., Baker, T.R., Dueñas Linares, H., Pickavance, G.C., Núñez Vargas, P., et al., 2020 Primer Catálogo de los Arboles de la Amazonía de Madre De Dios, Perú. Universidad Andina del Cusco, Cusco, Peru, p. 240.

Nakamura, A., Kitching, R.L., Cao, M., Creedy, T.J., Fayle, T.M., Freiberg, M., Hewitt, C. N., Itioka, T., et al., 2017. Forests and their canopies: achievements and horizons in canopy science. Trends in Ecology \& Evolution 32, 438-451.

Nogueira, D.S., Marimon, B.S., Marimon-Junior, B.H., Oliveira, E.A., Morandi, P., Reis, S. M., Elias, F., Neves, E.C., Feldpausch, T.R., Lloyd, J., Phillips, O.L., 2019. Impacts of fire on forest biomass dynamics at the southern Amazon edge. Environmental Conservation 46, 285-292.

Norden, N., González-M, R., Avella-M, A., Salgado-Negret, B., Alcázar, C., RodríguezBuriticá, S., Aguilar-Cano, J., et al., 2020, Building a socio-ecological monitoring platform for the comprehensive management of tropical dry forests. Plants, People, Planet. https://doi.org/10.1002/ppp3.10113.

Oliveira, E.A., Marimon-Junior, B.H., Iriarte, J., Morandi, P.S., Maezumi, S.Y., Nogueira, D.S., Aragão, L.E.O.C., Feldpausch, T.R., 2020. Legacy of Amazonia Dark Earth soil on forest structure and species composition. Global Ecology and Biogeography 29, 1458-1473.

Olson, D.M., Dinerstein, E., Wikramanayake, E.D., Burgess, N.D., Powell, G.V., Underwood, E.C., D'Amico, J.A., et al., 2001. Terrestrial ecoregions of the world: a new map of life on Earth. Bioscience 51, 93-938.

Pallqui, N.C., Monteagudo, A., Phillips, O.L., Lopez-Gonzalez, G., Cruz, L., Galiano, W., Chavez, W., Vasquez, R., 2014. Dinámica, biomasa aérea y composición florística en parcelas permanentes Reserva Nacional Tambopata, Madre de Dios, Perú. Revista Peruana de Biología 21, 235-242.
Pan, Y., Birdsey, R.A., Fang, J., Houghton, R., Kauppi, P.E., et al., 2011. A large and persistent carbon sink in the world's forests. Science 333, 988-993. https://doi. org/10.1126/science.1201609.

Patiño, S., Lloyd, J., Paiva, R., Baker, T.R., Quesada, C.A., Mercado, L.M., et al., 2009. Branch xylem density variations across the Amazon Basin. Biogeosciences 6, 545-568.

Peacock, J., Baker, T.R., Lewis, S.L., Lopez-Gonzalez, G., Phillips, O.L., 2007. The RAINFOR database: monitoring forest biomass and dynamics. Journal of Vegetation Science 18, 535-542.

Pennington, T., Lehmann, C.E., Rowland, L., 2018. Tropical savannas and dry forests. Current Biology 28, 541-545.

Peres, C.A., Emilio, T., Schietti, J., Desmoulière, S.J., Levi, T., 2016. Dispersal limitation induces long-term biomass collapse in overhunted Amazonian forests. Proceedings of the National Academy of Sciences 113, 892-897.

Phillips, O.L., 1996. Long-term environmental change in tropical forests: increasing tree turnover. Environmental Conservation 23, 235-248.

Phillips, O.L., Brienen, R.J., 2017. Carbon uptake by mature Amazon forests has mitigated Amazon nations' carbon emissions. Carbon Balance and Management 12, 1.

Phillips, O., Gentry, A.H., 1993. The useful plants of Tambopata, Peru: I. Statistical hypotheses tests with a new quantitative technique. Economic Botany 47, 15-32.

Phillips, O.L., Gentry, A.H., 1994. Increasing turnover through time in tropical forests. Science 263, 954-958.

Phillips, O., Miller, J.S., 2002. Global patterns of plant diversity: Alwyn H. Gentry's forest transect data set. In: Monographs in Systematic Botany, 89. Missouri Botanical Press, pp. 1-319.

Phillips, O.L., Raven, P.H., 1997. A strategy for sampling neotropical forests. Neotropical Biodiversity and Conservation 1, 141-165.

Phillips, O., Sheil, D., 1997. Forest turnover. diversity and $\mathrm{CO}_{2}$. Trends in Ecology \& Evolution 12, 404.

Phillips, O.L., Hall, P., Gentry, A.H., Sawyer, S.A., Vasquez, R., 1994. Dynamics and species richness of tropical rain forests. Proceedings of the National Academy of Sciences of the United States of America 91, 2805-2809.

Phillips, O.L., Malhi, Y., Higuchi, N., Laurance, W.F., Núnez, P.V., Vásquez, R.M., Laurance, S.G., Ferreira, L.V., Stern, M., Brown, S., Grace, J., 1998. Changes in the carbon balance of tropical forests: evidence from long-term plots. Science 282 , 439-442.

Phillips, O.L., Baker, T., Feldspauch, T., \& Brienen, R. J. W., 2002. Field manual for plot establishment and remeasurement (RAINFOR). Amazon Forest Inventory Network, Sixth Framework Programme (2002-2006). URL: http://www.geog.leeds.ac. uk/projects/rainfor.

Phillips, O.L., Baker, T.R., Arroyo, L., Higuchi, N., Killeen, T.J., Laurance, W.F., Lewis, S. L., Lloyd, J., Malhi, Y., Monteagudo, A., Neill, D.A., 2004. Pattern and process in Amazon tree turnover, 1976-2001. Philosophical Transactions of the Royal Society of London. Series B: Biological Sciences 359, 381-407.

Phillips, O.L., Lewis, S.L., Baker, T.R., Chao, K.J., Higuchi, N., 2008. The changing Amazon forest. Philosophical Transactions of the Royal Society B: Biological Sciences 363, 1819-1827.

Phillips, O.L., Aragão, L.E., Lewis, S.L., Fisher, J.B., Lloyd, J., López-González, G., Malhi, Y., Monteagudo, A., Peacock, J., Quesada, C.A., Van Der Heijden, G., 2009. Drought sensitivity of the Amazon rainforest. Science 323, 1344-1347.

Phillips, O.L., Sullivan, M.J., Baker, T.R., Mendoza, A.M., Vargas, P.N., Vásquez, R., 2019. Species matter: wood density influences tropical forest biomass at multiple scales. Surveys in Geophysics 404, 913-935.

Phillips, O.L., Vásquez Martínez, R., Monteagudo Mendoza, A., Baker, T.R., Núñez Vargas, P., et al., 2005. Large lianas as hyperdynamic elements of the tropical forest canopy. Ecology 86, 1250-1258.

Piao, S., Wang, X., Park, T., Chen, C., Lian, X., He, Y., Bjerke, J.W., Chen, A., Ciais, P., Tømmervik, H., Nemani, R.R., 2019. Characteristics, drivers and feedbacks of global greening. Nature Reviews Earth \& Environment 1, 14.

Pires, J.M., Salomão, R.P., 2000. Dinâmica da diversidade arbórea de um fragmento de floresta tropical primária na Amazônia oriental - 1. Período: 1956 a 1992. Bol. Mus. Para. Emílio goeldi, Sér. Botânica, 16, pp. 63-110.

Pomeroy, M., Primack, R.B., Rai, S.N., 2003. Changes in four rain forest plots of the Western Ghats, India, 1939-93. Conservation and Society 1, 113-135.

Poulsen, J.R., Medjibe, V.P., White, L.J., Miao, Z., Banak-Ngok, L., Beirne, C., Clark, C.J., Cuni-Sanchez, A., et al., 2020. Old growth Afrotropical forests critical for maintaining forest carbon. Global Ecology and Biogeography 29, 1785-1798.

Qie, L., Lewis, S.L., Sullivan, M.J., Lopez-Gonzalez, G., Pickavance, G.C., Sunderland, T., Ashton, P., Hubau, W., et al., 2017. Long-term carbon sink in Borneo's forests halted by drought and vulnerable to edge effects. Nature Communications 8, 1966.

Quesada, C.A., Phillips, O.L., Schwarz, M., Czimczik, C.I., Baker, T.R., Patiño, S., Fyllas, N.M., Hodnett, M.G., Herrera, R., et al., 2012. Basin-wide variations in Amazon forest structure and function are mediated by both soils and climate. Biogeosciences 9, 2203-2246. https://doi.org/10.5194/bg-9-2203-2012.

Reboredo Segovia, A.L., Romano, D., Armsworth, P.R., 2020. Who studies where? Boosting tropical conservation research where it is most needed. Frontiers in Ecology and the Environment 18, 159-166.

Reis, S.M., Marimon, B.S., Marimon-Junior, B.H., Morandi, P.S., Oliveira, E.A., Eias, F., Neves, E.C., Oliveira, B., Nogueira, D.S., Umetsu, R.K., Feldpausch, T.R., Phillips, O. L., 2018. Climate and fragmentation affect forest structure at the Southern border of Amazonia. Plant Ecology \& Diversity 11, 13-25.

Reitsma, J.M., 1988. Forest Vegetation of Gabon. TROPENBOS Technical Series. In: ISBN: 90-5113-007-4.

Requena Suarez, D., Rozendaal, D.M., De Sy, V., Phillips, O.L., Alvarez-Dávila, E., Anderson-Teixeira, K., Araujo-Murakami, A., Arroyo, L., Baker, T.R., Bongers, F., 
et al., 2019. Estimating aboveground net biomass change for tropical and subtropical forests: Refinement of IPCC default rates using forest plot data. Global Change Biology 25, 3609-3624.

Ríos-Saldaña, C.A., Delibes-Mateos, M., Ferreira, C.C., 2018. Are fieldwork studies being relegated to second place in conservation science? Global Ecology and Conservation 14, e00389.

Rowland, L., da Costa, A.C.L., Galbraith, D.R., Oliveira, R.S., Binks, O.J., Oliveira, A.A.R., Pullen, A.M., Doughty, C.E., Metcalfe, D.B., Vasconcelos, S.S., Ferreira, L.V., 2015. Death from drought in tropical forests is triggered by hydraulics not carbon starvation. Nature 528, 119-122.

Salomão, R.D., 1991. Uso de parceles permanentes para estudos da vegetaçao da floresta tropical úmida. I. Municıpio de Marabá, Pará. Boletim do Museu Paraense Emılio Goeldi. Serie Botânica 7, 543-604.

Schepaschenko, D., Chave, J., Phillips, O.L., et al., 2019. The Forest Observation System, building a global reference dataset for remote sensing of forest biomass. Sci Data 198 (2019), 6. https://doi.org/10.1038/s41597-019-0196-1.

Schietti, J., Martins, D., Emilio, T., Souza, P.F., Levis, C., Baccaro, F.B., et al., 2016. Forest structure along a $600 \mathrm{~km}$ transect of natural disturbances and seasonality gradients in central-southern Amazonia. Journal of Ecology 104, 1335-1346.

SEOSAW partnership, 2020. A network to understand the changing socio-ecology of the southern African woodlands (SEOSAW): Challenges, benefits, and methods. Plants, People, Planet 1-19. https://doi.org/10.1002/ppp3.10168.

Sheil, D., 1995. Evaluating turnover in tropical forests. Science 268, 894-896.

Sheil, D., 1996. Species richness, tropical forest dynamics and sampling: questioning cause and effect. Oikos 76, 587-590.

Sist, P., Rutishauser, E., Peña-Claros, M., Shenkin, A., Hérault, B., Blanc, L., Baraloto, C. Baya, F., Benedet, F., da Silva, K.E., Descroix, L., et al., 2015. The Tropical managed Forests Observatory: a research network addressing the future of tropical logged forests. Applied Vegetation Science 18, 171-174.

Sousa, T.R., Schietti, J., Coelho de Souza, F., Esquivel-Muelbert, A., Ribeiro, I.O., Emílio, T., et al., 2020. Palms and trees resist extreme drought in Amazon forests with shallow water tables. Journal of Ecology 108, 2070-2082.

Steidinger, B.S., Crowther, T.W., Liang, J., Van Nuland, M.E., Werner, G.D., Reich, P.B., Nabuurs, G.J., et al., 2019. Climatic controls of decomposition drive the global biogeography of forest-tree symbioses. Nature 569, 404-408.

Sullivan, M.J., Talbot, J., Lewis, S.L., Phillips, O.L., Qie, L., Begne, S.K., Chave, J., CuniSanchez, A., Hubau, W., Lopez-Gonzalez, G., Miles, L., 2017. Diversity and carbon storage across the tropical forest biome. Scientific Reports 7, 1-12.

Sullivan, M.J., Lewis, S.L., Hubau, W., Qie, L., Baker, T.R., Banin, L.F., Chave, J., CuniSanchez, A., Feldpausch, T.R., et al., 2018. Field methods for sampling tree height for tropical forest biomass estimation. Methods in Ecology and Evolution 9, 1179-1189.

Sullivan, M.J.P., Lewis, S.L., Affum-Baffoe, K., Castilho, C., Costa, F., Cuni Sanchez, A., Ewango, C.E.N., Hubau, W., Marimon, B., Monteagudo-Mendoza, A., et al., 2020.
Long-term thermal sensitivity of Earth's tropical forests. Science 368, 869-874. https://doi.org/10.1126/science.aaw7578.

Taylor, J.A., Lloyd, J., 1992. Sources and sinks of atmospheric CO2. Australian Journal of Botany 40, 407-418.

ter Steege, H., Pitman, N., Sabatier, D., Castellanos, H., Van Der Hout, P., Daly, D.C., Silveira, M., et al., 2003. A spatial model of tree $\alpha$-diversity and tree density for the Amazon. Biodiversity \& Conservation 12, 2255-2277.

ter Steege, H., Pitman, N.C., Phillips, O.L., Chave, J., Sabatier, D., Duque, A., Molino, J. F., Prévost, M.F., Spichiger, R., Castellanos, H., Von Hildebrand, P., 2006. Continental-scale patterns of canopy tree composition and function across Amazonia. Nature 443, 444-447.

ter Steege, H., Pitman, N.C., Sabatier, D., Baraloto, C., Salomão, R.P., Guevara, J.E., Phillips, O.L., Castilho, C.V., et al., 2013. Hyperdominance in the Amazonian tree flora. Science 342, 1243092.

Terrer, C., et al., 2019. Nitrogen and phosphorus constrain the CO2 fertilization of global plant biomass. Nature Climate Change 9, 684-689.

Vargas, R., Alcaraz-Segura, D., Birdsey, R., Brunsell, N.A., Cruz-Gaistardo, C.O., de Jong, B., Etchevers, J., Guevara, M., Hayes, D.J., Johnson, K., Loescher, H.W., 2017. Enhancing interoperability to facilitate implementation of REDD+: case study of Mexico. Carbon Management 8, 57-65.

Vásquez, M.R., Soto Shareva, Y., 2020. Virola pseudosebifera (Myristicaceae), una nueva especie para la Selva Alta del Perú, 10. Revista Q'ueña, pp. 7-12.

Vásquez, R., Rojas, R., Monteagudo, A.M., Valenzuela, L.G., Huamantupa, I., 2018. Catalogo de los Arboles del Perú. Q'ueña Revista de la Sociedad Botánica del Cusco 9 (1). In: número especial.

Vicuña Miñano, E., Baker, T.R., Banda, K., Coronado, E.H., Monteagudo, A., Phillips, O. L., Torres, D.D.C., Rios, W.F., Flores, G., Huaman, D., Huaman, K.T., et al., 2018. El sumidero de carbono en los bosques primarios Amazónicos es una oportunidad para lograr la sostenibilidad de su conservación. Folia Amazónica 27, 101-109.

Vilanova, E., Ramírez-Angulo, H., Torres-Lezama, A., Aymard, G., Gámez, L., Durán, C., Hernández, L., Herrera, R., van der Heijden, G., Phillips, O.L., Ettl, G.J., 2018. Environmental drivers of forest structure and stem turnover across Venezuelan tropical forests. PloS One 13 (6).

Villela, D.M., Nascimento, M.T., de Aragao, L.E.O., Da Gama, D.M., 2006. Effect of selective logging on forest structure and nutrient cycling in a seasonally dry Brazilian Atlantic forest. Journal of Biogeography 33, 506-516.

Werger, M.J.A. (Ed.), 2011. Sustainable Management of Tropical Rainforests: The CELOS Management System. Tropenbos International. Paramaribo, Suriname.

Wright, S.J., 2020. The Smithsonian Tropical Research Institute: ecological and applied research. Biological Conservation 252, 108858.

Wurdack, K.J., Farfan-Rios, W., 2017. Incadendron: a new genus of Euphorbiaceae tribe Hippomaneae from the sub-Andean cordilleras of Ecuador and Peru. PhytoKeys 85, $69-86$. 